Ann. Scient. Éc. Norm. Sup.,

$4^{\text {e }}$ série, t. 38, 2005, p. 609 à 669.

\title{
KLOOSTERMAN IDENTITIES OVER A QUADRATIC EXTENSION II
}

\author{
BY HERVÉ JACQUET ${ }^{1}$
}

To the memory of Yasuko Jacquet

ABSTRACT. - We prove certain identities between Kloosterman integrals. They constitute the fundamental lemma of a relative trace formula for Hecke functions. The main application of the trace formula in question is the following result. Let $E / F$ be a quadratic extension of number fields. A cuspidal automorphic representation of $G L\left(n, E_{\mathbb{A}}\right)$ is distinguished by some unitary group if and only if it is the base change of an automorphic cuspidal representation of $G L\left(n, F_{\mathbb{A}}\right)$.

(c) 2005 Elsevier SAS

RÉSUMÉ. - Nous prouvons certaines identités entre intégrales de Kloosterman. Elles constituent le lemme fondamental d'une formule des traces relatives pour les fonctions de Hecke générales. L'application principale de cette formule des traces est le résultat suivant. Soit $E / F$ une extension quadratique de corps de nombres. Une représentation automorphe cuspidale de $G L\left(n, E_{\mathbb{A}}\right)$ est distinguée par un groupe unitaire si et seulement si c'est le changement de base d'une représentation automorphe cuspidale de $G L\left(n, F_{\mathbb{A}}\right)$.

(c) 2005 Elsevier SAS

\section{Contents}

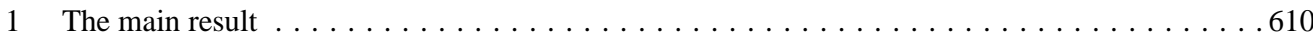

2 General matching . . . . . . . . . . . . . . . . . . . . . . . . . . . 6614

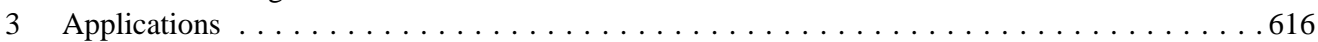

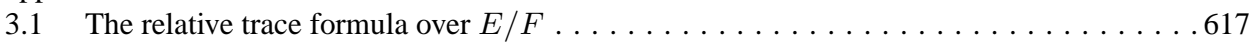

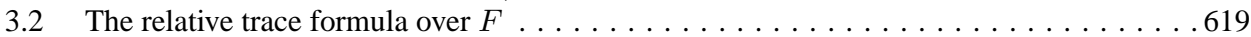

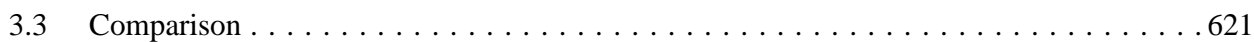

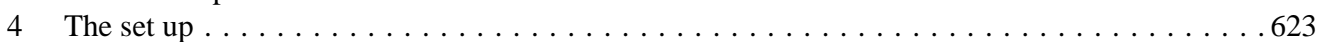

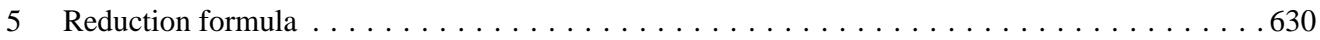

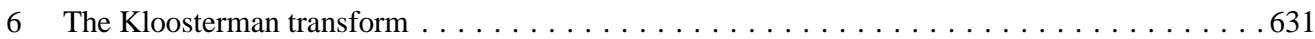

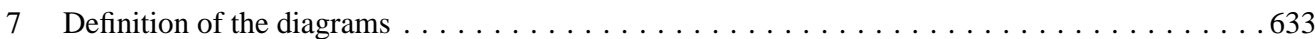

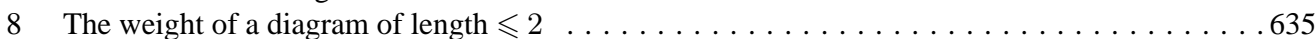

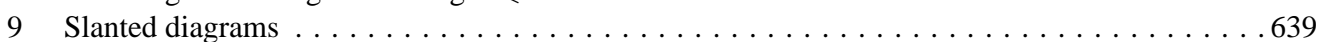

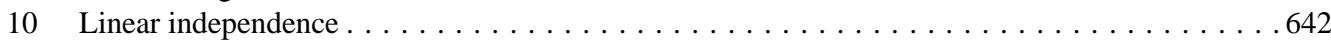

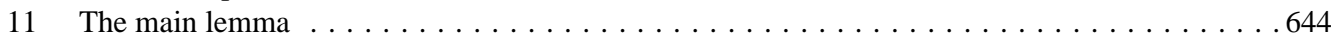

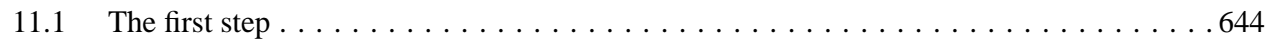

\footnotetext{
${ }^{1}$ Partially supported by NSF grant DMS-02-45310.

ANNALES SCIENTIFIQUES DE L'ÉCOLE NORMALE SUPÉRIEURE 0012-9593/04/C 2005 Elsevier SAS. All rights reserved
} 


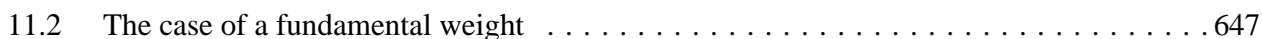

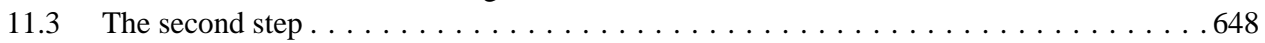

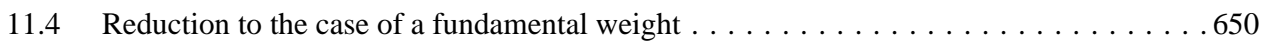

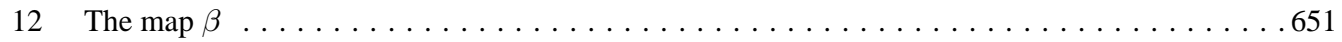

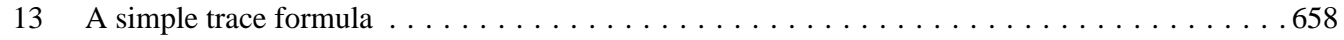

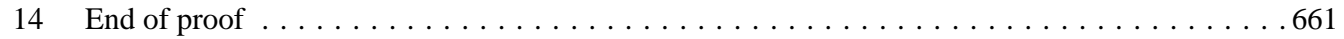

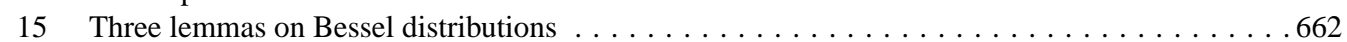

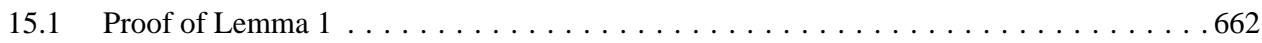

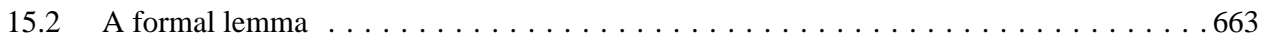

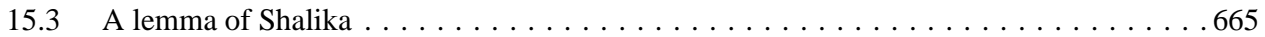

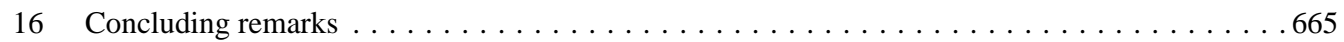

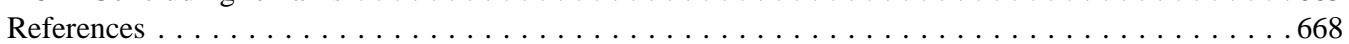

\section{The main result}

Let $F$ be a non-Archimedean local field of odd residual characteristic and characteristic 0 . We denote by $\varpi$ a uniformizer, by $q$ the cardinality of the residual field $\kappa$ and by $|x|_{F}$ or simply $|x|$ the absolute value of an element $x$. We consider the quadratic unramified extension $E / F$. Thus $\varpi$ is also a uniformizer for $E$. We let $\eta_{E / F}$ or simply $\eta$ be the corresponding unramified quadratic character of $F^{\times}$. When necessary we use the notation $|x|_{F}$ and $|z|_{E}$ to denote the absolute value on $F$ and $E$ respectively. However we adopt the following convention: when we write an inequality (or an equality) such as $|z| \leqslant\left|\varpi^{m}\right|$ the same absolute value is used on the two sides of the inequality: thus the inequality stands for either $|z|_{E} \leqslant\left|\varpi^{m}\right|_{E}$ or $|z|_{F} \leqslant\left|\varpi^{m}\right|_{F}$. We denote by $\sigma$ the Galois conjugation of $E / F$. We also write $\sigma(z)=\bar{z}$. Thus $|z|_{E}=|z \bar{z}|_{F}$. We let $\psi_{F}$ or simply $\psi$ be an additive character of $F$ whose conductor is the ring of integers $\mathcal{O}_{F}$ of $F$. Then $\psi_{E}(z):=\psi_{F}(z+\bar{z})$ is an additive character of $E$ whose conductor is $\mathcal{O}_{E}$.

We denote by $G_{n}$ or simply $G$ the group $G L(n)$. We let $N_{n}$ or simply $N$ be the subgroup of upper triangular matrices with unit diagonal and $A_{n}$ or simply $A$ the subgroup of diagonal matrices. We define a character

$$
\theta: N_{n}(F) \rightarrow \mathbb{C}^{\times}
$$

by

$$
\theta(u)=\psi\left(\sum_{1 \leqslant i \leqslant n-1} u_{i, i+1}\right) .
$$

Let $\mathcal{S}(M(n \times n, F))$ be the space of locally constant compactly supported functions on $M(n \times n, F)$. We define the (diagonal) orbital integral of a function $\Phi \in \mathcal{S}(M(n \times n, F))$. It is the function on $A_{n}(F)$ defined by

$$
\Omega[\Phi, \psi: a]:=\int \Phi\left({ }^{t} u_{1} a u_{2}\right) \theta\left(u_{1} u_{2}\right) d u_{1} d u_{2} .
$$

The integral is over $N_{n}(F) \times N_{n}(F)$ and the Haar measure $d u$ is normalized by the condition

$$
\int_{N_{n}\left(\mathcal{O}_{F}\right)} d u=1 .
$$

In fact, the orbital integral is defined on the set of diagonal matrices $a \in M(n \times n, F)$, with $a_{1} a_{2} \cdots a_{n-1} \neq 0$. We let $\Phi_{0}$ be the characteristic function of $M\left(n \times n, \mathcal{O}_{F}\right)$. We remark that if 
we define $\Phi^{t}$ by $\Phi^{t}(X)=\Phi\left({ }^{t} X\right)$ then

$$
\Omega[\Phi, \psi: a]=\Omega\left[{ }^{t} \Phi, \psi: a\right] .
$$

Similarly, we let $M_{h}(E / F, n)$ be the space of $n \times n$ Hermitian matrices, that is, the matrices $x \in M(n \times n, E)$ such that ${ }^{t} x=\bar{x}$. We let $\Psi_{0}$ be the characteristic function of

$$
M_{h}(E / F, n) \cap M\left(n \times n, \mathcal{O}_{E}\right) .
$$

We define a character

$$
u \mapsto \theta(u \bar{u})
$$

of $N_{n}(F)$ by

$$
\theta(u \bar{u})=\psi\left(\sum_{1 \leqslant i \leqslant n-1} u_{i, i+1}+\bar{u}_{i, i+1}\right) .
$$

The notation is justified by the fact that $u \bar{u}$ is the product of an element of $N(F)$ and an element of the derived group of $N(E)$.

If $\Psi$ is in $\mathcal{S}\left(M_{h}(E / F, n)\right)$ we define its (diagonal) orbital integral. It is the function on $A_{n}(F)$ defined by

$$
\Omega[\Psi, E / F, \psi: a]:=\int \Psi\left({ }^{t} \bar{u} a u\right) \theta(u \bar{u}) d u .
$$

The integral is over $N_{n}(E)$ and the Haar measure $d u$ is normalized by the condition

$$
\int_{N_{n}\left(\mathcal{O}_{E}\right)} d u=1
$$

Again the integral is in fact defined on the set of diagonal matrices $a \in M(n \times n, F)$ such that $a_{1} a_{2} \cdots a_{n-1} \neq 0$. Again

$$
\Omega\left[{ }^{t} \Psi, E / F, \psi: a\right]=\Omega[\Psi, E / F, \psi: a] .
$$

We say that $\Psi$ and $\Phi$ match and we write $\Psi \leftrightarrow \Phi$ if

$$
\Omega[\Psi, E / F, \psi: a]=\gamma(a) \Omega[\Phi, \psi: a]
$$

where $\gamma$ is the transfer factor defined by

$$
\gamma(a)=: \eta\left(a_{1}\right) \eta\left(a_{1} a_{2}\right) \cdots \eta\left(a_{1} a_{2} \cdots a_{n-1}\right) .
$$

We set

$$
K_{F}:=G L\left(n, \mathcal{O}_{F}\right), \quad K_{E}:=G L\left(n, \mathcal{O}_{E}\right),
$$

we denote by $\mathcal{H}(G L(n, F))$ or simply $\mathcal{H}_{F}$ the Hecke algebra, that is, the convolution algebra of bi- $K_{F}$-invariant functions of compact support on $G L(n, E)$. We define similarly $\mathcal{H}(G L(n, E))=\mathcal{H}_{E}$. 
We recall the base change homomorphism

$$
b: \mathcal{H}(G L(n, E)) \rightarrow \mathcal{H}(G L(n, F))
$$

of the Hecke algebras. If $\mathcal{S}(f)$ denotes the Satake transform of $f$ then

$$
\mathcal{S}(b(f))(x)=\mathcal{S}(f)\left(x^{2}\right) .
$$

Equivalently, if $\pi$ is an unramified representation of $G(F)$ corresponding to the character $f \mapsto \mathcal{S}(f)(x)$, we write $\mathcal{S}(f)(\pi)$ for $\mathcal{S}(f)(x)$. Then if $\Pi$ is the representation base change of $\pi$ we have

$$
\mathcal{S}(f)(\Pi)=\mathcal{S}(b(f))(\pi) .
$$

Our main result is as follows (fundamental lemma for Hecke functions).

THEOREM 1. - For any $f \in \mathcal{H}(G L(n, E))$ the function

$$
X \mapsto \int_{G L(n, E)} \Psi_{0}\left({ }^{t} \bar{g} X g\right) f(g) d g
$$

matches the function

$$
X \mapsto \int_{G L(n, F)} \Phi_{0}(X g) b(f)(g) d g .
$$

We remark that the same result is true in the case of positive characteristic. This can be derived from the work of $\mathrm{Ngô}$ [15]. The proof we give should extend to the case of characteristic, provided the necessary modifications be made in the global theory.

Of course the Haar measures are normalized by the condition that the measures of $K_{E}$ and $K_{F}$ are 1. If $f$ is the unit element of the Hecke algebra this result was proved in [6, (8)].

We remark that the second function has the same orbital integral as the function

$$
X \mapsto \int_{G L(n, F)} \Phi_{0}(g X) b(f)(g) d g .
$$

For any function $f$ on a group we define the function $\check{f}$ by $\check{f}(g)=f\left(g^{-1}\right)$. We first note that $b(\check{f})=b(f)$. Thus, at the cost of replacing the function $f$ by the function $\breve{f}$ we may formulate the result as the matching of the functions

$$
X \mapsto \int_{G L(n, E)} \Psi_{0}\left({ }^{t} \bar{g} X g\right) f\left(g^{-1}\right) d g
$$

and

$$
X \mapsto \int_{G L(n, F)} \Phi_{0}(X g) b(f)\left(g^{-1}\right) d g .
$$

Now let us apply the theorem to a function $f$ supported on the set $\left\{g \in G L(n, E):|\operatorname{det} g|_{E}=\right.$ $\left.\left|\varpi^{m}\right|_{E}\right\}$. This means that the Satake transform $\mathcal{S}(f)$ is a homogeneous Laurent polynomial of 
degree $m$. Thus $\mathcal{S}(b(f))$ is homogeneous of degree $2 m$, that is, the function $b(f)$ is supported on the set $\left\{g \in G L(n, F):|\operatorname{det} g|_{F}=\left|\varpi^{2 m}\right|_{F}\right\}$. Next the functions

$$
X \mapsto \mathbf{1}_{\left\{X:|\operatorname{det} X|_{E}=\left|\varpi^{-2 m}\right|_{E}\right\}} \int_{G L(n, E)} \Psi_{0}\left({ }^{t} \bar{g} X g\right) f(g) d g
$$

and

$$
X \mapsto \mathbf{1}_{\left\{X:|\operatorname{det} X|_{F}=\left|\varpi^{-2 m}\right|_{F}\right\}} \int_{G L(n, F)} \Phi_{0}(X g) b(f)(g) d g
$$

match because the determinant is constant on the orbits. In other words, the functions

$$
\int_{G L(n, E)} \Psi^{1}\left({ }^{t} \bar{g} X g\right) f(g) d g
$$

and

$$
\int_{G L(n, F)} \Phi^{1}(X g) b(f)(g) d g
$$

match, where we have denoted by $\Psi^{1}$ and $\Phi^{1}$ the characteristic functions of $K_{E} \cap H(n \times n$, $E / F)$ and $K_{F}$ respectively. We have established this when $f$ is supported on a set $\{g \in$ $\left.G L(n, E):|\operatorname{det} g|_{E}=\left|\varpi^{m}\right|_{E}\right\}$. By linearity, it is true for all $f$.

Equivalently, for any $f$, the functions

$$
\int_{G L(n, E)} \Psi^{1}\left({ }^{t} \bar{g} X g\right) f\left(g^{-1}\right) d g
$$

and

$$
\int_{G L(n, F)} \Phi^{1}(X g) b(f)\left(g^{-1}\right) d g
$$

match. This is the result established by Ngô in the case of positive characteristic in [15]. With minor modifications in the global theory our proof applies to that case as well.

The above result (for general Hecke functions) was first established by Ye Yangbo in the case $n=3$. His slightly different approach to the problem influenced me, as well as the notes he sent me. The reduction formulas are directly borrowed from his notes.

I am very grateful to Erez Lapid for useful discussions and a careful reading of the manuscript. His insistence that a global argument could be used to finish the proof was very encouraging. Moreover, his result in [10] is used in an essential way in the present paper. I also thank the referee for a very careful reading of the manuscript.

The material is arranged as follows. In Section 3 we explain the global consequences of our main result. This will serve as an introduction. In Section 4 we reformulate the result as a matching of linear combination of characteristic functions of lattices, namely

$$
\Psi_{\mu} \leftrightarrow \sum_{\lambda} \theta_{\mu}^{\lambda} \delta_{F}^{1 / 2}\left(\varpi^{2 \mu-\lambda}\right) \Phi_{\lambda}(X)
$$


with specific constants $\theta_{\mu}^{\lambda}$ (see below for notation). In Sections 5-11 we develop the machinery which allows us to conclude that there is a matching of this form, where the constants $\theta_{\mu}^{\lambda}$ are replaced by unknown constants $\xi_{\mu}^{\lambda}$. This amounts to saying there is a linear map $\beta: \mathcal{H}_{E} \rightarrow \mathcal{H}_{F}$ such that

$$
X \mapsto \int_{G L(n, E)} \Psi_{0}\left({ }^{t} \bar{g} X g\right) f(g) d g
$$

and

$$
X \mapsto \int_{G L(n, F)} \Phi_{0}(X g) \beta(f)(g) d g
$$

match. The assertion that $\theta_{\mu}^{\lambda}=\xi_{\mu}^{\lambda}$ is then equivalent to the equality $\beta=b$. We check that $\beta$ coincides with $b$ on the unit element and on a set of generators of the Hecke algebra in Section 12. To finish the proof we discuss a simple trace formula in Section 13, where the base change $b$ is replaced by the linear map $\beta$. We conclude in Section 14 that $\beta$ is an algebra homomorphism. Thus $\beta=b$. In Section 15 we discuss three lemmas about local distributions. In Section 16 we briefly discuss more precise global results. We also indicate how to remove the restrictions on the global quadratic extension of Section 2.

\section{General matching}

For the convenience of the reader we review the general notion of matching [6, (5) and (6)]. Thus we let $E / F$ be an arbitrary quadratic extension of non-Archimedean local fields. We let $\psi_{F}$ or simply $\psi$ be a non-trivial additive character of $F$. Then $d x$ is the self dual Haar measure on $F$ and $d z$ the Haar measure on $E$ self dual with respect to the character $\psi_{E}(z)=\psi_{F}(z+\bar{z})$. by

We let $S(F)$ be the space of invertible Hermitian matrices. The group $G(E)$ operates on $S(F)$

$$
s \mapsto{ }^{t} \bar{g} s g .
$$

For $\Psi \in C_{c}^{\infty}(S(F))$ we define as before the diagonal orbital integral

$$
\Omega[\Psi, E / F, \psi: a]
$$

The measure $d u$ on $N_{n}(E)$ is the product of the self dual Haar measures $d u_{i, j}$. More generally, we say that an element $s$ of $S(F)$ (or its orbit under $N(E)$ ) is relevant if the character

$$
u \mapsto \sum_{i} \psi\left(u_{i, i+1}+\bar{u}_{i, i+1}\right)
$$

from $N(E)$ to $F$ is trivial on the stabilizer of $s$ in $N(E)$. A system of representatives for the relevant orbits consists of the elements $w_{M} a$. Here $M$ is a standard Levi-subgroup of $G L(n)$ containing $A$, that is, $M$ is a bloc diagonal product of general linear groups. The group $A_{M} \subseteq A$ is the center of $M$, the matrix belongs to $A_{M}$ and $w_{M}$ the longest element of the Weyl group of

$4^{\mathrm{e}}$ SÉRIE - TOME $38-2005-\mathrm{N}^{\circ} 4$ 
$M$, viewed as a group of permutation matrices. In particular $w_{G}$ also noted $w_{n}$ is the matrix

$$
w_{n}:=\left(\begin{array}{cccccc}
0 & 0 & \ldots & 0 & 0 & 1 \\
0 & 0 & \ldots & 0 & 1 & 0 \\
0 & 0 & \ldots & 1 & 0 & 0 \\
\ldots & & & & & \\
0 & 1 & \ldots & 0 & 0 & 0 \\
1 & 0 & \ldots & 0 & 0 & 0
\end{array}\right)
$$

If $M$ is of type $\left(n_{1}, n_{2}, \ldots, n_{r}\right)$ with $n_{1}+n_{2}+\cdots+n_{r}=n$ then $M$ consists of diagonal matrices with block entries of size $\left(n_{1}, n_{2}, \ldots, n_{r}\right)$. The matrix $w_{M}$ is the matrix

$$
w_{M}:=\left(\begin{array}{cccccc}
w_{n_{1}} & 0 & \ldots & 0 & 0 & 0 \\
0 & w_{n_{2}} & 0 & \ldots & 0 & 0 \\
\ldots & & & & & \\
0 & 0 & \ldots & 0 & w_{n_{r-1}} & 0 \\
0 & 0 & \ldots & 0 & 0 & w_{n_{r}}
\end{array}\right)
$$

We define orbital integrals

$$
\left.\Omega\left[\Psi, \psi: w_{M} a\right]=\int \Psi{ }^{t} \bar{u} w_{M} a u\right] \theta(u \bar{u}) d u .
$$

The integral is over the quotient of $N(E)$ by the stabilizer of $w_{M} a$.

Similarly we let the group $G(F) \times G(F)$ operate on $G(F)$ by

$$
g \mapsto{ }^{t} g_{1} g g_{2} .
$$

We define the relevant elements of $G L(n, F)$ for the action of $N(F) \times N(F)$. The above matrices $w_{M} a$ form again a system of representatives for the relevant orbits. We define orbital integrals

$$
\Omega\left[\Phi, \psi: w_{M} a\right]=\int \Phi\left[{ }^{t} u_{1} w_{M} a u_{2}\right] \theta\left(u_{1} u_{2}\right) d u_{1} d u_{2} .
$$

The integral is over the quotient of $N(F)^{2}$ by the stabilizer of $w_{M} a$.

We say that $\Psi$ matches $\Phi$ and we write $\Psi \leftrightarrow \Phi$ if

$$
\Omega[\Psi, \psi: a]=\gamma(a) \Omega[\Phi, \psi: a]
$$

where $\gamma(a)$ is defined as before. Then there are transfer factors $\gamma\left(w_{M} a, \psi\right)$ such that

$$
\Omega\left[\Psi, \psi: w_{M} a\right]=\gamma\left(w_{M} a, \psi\right) \Omega\left[\Psi, \psi: w_{M} a\right] .
$$

Thus the factor $\gamma(a, \psi)=\gamma(a)$ does not depend on $\psi$. Every $\Psi$ matches a $\Phi$ and conversely.

We also need the same notions for the trivial case where $E$ is replaced by $F \oplus F$ with Galois action $\sigma\left(x_{1}, x_{2}\right)=\left(x_{2}, x_{1}\right)$. The space $S(F)$ is now the set of pairs $\left(s_{1}, s_{2}\right)$ with $s_{1}={ }^{t} s_{2}$. The action of $G L(n, E)=G L(n, F) \times G L(n, F)$ is by

$$
\left(s_{1}, s_{2}\right) \mapsto\left({ }^{t} g_{2} s_{1} g_{1},{ }^{t} g_{1} s_{2} g_{2}\right) .
$$


We say that $\Psi \in C_{c}^{\infty}(S(F))$ and $\Phi \in C_{c}^{\infty}(G(F))$ match if

$$
\int \Psi\left[{ }^{t} n_{2} a n_{1},{ }^{t} n_{1} a n_{2}\right] \theta\left(n_{1} n_{2}\right) d n_{1} d n_{2}=\int \Phi\left[{ }^{t} n_{2} a n_{1}\right] \theta\left(n_{1} n_{2}\right) d n_{1} d n_{2} .
$$

This condition is verified if

$$
\Phi(g)=\Psi\left({ }^{t} g, g\right)
$$

or

$$
\Phi(g)=\Psi\left(g,{ }^{t} g\right)
$$

The other orbital integrals are then equal.

\section{Applications}

Our main result plus the recent work of Lapid [10] on the fine spectral expansion of the relative trace formula imply the following statement. Let $E / F$ be a quadratic extension of number fields. We let $\eta_{E / F}$ or simply $\eta$ be the quadratic idele class character of $F$ attached to $E$. We fix a nontrivial character $\psi$ of $F_{\mathbb{A}} / F$. For technical reasons, we assume for now that every real place of $F$ splits in $E$. If $H$ is a unitary group in $n$-variables, and $\Pi$ an automorphic cuspidal representation of $G L\left(n, E_{\mathbb{A}}\right)$ we say that $\Pi$ is distinguished by $H$ if there is an automorphic form $\phi$ in the space of $\Pi$ such that

$$
\int_{H(F) \backslash H\left(F_{\AA}\right)} \phi(h) d h \neq 0 .
$$

We have then the following result.

THEOREM 2. - Suppose that $\Pi$ is the base change of an automorphic cuspidal representation $\pi$ of $G L\left(n, F_{\mathbb{A}}\right)$. Then there is a unitary group $H$ such that $\Pi$ is distinguished by $H$. Conversely, if an automorphic cuspidal representation $\Pi$ of $G L\left(n, E_{\mathbb{A}}\right)$ is distinguished by some unitary group then it is the base change of an automorphic cuspidal representation $\pi$ of $G L\left(n, F_{\mathbb{A}}\right)$.

We first prove the second statement. Indeed, suppose that $\Pi$ is distinguished by the stabilizer $H$ of an element $\xi \in S(F)$. If $v_{1}, v_{2}$ are places of $E$ above the same place $v$ of $F$ then the tensor product $\Pi_{v_{1}} \otimes \Pi_{v_{2}}$ admits a non-zero linear form $\mu$ invariant under the group of pairs $\left(\xi_{1}^{-1 t} h^{-1} \xi_{1}, h\right)$. Since $\left.g \mapsto \Pi_{v_{1}}{ }^{t} g^{-1}\right)$ is contragradient to $\Pi_{v_{1}}$ we conclude that $\Pi_{v_{1}}=\Pi_{v_{2}}$. On the other hand, if $v$ is a place of $F$ inert in $F, w$ the corresponding place of $E$ and $\Pi_{w}$ is unramified, then $\Pi_{w}$ is invariant under the non-trivial element of $\operatorname{Gal}\left(E_{w} / F_{v}\right)$. Thus $\Pi$ and $\Pi^{\sigma}$ have the same components at almost all places. It follows that $\Pi=\Pi^{\sigma}$. By [1] $\Pi$ is then the base change of a cuspidal automorphic representation of $G L\left(n, F_{\mathbb{A}}\right)$. (This is a standard argument first found in [5].)

We describe in more detail the (relative) trace formula which gives this result. The method of proof we follow is perhaps not the simplest. However it can be adapted to the case of the simple but delicate relative trace formula that we need to prove completely our main result.

We let $F^{+}$be the subgroup of $F_{\AA}^{\times}$whose finite components are 1 and whose infinite components are all equal to the same positive number. Thus $F^{+}$is isomorphic to $\mathbb{R}^{\times+}$. For $z \in F^{+}$we often write $z$ for $z 1_{n}$. We define $E^{+}$similarly.

$4^{\mathrm{e}}$ SÉRIE - TOME $38-2005-\mathrm{N}^{\circ} 4$ 


\subsection{The relative trace formula over $E / F$}

We denote by $S$ the algebraic variety of $n \times n$ invertible Hermitian matrices. Let $\Psi$ be a smooth function of compact support on the space $S\left(F_{\mathbb{A}}\right)$. We consider the function

$$
K_{\Psi}(g):=\sum_{\epsilon \in S(F)} \Psi\left[{ }^{t} \bar{g} \epsilon g\right]
$$

and the integral

$$
\begin{aligned}
& \int_{E^{+}} \int_{N_{n}(E) \backslash N_{n}\left(E_{\mathrm{A}}\right)} K_{\Psi}(u z) \theta(u \bar{u}) d u d z \\
& \quad=\int_{F^{+}} \int_{N_{n}(E) \backslash N_{n}\left(E_{\mathrm{A}}\right)} \sum_{\epsilon \in S(F)} \Psi\left[^{t} \bar{u} z \epsilon u\right] \theta(u \bar{u}) d z d u .
\end{aligned}
$$

Of course the Haar measures have to be normalized suitably. In this article we are only interested in qualitative results and so we do not pay much attention to the normalization of the measures. This expression can be computed in terms of orbital integrals (for the relevant orbits). Thus it is a sum

$$
\sum_{M} \sum_{\alpha \in A_{M}(F)} \int \Omega\left[\Psi, \psi: w_{M} \alpha z\right] d z
$$

The first sum is over all Levi-subgroups $M$ of $G L(n)$ containing $A$. The global orbital integral $\Omega\left[\Psi, \psi: w_{M} \alpha z\right]$ is over the quotient of $N\left(E_{\mathbb{A}}\right)$ by the stabilizer of $w_{M} \alpha$.

We consider a set of representatives $\{\xi\}$ for the orbits of the right action of $G L(n, E)$ on $S(F)$. For each $\xi$ we denote by $H^{\xi}$ its stabilizer and we choose a function $f^{\xi}$ on $G L\left(n, E_{\mathbb{A}}\right)$, smooth of compact support, such that

$$
\int_{H \xi\left(F_{\mathrm{A}}\right)} f^{\xi}(h g) d h=\Psi\left({ }^{t} \bar{g} \xi g\right) .
$$

We introduce the usual kernel function

$$
K_{f^{\xi}}(x, y):=\sum_{\gamma \in G L(n, E)} f^{\xi}\left(x^{-1} \gamma y\right) .
$$

Then

$$
\begin{aligned}
K_{\Psi}(g) & =\sum_{\xi} \sum_{\gamma \in H^{\xi}(F) \backslash G L(n, E)} \int_{H^{\xi}\left(F_{\mathrm{A}}\right)} f^{\xi}(h \gamma g) d h \\
& =\sum_{\xi} \int_{H^{\xi}(F) \backslash H^{\xi}\left(F_{\AA}\right)} K_{f^{\xi}}(h, g) d h .
\end{aligned}
$$

Thus (9) is equal to

$$
\sum_{\xi} \int_{E^{+}} \int_{H \xi(F) \backslash H^{\xi}\left(F_{\mathrm{A}}\right)} \int_{N(E) \backslash N\left(E_{\mathrm{A}}\right)} K_{f^{\xi}}(z h, u) \theta(u \bar{u}) d h d u d z .
$$


Now we make more precise the relation between the function $\Psi$ and the functions $f^{\xi}$. We assume that $\Psi$ is decomposable. Then the functions $f^{\xi}$ are decomposable.

To that end, we consider a place $v$ of $F$ inert and unramified in $E$ and the corresponding place $w$ of $E$. We consider a $\xi$ which is in $K_{w}:=G L\left(n, \mathcal{O}_{w}\right)$. Let $\Psi_{v}^{1}$ be the characteristic function of $K_{w} \cap S\left(F_{v}\right)$ and $f_{w}$ a Hecke function. Assume that $\Psi_{v}$ has the form

$$
\Psi_{v}(X)=\int_{G_{w}} \Psi_{v}^{1}\left({ }^{t} \bar{x} X x\right) f_{w}\left(x^{-1}\right) d x .
$$

Since the set $K_{w} \cap S\left(F_{v}\right)$ is a single orbit of $K_{w}$ we can write

$$
\Psi_{v}^{1}\left({ }^{t} \bar{g} \xi g\right)=\int_{H_{v}^{\xi}} f_{w}^{1}(h g) d h
$$

where $f_{w}^{1}$ is the unit element of the Hecke algebra $\mathcal{H}_{w}$. On the other hand,

$$
f_{w}(g)=\int f_{w}^{1}(x) f_{w}\left(x^{-1} g\right) d x
$$

Thus

$$
\int_{H_{v}^{\xi}} f_{w}(h g) d h=\int \Psi_{v}^{1}\left({ }^{t} \bar{x}^{t} \bar{g} \xi g x\right) f_{w}\left(x^{-1}\right) d x=\Psi_{v}\left[{ }^{t} \bar{g} \xi g\right] .
$$

In other words we may and will take $f_{w}^{\xi}=f_{w}$.

If $v$ splits into $v_{1}, v_{2}$ then the space $S\left(F_{v}\right)$ of Hermitian matrices at the place $v$ is the set of pairs

$$
\left(g_{1}, g_{2}\right), \quad g_{2}={ }^{t} g_{1}, \quad g_{i} \in G L\left(n, F_{v}\right) .
$$

In particular $\xi$ viewed as an element of $S\left(F_{v}\right)$ has the form $\xi=\left(\xi_{1}, \xi_{2}\right),{ }^{t} \xi_{2}=\xi_{1}$. The group $H_{v}^{\xi}$ is the set of pairs $\left(h_{1}, h_{2}\right)$ with $h_{1}=\xi_{1}^{-1 t} h_{2}^{-1} \xi_{1}, h_{2} \in G L\left(n, F_{v}\right)$. The relation between $\Psi_{v}$ and the function $f_{v_{1}, v_{2}}^{\xi}$ is

$$
\Psi_{v}\left[\left({ }^{t} g_{2},{ }^{t} g_{1}\right)\left(\xi_{1}, \xi_{2}\right)\left(g_{1}, g_{2}\right)\right]=\int f_{v_{1}, v_{2}}^{\xi}\left(h_{1} g_{1}, h_{2} g_{2}\right) d h
$$

or more simply

$$
\Psi_{v}\left[{ }^{t} g \xi_{1}, \xi_{2} g\right]=\int_{G L\left(n, F_{v}\right)} f_{v_{1}, v_{2}}^{\xi}\left(\xi_{1}^{-1 t} h^{-1} \xi_{1}, h g\right) d h
$$

or, after a change of variables,

$$
\Psi_{v}\left[{ }^{t} g, g\right]=\int_{G L\left(n, F_{v}\right)} f_{v_{1}, v_{2}}^{\xi}\left(\xi_{1}^{-1 t} h^{-1}, h g\right) d h .
$$

$4^{\text {e }}$ SÉRIE - TOME $38-2005-\mathrm{N}^{\circ} 4$ 
Again if the function $g \mapsto \Psi\left[{ }^{t} g, g\right]$ is a Hecke function and $\xi_{1} \in K_{v}$ we may and will take for $f_{v_{1}, v_{2}}^{\xi}$ a Hecke function $f_{v_{1}, v_{2}}$ independent of $\xi$. Then

$$
\Psi_{v}\left[{ }^{t} g, g\right]=\int f_{v_{1}, v_{2}}^{\xi}\left(h^{-1}, h g\right) d h .
$$

Now we appeal to the result of [10]. The expression (12) admits a fine spectral expansion. We only write down the discrete part of this spectral expansion. It has the form

$$
\sum_{\xi} \sum_{\Pi} \mathcal{R}_{\Pi}^{\xi}\left(f^{\xi}\right)
$$

The sum is over all $\xi$. The representations $\Pi$ which appear are, on the one hand, the cuspidal automorphic representations and, on the other hand, certain Eisenstein automorphic representations. As usual the representations $\Pi$ are normalized by the condition that their central character is trivial on $E^{+}$. If $\Pi$ is a cuspidal automorphic representation, then the relative Bessel distribution $\mathcal{R}_{\Pi}^{\xi}$ is defined by

$$
\mathcal{R}_{\Pi}^{\xi}\left(f^{\xi}\right)=\sum_{i} \int_{H^{\xi}(F) \backslash H^{\xi}\left(F_{\AA}\right)} \Pi\left(f^{\xi}\right) \phi_{i}(h) d h \overline{\int \phi_{i}(u) \bar{\theta}(u \bar{u}) d u},
$$

where the sum is over an orthonormal basis $\left\{\phi_{i}\right\}$ of $\Pi$. If $\Pi$ is an Eisenstein automorphic representation then $n$ is even and $\Pi$ of the form $\Pi_{1} \boxplus \Pi_{1}^{\sigma}$ where $\Pi_{1}$ is a cuspidal automorphic representation of $G L\left(n / 2, E_{\mathbb{A}}\right)$ with $\Pi_{1}^{\sigma}$ not equivalent to $\Pi_{1}$. The distribution $\mathcal{R}_{\Pi}^{\xi}$ is defined similarly, except that the integral over the unitary group is now a regularized integral [10].

\subsection{The relative trace formula over $F$}

Now we consider a smooth function of compact support $\Phi$ on $G L\left(n, F_{\mathbb{A}}\right)$. We associate to $\Phi$ the usual kernel

$$
K_{\Phi}(x, y):=\sum_{\gamma \in G L(n, F)} \Phi\left(x^{-1} \gamma y\right) .
$$

We consider the expression

$$
\begin{aligned}
& \int_{F^{+}} \int_{\left(N_{n}(F) \backslash N\left(F_{\AA}\right)\right)^{2}} K_{\Phi}\left({ }^{t} u_{1}^{-1}, u_{2} z\right) \theta\left(u_{1}\right) \theta\left(u_{2}\right) d u_{1} d u_{2} d z \\
& \quad=\int_{F^{+}} \int_{\left(N_{n}(F) \backslash N\left(F_{\mathrm{A}}\right)\right)^{2}} \sum_{\epsilon \in G L(n, F)} \Phi\left[{ }^{t} u_{1} z \epsilon u_{2}\right] \theta\left(u_{1} u_{2}\right) d u_{1} d u_{2} d z .
\end{aligned}
$$

As before it can be computed in terms of orbital integrals

$$
\sum_{M} \sum_{\alpha} \int \Omega\left[\Phi, \psi: w_{M} \alpha z\right] d z
$$

The expression (14) admits a fine spectral expansion. The discrete part of it is equal to

$$
\sum_{\pi} \mathcal{B}_{\pi}(\Phi)
$$


where the sum is over all irreducible cuspidal automorphic representations $\pi$. As usual the representations $\pi$ are normalized by the condition that their central character is trivial on $F^{+}$. The Bessel distribution $\mathcal{B}_{\pi}$ is defined by

$$
\mathcal{B}_{\pi}(\Phi):=\sum_{i} \int\left(\pi(\Phi) \phi_{i}\right)\left({ }^{t} u_{1}^{-1}\right) \theta\left(u_{1}\right) d u_{1} \overline{\int \phi_{i}(u) \bar{\theta}\left(u_{2}\right) d u_{2}},
$$

where the sum is over an orthonormal basis $\left\{\phi_{i}\right\}$ of $\pi$. Here again the residual discrete spectrum does not contribute because it is degenerate.

In turn, the global Bessel function is, up to a global constant, a product of local Bessel functions $\mathcal{B}_{\pi_{v}}$ (suitably normalized).

$$
\mathcal{B}_{\pi_{v}}\left(\Phi_{v}\right)=\sum_{W_{i}}\left(\pi_{v}\left(\Phi_{v}\right) W_{i}\right)\left(w_{n}\right) \bar{W}_{i}(e) .
$$

Here $W_{i}$ is an orthonormal basis of the Whittaker model of $\pi_{v}$ and $w_{n}$ is the matrix (5).

To continue we consider a cuspidal automorphic representation $\pi^{0}$ of $G L\left(n, F_{\mathbb{A}}\right)$. If $n$ is even, we assume that $\pi^{0}$ is not automorphically induced from a cuspidal automorphic representation of $G L\left(n / 2, E_{\mathbb{A}}\right)$, or equivalently, that $\pi^{0} \nsucceq \pi^{0} \otimes \eta_{E / F}$. Thus the base change $\Pi^{0}$ of $\pi^{0}$ is cuspidal. We want to show that $\Pi^{0}$ is distinguished by some unitary group. To that end, we let $S$ be a finite set of places of $F$ containing all the infinite places, all the even places, all the inert places which are ramified in $E$, all the places $v$ where the character $\psi_{v}$ has a conductor not equal to $\mathcal{O}_{v}$, and all the finite places where $\pi^{0}$ is ramified.

We will choose the functions $\Phi_{v}$ for $v$ not in $S$ to be Hecke functions. We note that if $\Phi_{v}$ is a Hecke function and $\Phi_{v}^{1}$ the characteristic function of $K_{v}:=G L\left(n, \mathcal{O}_{v}\right)$ then

$$
\Phi_{v}(X)=\int \Phi_{v}^{1}(X g) \Phi_{v}\left(g^{-1}\right) d g .
$$

By regrouping the terms for which $\pi_{\infty}$ has a given value, our sum of Bessel distributions can be written as an absolutely convergent sum

$$
\sum c\left(\pi_{\infty}\right) \mathcal{B}_{\pi_{\infty}}\left(\Phi_{\infty}\right)
$$

It will be convenient to use a principle of infinite linear independence for such sums.

LEMMA 1. - If

$$
\sum_{\pi_{\infty}} c\left(\pi_{\infty}\right) \mathcal{B}_{\pi_{\infty}}\left(\Phi_{\infty}\right)=0
$$

for all $\Phi_{\infty}$ then all the coefficients $c\left(\pi_{\infty}\right)$ are 0 .

Proof. - We defer the proof to Section 14.

Now we consider the sum of the distributions $\mathcal{B}_{\pi}(\Phi)$ for all $\pi$ such that $\pi_{\infty}=\pi_{\infty}^{0}$, namely,

$$
\sum_{\pi_{\infty}=\pi_{\infty}^{0}} \mathcal{B}_{\pi}(\Phi)
$$

We further fix $\Phi_{v}$ for $v$ finite in $S$ so as to have

$$
\mathcal{B}_{\pi_{v}^{0}}\left(\Phi_{v}\right) \neq 0 \text {. }
$$

$4^{\mathrm{e}}$ SÉRIE - TOME $38-2005-\mathrm{N}^{\circ} 4$ 
Then the above sum is finite. We label the representations which appear in the sum $\pi^{\alpha}, 0 \leqslant \alpha \leqslant r$. They are unramified at all places $v \notin S$. Next, by the strong multiplicity one, we can choose a finite set of places $T_{1}=\left\{v_{\alpha} \mid 1 \leqslant \alpha \leqslant r\right\}$ not in $S$ with the property that

$$
\forall \alpha, \quad \pi_{v_{\alpha}}^{0} \not \pi_{v_{\alpha}}^{\alpha} .
$$

For each $\alpha>0$, we choose then a Hecke function $\Phi_{v_{\alpha}}$ such that

$$
\mathcal{S}\left(\Phi_{v_{\alpha}}\right)\left(\pi_{v_{\alpha}}^{0}\right) \neq 0, \quad \mathcal{S}\left(\Phi_{v_{\alpha}}\right)\left(\pi_{v_{\alpha}}^{\alpha}\right)=0 .
$$

With this choice of functions the above sum reduces to the single term $\mathcal{B}_{\pi^{0}}(\Phi)$. We set $S_{1}=S \cup T_{1}$. Furthermore the single term is in fact a product

$$
\mathcal{B}_{\pi^{0}}(\Phi)=c \mathcal{B}_{\pi_{\infty}^{0}}\left(\Phi_{\infty}\right) \prod_{v \notin S_{1}} \mathcal{S}\left(\Phi_{v}\right)\left(\pi_{v}^{0}\right)
$$

with $c \neq 0$.

\subsection{Comparison}

If $\Psi$ and $\Phi$ satisfy a matching condition we have the equality

$$
\begin{aligned}
& \int_{F^{+} \times N(E) \backslash N\left(E_{\mathrm{A}}\right)} K_{\Psi}(z u) \theta(u \bar{u}) d z d u \\
= & \int_{F^{+} \times\left(N(F) \backslash N\left(F_{\mathrm{A}}\right)\right)^{2}} K_{\Phi}\left({ }^{t} u_{1}^{-1}, u_{2} z\right) d z \theta\left(u_{1}\right) d u_{1} \theta\left(u_{2}\right) d u_{2}
\end{aligned}
$$

that is, the expression (14) is equal to the expression (9) (geometric identity). We now describe the matching condition in detail. We assume $\Psi$ and $\Phi$ are decomposable.

Consider first a place $v$ inert in $E$ and let $w$ be the place above $v$. Then the condition of matching $\Phi_{v} \leftrightarrow \Psi_{v}$ has been recalled in Section 2. The product of the transfer factors $\gamma\left(w_{M} \alpha, \psi_{v}\right)$ over all inert places $v$ is 1 for $\alpha \in A_{M}(F)$.

At a place $v$ which splits into $v_{1}, v_{2}$ the condition of matching is

$$
\Phi_{v}(g)=\Psi_{v}\left({ }^{t} g, g\right) .
$$

Note that this definition depends on an ordering of the set $\left\{v_{1}, v_{2}\right\}$.

At every place $v \in S_{1}$ we take a function $\Psi_{v}$ which matches the function $\Phi_{v}$ that we have chosen previously. We note that for an inert place $v \in T_{1}$ the function $\Phi_{v}$ is a Hecke function but the function $\Psi_{v}$ needs not be invariant under $K_{w}$ and the functions $f_{w}^{\xi}$ need not be Hecke functions.

Recall that for $v \notin S_{1}$ we choose $\Phi_{v}$ to be a Hecke function. We make this choice and the choice of the matching function $\Psi_{v}$ more precise.

Now suppose $v$ is not in $S_{1}$ and inert. Let $w$ be the place of $E$ above $v$. We will let $f_{w}$ be a Hecke function. We take

$$
\begin{aligned}
& \Psi_{v}[X]=\int \Psi_{w}^{1}\left[{ }^{t} \bar{x} X x\right] f_{w}\left(x^{-1}\right) d x, \\
& \Phi_{v}[X]=\int \Phi_{v}^{1}[X x] b\left(f_{w}\right)\left(x^{-1}\right) d x .
\end{aligned}
$$


Indeed our main result asserts that $\Psi_{v} \leftrightarrow \Phi_{v}$. In fact we have $\Phi_{v}=b\left(f_{w}\right)$. In particular, $\Psi_{v}$ is supported on the set of elements whose determinant is a norm of the extension $E_{w} / F_{v}$.

Now let $\Xi$ be the set of $\xi$ such that $\operatorname{det}(\xi)$ is a norm at each place $v$ inert and not in $S_{1}$. The set $\Xi$ is finite and only the elements of $\Xi$ appear in our formula. We will let $T_{2}^{\prime}$ be the (finite) set of inert places $v \notin S_{1}$ for which at least one $\xi \in \Xi$ is not in $K_{w}$, where $w$ is above $v$. If $v$ is such a place and $w$ the corresponding place of $E$ we take $f_{w}$ to be the unit element of the Hecke algebra. Then $\Phi_{v}=\Phi_{v}^{1}$ and $\Psi_{v}=\Psi_{v}^{1}$. We note that the functions $f_{w}^{\xi}$ need not be Hecke functions.

If $v$ is not in $S_{1}$ and splits into $v_{1}, v_{2}$ then $\Phi_{v}$ is a Hecke function and we take

$$
\Psi_{v}\left[{ }^{t} g, g\right]=\Phi_{v}(g) .
$$

Let $T_{2}^{\prime \prime}$ be the finite set of split places $v \notin S_{1}$ such that $\xi_{1}$ is not in $K_{v_{1}}=K_{v}$ for at least one $\xi \in \Xi$. Again, the functions $f_{v_{1}, v_{2}}^{\xi}$ need not be Hecke functions. For $v \notin T_{2}^{\prime \prime}$ we take $f_{v_{1}, v_{2}}^{\xi}=f_{v_{1}, v_{2}}$ to be a Hecke function and we get

$$
\Phi_{v}(g)=\int f_{v_{1}, v_{2}}\left(h^{-1}, h g\right) d h .
$$

Thus $\Phi_{v}$ is the Hecke function base change of $f_{v_{1}, v_{2}}$.

We now set $T_{2}=T_{2}^{\prime} \cup T_{2}^{\prime \prime}$ and $S_{2}=S_{1} \cup T_{2}$. Suppose that $v$ is not in $S_{2}$ and inert. Let $w$ be the corresponding place of $E$. Then all the functions $f_{w}^{\xi}, \xi \in \Xi$, are equal to the same Hecke function $f_{w}$ and $\Phi_{v}$ is the base change of $f_{w}$. Suppose that $v$ is not in $S_{2}$ and splits into $v_{1}, v_{2}$. Then all the functions $f_{v_{1}, v_{2}}^{\xi}, \xi \in \Xi$, are equal to the same Hecke function $f_{v_{1}, v_{2}}$ and $f_{v}$ is the base change of $f_{v_{1}, v_{2}}$. The functions $f_{w}$ and $f_{v_{1}, v_{2}}$ are arbitrary, subject to the condition that they are almost all equal to the unit element. Let us call $S_{2}^{E}$ the set of places of $E$ above a place of $S_{2}$. Thus we may set

$$
f^{S_{2}^{E}}:=\bigotimes f_{w} \otimes \bigotimes f_{v_{1}, v_{2}},
$$

the tensor product being over all finite places of $E$ not in $S_{2}^{E}$. This is an element of the Hecke algebra $\mathcal{H}^{S_{2}^{E}}$, tensor product of the Hecke algebras over all places not in $S_{2}^{E}$. Then the function

$$
\Phi^{S_{2}}:=\bigotimes_{v \notin S_{2}} \Phi_{v}
$$

is in the Hecke algebra $\mathcal{H}^{S_{2}}$. It is the base change of $f^{S_{2}^{E}}$.

The functions $\Phi$ and $\Psi$ being chosen in this way, we get the equality of (15) and (10). It follows that the two spectral expansions are equal as well. We can equate the discrete parts of the spectral expansions to get the following identity:

$$
\sum_{\pi} \mathcal{B}_{\pi}(\Phi)=\sum_{\xi} \sum_{\Pi} \mathcal{R}_{\Pi}^{\xi}\left(f^{\xi}\right)
$$

Indeed, we recall the standard argument. Fix a place $v$ of $F$ split and not in $S_{2}$. Let $v_{1}, v_{2}$ be the two places above $v$. Take $f_{v_{1}, v_{2}}=f_{v} \otimes f_{0}$, where $f_{v}$ is in $\mathcal{H}_{v}$, the Hecke algebra for $G L\left(n, F_{v}\right)$, and $f_{0}$ is the unit element of $\mathcal{H}_{v}$. Thus $f_{v}$ is the image of $f_{v_{1}, v_{2}}$ under the base change map homomorphism. The Satake transform of $f_{v}$ may be viewed as a continuous function on the set $X_{v}$ of unitary, irreducible, unramified representations of $G L\left(n, F_{v}\right) \simeq G L\left(n, F_{v_{1}}\right)$. Both spectral 
expressions can then be viewed as measures on $X_{v}$. To obtain the claimed identity we equate the discrete parts of the measures. We have just obtained the identity under some restrictions on $f_{v_{1}, v_{2}}$. Since $v$ is arbitrary, we obtain the identity in general.

The representations $\Pi$ for which the distribution $\mathcal{R}_{\Pi}^{\xi}$ is non-zero are distinguished by $H^{\xi}$. As before (see the argument after Theorem 2), if $v_{1}, v_{2}$ are above the same place $v$ then $\Pi_{v_{1}}=\Pi_{v_{2}}$ (this is a standard argument).

If $\mathcal{R}_{\Pi}^{\xi} \neq 0$ then we can identify $G L\left(n, E_{\infty}\right)$ to the product $G L\left(n, F_{\infty}\right) \times G L\left(n, F_{\infty}\right)$ and write $\Pi_{\infty}$ as tensor product $\pi_{\infty} \otimes \pi_{\infty}$. In other words $\Pi_{\infty}$ is the base change of $\pi_{\infty}$. By Lemma 21, viewed as a distribution at infinity, $\mathcal{R}_{\Pi}^{\xi}\left(f^{\xi}\right)$ is a multiple of $\mathcal{B}_{\pi_{\infty}}\left(\Phi_{\infty}\right)$. Using Lemma 1 we get

$$
\sum_{\Pi_{\infty}=\pi_{\infty}^{0} \otimes \pi_{\infty}^{0}} \sum_{\xi \in X} \mathcal{R}_{\Pi}^{\xi}\left(f^{\xi}\right)=\sum_{\pi_{\infty}=\pi_{\infty}^{0}} \mathcal{B}_{\pi}(\Phi)=\mathcal{B}_{\pi^{0}}(\Phi) .
$$

Recall we have fixed the functions $\Phi_{v}$ at all finite places in $S_{2}$. Thus the functions $f_{w}^{\xi}$ for $w$ finite in $S_{2}^{E}$ are also fixed. Thus the sum on the left is a finite sum. Since $\Phi_{v}=\Phi_{v}^{1}$ for every $v \in T_{2}$ we get from (17)

$$
\mathcal{B}_{\pi^{0}}(\Phi)=c \mathcal{B}_{\pi_{\infty}^{0}}\left(\Phi_{\infty}\right) \prod_{v \notin S_{2}} \mathcal{S}\left(\Phi_{v}\right)\left(\pi_{v}^{0}\right) .
$$

On the left-hand side of (20)

$$
f^{\xi}=f_{S_{2}^{E}}^{\xi} \otimes f^{S_{2}^{E}}
$$

Thus

$$
\mathcal{R}_{\Pi}^{\xi}\left(f^{\xi}\right)=\prod_{w \notin S_{2}^{E}} \mathcal{S}\left(f_{w}\right)\left(\Pi_{w}\right) C(\Pi, \xi)
$$

where we have set

$$
C(\Pi, \xi)=\mathcal{R}_{\Pi}^{\xi}\left(f_{S_{2}^{E}}^{\xi} \otimes \mathbf{1}^{S_{2}^{E}}\right),
$$

and $\mathbf{1}^{S_{2}^{E}}$ is the unit element of $\mathcal{H}^{S_{2}^{E}}$. The only terms which appear on the left of (20) are those $\Pi$ for which

$$
\prod_{w \notin S_{2}^{E}} \mathcal{S}\left(f_{w}\right)\left(\Pi_{w}\right)=\prod_{v \notin S_{2}} \mathcal{S}\left(\Phi_{v}\right)\left(\pi_{v}^{0}\right)
$$

By the strong multiplicity result, the only $\Pi$ satisfying this condition is $\Pi^{0}$, the base change of $\pi^{0}$. Thus $\mathcal{R}_{\Pi^{0}}^{\xi}\left(f^{\xi}\right) \neq 0$ for at least one $\xi$ and we are done.

We remark that we could also use the above trace formula to prove that a distinguished representation is a base change.

\section{The set up}

We go back to the notations of Section 1 . In particular $E / F$ is an unramified quadratic extension. We denote by $\mathcal{P}^{n}$ or simply $\mathcal{P}$ the set of $n$-tuples $\lambda \in \mathbb{Z}^{n}$ such that

$$
\lambda_{1} \geqslant \lambda_{2} \geqslant \cdots \geqslant \lambda_{n}
$$


and by $\mathcal{P}_{+}$the subset of $\lambda \in \mathcal{P}$ such that $\lambda_{n} \geqslant 0$. We set

$$
|\lambda|=\sum_{1 \leqslant i \leqslant n} \lambda_{i}, \quad \tilde{\lambda}=\left(-\lambda_{n},-\lambda_{n-1}, \ldots,-\lambda_{1}\right) .
$$

We denote by $\mathcal{P}_{m}$ the subset of $\lambda \in \mathcal{P}$ such that $|\lambda|=m$. We recall the standard partial order on $\mathcal{P}$. We write

$$
\lambda \preceq \mu
$$

if

$$
\begin{aligned}
& \lambda_{1} \leqslant \mu_{1}, \quad \lambda_{1}+\lambda_{2} \leqslant \mu_{1}+\mu_{2}, \ldots, \\
& \lambda_{1}+\lambda_{2}+\cdots+\lambda_{n-1} \leqslant \mu_{1}+\mu_{2}+\cdots+\mu_{n-1}, \\
& |\lambda|=|\mu| .
\end{aligned}
$$

If $\lambda \in \mathbb{Z}^{n}$ we set

$$
\varpi^{\lambda}=\operatorname{diag}\left(\varpi^{\lambda_{1}}, \varpi^{\lambda_{2}}, \ldots, \varpi^{\lambda_{n}}\right) .
$$

If $f$ is a Hecke function, we set, for $\lambda \in \mathbb{Z}^{n}$,

$$
\begin{aligned}
& \Phi_{f}(\lambda)=\Phi_{f}\left(\varpi^{\lambda}\right):=\int_{N_{n}(F)} f\left(u \varpi^{\lambda}\right) d u, \\
& H_{f}(\lambda):=\Phi_{f}\left(\varpi^{\lambda}\right) \delta_{F}^{-1 / 2}\left(\varpi^{\lambda}\right) .
\end{aligned}
$$

Here $\delta_{F}$ is the module of the group $A(F) N(F)$. Thus if $d u$ is the Haar measure of $N_{n}(F)$ then

$$
d\left(a u a^{-1}\right)=\delta_{F}(a) d u .
$$

The function $\lambda \mapsto H_{f}(\lambda)$ is compactly supported and symmetric and arbitrary. It is determined by its values on $\mathcal{P}$.

Let $\omega_{x}^{F}$ be the zonal spherical function of parameters $x=\left(x_{1}, x_{2}, \ldots x_{n}\right)$. We recall its definition. Let $\phi_{0}$ be the function such that

$$
\begin{aligned}
& \phi_{0}(u a k)=\delta_{F}^{1 / 2}(a)\left|a_{1}\right|^{s_{1}}\left|a_{2}\right|^{s_{2}} \cdots\left|a_{n}\right|^{s_{n}}, \\
& u \in N_{n}(F), \quad a \in A_{n}(F), \quad k \in K_{F} .
\end{aligned}
$$

Here the $s_{i} \in \mathbb{C}$ are determined by the condition $|\varpi|^{s_{i}}=x_{i}$. Then

$$
\omega_{x}^{F}(g)=\int_{K_{F}} \phi_{0}(k g) d g .
$$

For any Hecke function $f$ the Satake transform is defined by

$$
\mathcal{S}(f)(x):=\int f(g) \omega_{x}^{F}(g) d g
$$

Let $\mathbb{S}_{n}$ be the subalgebra of symmetric elements in

$$
\mathbb{C}\left[X_{1}, X_{2}, \ldots, X_{n},\left(X_{1} X_{2} \cdots X_{n}\right)^{-1}\right] .
$$

$4^{\mathrm{e}}$ SÉRIE - TOME $38-2005-\mathrm{N}^{\circ} 4$ 
The maximal spectrum $\operatorname{Spec}\left[\mathbb{S}_{n}\right]$ of $\mathbb{S}_{n}$ is thus the set of the orbits of the permutation group in $\left(\mathbf{C}^{\times}\right)^{n}$. If $\pi$ is the unramified representation which admits the spherical function $\omega_{x}^{F}$ as a matrix coefficient we also write $\mathcal{S}(f)(\pi)$ for $\mathcal{S}(f)(x)$.

Recall that

$$
\begin{aligned}
\mathcal{S}(f)(x) & =\int f(g) \phi_{0}(g) d g \\
& =\sum_{\lambda \in \mathbb{Z}^{n}} \Phi_{f}\left(\varpi^{\lambda}\right) \delta_{F}^{-1 / 2}\left(\varpi^{\lambda}\right)\left|\varpi^{\lambda_{1}}\right|^{s_{1}}\left|\varpi^{\lambda_{2}}\right|^{s_{2}} \cdots\left|\varpi^{\lambda_{n}}\right|^{s_{n}} \\
& =\sum_{\lambda \in \mathbb{Z}^{n}} \Phi_{f}\left(\varpi^{\lambda}\right) \delta_{F}^{-1 / 2}\left(\varpi^{\lambda}\right) x_{1}^{\lambda_{1}} x_{2}^{\lambda_{2}} \cdots x_{n}^{\lambda_{n}} \\
& =\sum_{\lambda \in \mathbb{Z}^{n}} H_{f}(\lambda) x^{\lambda} \\
& =\sum_{\lambda \in \mathcal{P}} H_{f}(\lambda) m_{\lambda}(x)
\end{aligned}
$$

where we have set $x^{\lambda}=x_{1}^{\lambda_{1}} x_{2}^{\lambda_{2}} \cdots x_{n}^{\lambda_{n}}$ and $m_{\lambda}$ denotes the monomial symmetric function.

If $f$ is a Hecke function on $G L(n, F)$ we set

$$
W_{f}(g):=\int_{N_{n}(F)} f(u g) \theta^{-1}(u) d u .
$$

In particular, if $g=a \in A_{n}$ then this depends only on the absolute values of the entries of $a$. This allows us to write for any $\lambda \in \mathbb{Z}^{n}$,

$$
W_{f}(\lambda):=W_{f}\left(\varpi^{\lambda}\right)
$$

Of course $W_{f}(\lambda)=0$ unless $\lambda \in \mathcal{P}$. We denote by $W_{x}^{F}$ the elementary Whittaker function of parameters $x=\left(x_{1}, x_{2}, \ldots, x_{n}\right)$. Thus

$$
W_{x}^{F}(u g)=\theta(u)^{-1} W_{x}^{F}(g), \quad W_{x}^{F}(e)=1,
$$

and, for any Hecke function $f$,

$$
\int W_{x}^{F}(h g) f(g) d g=W_{x}^{F}(h) \int f(g) \omega_{x}^{F}(g) d g .
$$

In particular at $x=e$ we get

$$
\int W_{x}^{F}(g) f(g) d g=\int f(g) \omega_{x}(g) d g
$$

Let $\sigma_{\lambda}(x)$ be the Schur function attached to a $\lambda \in \mathcal{P}$. Recall the Casselman-Shalika-Shintani formula [2,17]:

$$
W_{x}^{F}\left(\omega^{\lambda}\right)=\sigma_{\lambda}(x) \delta_{F}^{1 / 2}\left(\varpi^{\lambda}\right)
$$

The left-hand side of (24) is 


$$
\begin{aligned}
\int_{A_{n}(F)} W_{x}^{F}(a) W_{f}(a) \delta_{F}^{-1}(a) d a & =\sum_{\lambda \in \mathcal{P}} W_{x}^{F}\left(\varpi^{\lambda}\right) W_{f}(\lambda) \delta_{F}^{-1}\left(\varpi^{\lambda}\right) \\
& =\sum_{\lambda \in \mathcal{P}} \sigma_{\lambda}(x) W_{f}(\lambda) \delta_{F}^{-1 / 2}\left(\varpi^{\lambda}\right)
\end{aligned}
$$

The right-hand side of (24) is $\sum_{\lambda \in \mathcal{P}} H_{f}(\lambda) m_{\lambda}(x)$. Thus for any $r$ we have

$$
\sum_{\lambda \in \mathcal{P}_{r}} \sigma_{\lambda}(x) W_{f}(\lambda) \delta_{F}^{-1 / 2}\left(\varpi^{\lambda}\right)=\sum_{\lambda \in \mathcal{P}_{r}} m_{\lambda}(x) H_{f}(\lambda) .
$$

Now

$$
\sigma_{\lambda}=\sum_{\mu \preceq \lambda} K_{\lambda}^{\mu} m_{\mu}
$$

with $K_{\lambda}^{\lambda}=1$ and

$$
m_{\lambda}=\sum_{\mu \preceq \lambda} A_{\lambda}^{\mu} \sigma_{\mu}
$$

with $A_{\lambda}^{\lambda}=1$. Because $H_{f}$ and $W_{f}$ are compactly supported, we can write

$$
\begin{aligned}
& H_{f}(\mu)=\sum_{\lambda \succeq \mu} K_{\lambda}^{\mu} W_{f}(\lambda) \delta_{F}^{-1 / 2}\left(\varpi^{\lambda}\right), \\
& W_{f}(\lambda) \delta_{F}^{-1 / 2}\left(\varpi^{\lambda}\right)=\sum_{\mu \succeq \lambda} A_{\mu}^{\lambda} H_{f}(\mu) .
\end{aligned}
$$

In particular, given $\mu \in \mathcal{P}$, we denote by $f_{\mu}^{F}$ the unique Hecke function such that

$$
W_{f_{\mu}^{F}}(\lambda)=\delta_{\lambda, \mu} .
$$

The functions $f_{\mu}^{F}, \mu \in \mathcal{P}$, form a linear basis of the space of Hecke functions.

Then

$$
\mathcal{S}(f)(x)=\int f(g) W_{x}^{F}(g) d g=\sum_{\lambda \in \mathcal{P}} W_{f}(\lambda) \delta_{F}^{-1 / 2}\left(\varpi^{\lambda}\right) \sigma_{\lambda}(x) .
$$

In particular, for the function $f_{\lambda}^{F}$, we see that

$$
\mathcal{S}\left(f_{\lambda}^{F}\right)(x)=\delta_{F}^{-1 / 2}\left(\varpi^{\lambda}\right) \sigma_{\lambda}(x) .
$$

The same applies to $E$, with $\psi_{E}(z)=\psi_{F}(z+\bar{z})$. In particular

$$
W_{f}(a)=\int_{N(E)} f(u a) \theta^{-1}(u \bar{u}) d u .
$$

Let $b$ denote the base change homomorphism $b: \mathcal{H}(G L(E)) \rightarrow \mathcal{H}(G L(F))$. By definition

$$
\mathcal{S}(b(f))(x)=\mathcal{S}(f)\left(x^{2}\right)
$$

$4^{\text {e }}$ SÉRIE - TOME $38-2005-\mathrm{N}^{\circ} 4$ 
where on the left the Satake transform is for the group $G L(n, F)$ and on the right for the group $G L(n, E)$. Equivalently,

$$
\int_{G L(n, E)} f(g) W_{x^{2}}^{E}(g) d g=\int_{G L(n, F)} b(f)(g) W_{x}^{F}(g) d g
$$

or

$$
\int_{A_{n}(E)} W_{f}(a) W_{x^{2}}^{E}(a) \delta_{E}^{-1}(a) d a=\int_{A_{n}(F)} W_{b(f)}(a) W_{x}^{F}(a) \delta_{F}^{-1}(a) d a
$$

or

$$
\sum_{\lambda} W_{f}\left(\varpi^{\lambda}\right) W_{x^{2}}^{E}\left(\varpi^{\lambda}\right) \delta_{E}\left(\varpi^{\lambda}\right)^{-1}=\sum_{\lambda} W_{b(f)}\left(\varpi^{\lambda}\right) W_{x}^{F}\left(\varpi^{\lambda}\right) \delta_{F}\left(\varpi^{\lambda}\right)^{-1}
$$

Thus

$$
\sum_{\lambda} W_{f}(\lambda) \delta_{E}\left(\varpi^{\lambda}\right)^{-1 / 2} \sigma_{\lambda}\left(x^{2}\right)=\sum_{\lambda} W_{b(f)}(\lambda) \delta_{F}\left(\varpi^{\lambda}\right)^{-1 / 2} \sigma_{\lambda}(x) .
$$

In particular, for $f=f_{\mu}^{E}$ we get,

$$
\delta_{E}^{-1 / 2}\left(\varpi^{\mu}\right) \sigma_{\mu}\left(x^{2}\right)=\sum_{\lambda} W_{b\left(f_{\mu}^{E}\right)}(\lambda) \delta_{F}^{-1 / 2}\left(\varpi^{\lambda}\right) \sigma_{\lambda}(x) .
$$

To exploit this relation, we appeal to a simple lemma.

LEMmA 2. - For every $\lambda \in \mathcal{P}$ there are unique constants $\theta_{\lambda}^{\mu}, \mu \preceq 2 \lambda$, such that

$$
\sigma_{\lambda}\left(x^{2}\right)=\sum_{\mu \preceq 2 \lambda} \theta_{\lambda}^{\mu} \sigma_{\mu}(x) .
$$

In particular $\theta_{\lambda}^{2 \lambda}=1$.

Proof. - For the sake of completeness we give a proof. Indeed

$$
\sigma_{\lambda}(x)=\sum_{\mu \preceq \lambda} K_{\lambda}^{\mu} m_{\mu}(x) .
$$

Thus

$$
\sigma_{\lambda}\left(x^{2}\right)=\sum_{\mu \preceq \lambda} K_{\lambda}^{\mu} m_{\mu}\left(x^{2}\right) .
$$

Now

$$
m_{\mu}\left(x^{2}\right)=m_{2 \mu}(x)=\sum_{\nu \preceq 2 \mu} A_{2 \mu}^{\nu} \sigma_{\nu}(x) .
$$

Our assertion follows with

$$
\theta_{\lambda}^{\nu}=\sum_{\mu, \lambda} K_{\lambda}^{\mu} A_{2 \mu}^{\nu}
$$


The relation

$$
\sigma_{\mu}\left(x_{1}^{2}, x_{2}^{2}, \ldots, x_{n}^{2}\right)=\sum_{\lambda} W_{b\left(f_{\mu}^{E}\right)}(\lambda) \delta_{F}^{-1 / 2}\left(\varpi^{\lambda-2 \mu}\right) \sigma_{\lambda}(x)
$$

is thus equivalent to

$$
W_{b\left(f_{\mu}^{E}\right)}(\lambda) \delta_{F}^{-1 / 2}\left(\varpi^{\lambda-2 \mu}\right)=\theta_{\mu}^{\lambda}
$$

Alternatively,

$$
b\left(f_{\mu}^{E}\right)=\sum_{\lambda \preceq 2 \mu} \theta_{\mu}^{\lambda} \delta_{F}^{1 / 2}\left(\varpi^{\lambda-2 \mu}\right) f_{\lambda}^{F} .
$$

Next consider the orbital integrals of

$$
\Psi(X)=\int \Psi_{0}\left({ }^{t} \bar{g} X g\right) f(g) d g
$$

where $f$ is a Hecke function. We have

$$
\begin{aligned}
\Omega(\Psi, E / F, \psi: a) & =\int \Psi\left[{ }^{t} \bar{u} a u\right] \theta(u \bar{u}) d u \\
& =\int \Psi_{0}\left[{ }^{t} \bar{g} a g\right]\left(\int f_{E}(u g) \theta^{-1}(u \bar{u}) d u\right) d g \\
& =\int \Psi_{0}\left[{ }^{t} \bar{g} a g\right] W_{f}(g) d g .
\end{aligned}
$$

Using the Iwasawa decomposition, we get

$$
=\sum_{\lambda \in \mathcal{P}} \delta_{E}\left(\varpi^{\lambda}\right)^{-1} W_{f}(\lambda) \int \Psi_{0}\left[\varpi^{\lambda t} \bar{u} a u \varpi^{\lambda}\right] \theta(u \bar{u}) d u .
$$

This is nothing but the orbital integral of the function

$$
X \mapsto \sum_{\lambda \in \mathcal{P}} \delta_{E}\left(\varpi^{\lambda}\right)^{-1} W_{f}(\lambda) \Psi_{0}\left[\varpi^{\lambda} X \varpi^{\lambda}\right] .
$$

Likewise, the orbital integral of the function $\Phi$ defined by

$$
\Phi(X)=\int \Phi_{0}(X g) b(f)(g) d g
$$

can be computed as the orbital integral of the function

$$
X \mapsto \sum_{\lambda \in \mathcal{P}} \delta_{F}\left(\varpi^{\lambda}\right)^{-1} W_{b(f)}(\lambda) \Phi_{0}\left[X \varpi^{\lambda}\right]
$$

To prove the theorem, by linearity, we may assume that $f=f_{\mu}^{E}$. Then we will have to prove that the function

$$
X \mapsto \delta_{E}^{-1}\left(\varpi^{\mu}\right) \Psi_{0}\left(\varpi^{\mu} X \varpi^{\mu}\right)
$$

$4^{\mathrm{e}}$ SÉRIE - TOME $38-2005-\mathrm{N}^{\circ} 4$ 
matches the function

$$
X \mapsto \sum_{\lambda \preceq 2 \mu} \delta_{F}^{-1}\left(\varpi^{\lambda}\right) \delta_{F}^{1 / 2}\left(\varpi^{\lambda-2 \mu}\right) \theta_{\mu}^{\lambda} \Phi_{0}\left(X \varpi^{\lambda}\right) .
$$

We will set

$$
\begin{aligned}
& \Psi_{\mu}(X):=\Psi_{0}\left(\varpi^{\mu} X \varpi^{\mu}\right), \\
& \Phi_{\lambda}(X):=\Phi_{0}\left(X \varpi^{\lambda}\right), \\
& \Phi_{\mu}^{\prime}(X):=\sum_{\lambda} \theta_{\mu}^{\lambda} \delta_{F}^{1 / 2}\left(\varpi^{2 \mu-\lambda}\right) \Phi_{\lambda}(X) .
\end{aligned}
$$

Our main result will be the consequence of the following proposition.

PROPOSITION 1. - For every $\mu$ the functions

$$
\Psi_{\mu}(X), \quad \Phi_{\mu}^{\prime}(X)
$$

match.

Before embarking on the proof we make some formal remarks. We define the Fourier transforms of Schwartz-Bruhat functions on $M(n \times n, F)$ and $M_{n}(E / F, n)$ respectively by

$$
\begin{aligned}
& \widehat{\Phi}(X)=\int \Phi(Y) \psi\left(\operatorname{Tr}\left[-w_{n} X w_{n} Y\right]\right) d Y, \\
& \widehat{\Psi}(X)=\int \Psi(Y) \psi\left(\operatorname{Tr}\left[-w_{n} X w_{n} Y\right]\right) d Y .
\end{aligned}
$$

Then the Fourier transform of $\Psi_{\mu}$ is $q^{2 n|\mu|} \Psi_{\tilde{\mu}}$. Likewise the Fourier transform of $\Phi_{\lambda}$ is $q^{n|\lambda|} \Phi_{\tilde{\lambda}}$. Also the Fourier transform of $\Phi_{\mu}^{\prime}$ is $q^{2 n|\tilde{\mu}|} \Phi_{\tilde{\mu}}^{\prime}$. For the last relation we need the fact that

$$
\theta_{\mu}^{\lambda}=\theta_{\tilde{\mu}}^{\tilde{\lambda}}
$$

Now let us make some remarks about the action of the scalar matrices $z \in F^{\times}$. We have $\gamma(a z)=\gamma(a) \gamma(z)$. Thus if $\Psi \leftrightarrow \Phi$ then the functions

$$
X \mapsto \Psi(X z) \gamma(z), \quad X \mapsto \Phi(X z)
$$

match. In particular the functions

$$
X \mapsto \Psi\left(X z^{2}\right), \quad X \mapsto \Phi\left(X z^{2}\right)
$$

match. As a consequence if we know the proposition for

$$
\mu=\left(\mu_{1}, \mu_{2}, \ldots, \mu_{n}\right)
$$

we also know the proposition for

$$
\left(\mu_{1}+m, \mu_{2}+m, \ldots, \mu_{n}+m\right)
$$

for any $m \in \mathbb{Z}$. In particular, we may assume $\mu_{n}=0$.

ANNALES SCIENTIFIQUES DE L'ÉCOLE NORMALE SUPÉRIEURE 


\section{Reduction formula}

Consider an element of $\mathcal{P}^{n}$ of the form $(\mu, \nu)$ with $\mu \in \mathcal{P}^{r}, \nu \in \mathcal{P}^{s}$ and $n=r+s$. We first prove the following reduction formula. We let $\epsilon$ be the matrix with $r$ columns and $s$ rows such that

$$
\theta\left(\begin{array}{cc}
1_{r} & X \\
0 & 1_{s}
\end{array}\right)=\psi(\operatorname{Tr}(\epsilon X))
$$

Thus

$$
\epsilon=\left(\begin{array}{cccc}
0 & \ldots & 0 & 1 \\
0 & \ldots & 0 & 0 \\
\ldots & \ldots & \ldots & \ldots \\
0 & \ldots & 0 & 0
\end{array}\right) .
$$

Likewise we let $\eta$ be the transpose of $\epsilon$. Then

$$
\theta\left[\left(\begin{array}{cc}
1_{r} & 0 \\
Y & 1_{s}
\end{array}\right)^{t}\right]=\psi(\operatorname{Tr}(Y \eta))
$$

Lemma 3. - For $A \in G L(|\mu|, F)$ and $B \in M(|\nu| \times|\nu|, F)$ set

$$
\Phi(A: B)=\int \Phi_{(\mu, \nu)}\left[\begin{array}{cc}
A & A X \\
Y A & B+Y A X
\end{array}\right] \psi(\operatorname{Tr} \epsilon X) \psi(\operatorname{Tr} Y \eta) d X d Y .
$$

If

$$
|\operatorname{det} A|=q^{|\mu|}
$$

then

$$
\Phi(A: B)=q^{-s|\mu|+r|\nu|} \Phi_{\mu}(A) \Phi_{\nu}(B) .
$$

Proof. - Explicitly

$$
\Phi(A: B)=\int \Phi_{0}\left[\begin{array}{cc}
A \varpi^{\mu} & A X \varpi^{\nu} \\
Y A \varpi^{\mu} & B \varpi^{\nu}+Y A X \varpi^{\nu}
\end{array}\right] \psi(\operatorname{Tr} \epsilon X) \psi(\operatorname{Tr} Y \eta) d X d Y .
$$

Under the assumptions of the lemma both sides of the identity to be proved are zero unless $A \varpi^{\mu} \in G L\left(r, \mathcal{O}_{F}\right)$. Assume this is the case. Then $\|Y\| \leqslant 1$. Moreover $\left\|A X \varpi^{\nu}\right\| \leqslant 1$. Hence $\left\|Y A X \varpi^{\nu}\right\| \leqslant 1$. Moreover

$$
A X \varpi^{\nu}=A \varpi^{\mu} \varpi^{-\mu} X \varpi^{\nu}
$$

Thus in fact

$$
\left\|\varpi^{-\mu} X \varpi^{\nu}\right\| \leqslant 1
$$

Writing $X=\left(x_{i, j}\right)$ we have

$$
\left|x_{i, j} \varpi^{-\mu_{i}+\nu_{j}}\right| \leqslant 1 .
$$

Since $\mu_{i} \geqslant \nu_{j}$, we have $\|X\| \leqslant 1$ which implies $\psi(\epsilon X)=1$. After integrating over $X$ and $Y$ we find

$$
q^{-s|\mu|+r|\nu|} \Phi_{0}\left[\begin{array}{cc}
A \varpi^{\mu} & 0 \\
0 & B \varpi^{\nu}
\end{array}\right]=q^{-s|\mu|+r|\nu|} \Phi_{\mu}(A) \Phi_{\nu}(B) .
$$

$4^{\mathrm{e}}$ SÉRIE - TOME $38-2005-\mathrm{N}^{\circ} 4$ 
We can compute the orbital integral of $\Phi_{(\mu, \nu)}$ as the orbital integral of $\Phi(A: B)$ on the product group $G L(r, F) \times G L(s, F)$. We arrive at the following reduction formula:

LEMMA 4. - Suppose

$$
\left|a_{1} a_{2} \cdots a_{r}\right|=\left|\operatorname{det} \varpi^{-\mu}\right|
$$

Then

$$
\begin{aligned}
& \Omega\left(\Phi_{(\mu, \nu)}, \psi: a_{1}, a_{2}, \ldots, a_{r}, a_{r+1}, a_{r+2} \ldots, a_{n}\right) \\
& \quad=q^{-s|\mu|+r|\nu|} \Omega\left(\Phi_{\mu}, \psi: a_{1}, a_{2}, \ldots, a_{r}\right) \Omega\left(\Phi_{\nu}, \psi: a_{r+1}, a_{r+2}, \ldots, a_{n}\right) .
\end{aligned}
$$

Similarly let

$$
\Psi(A: B)=\int \Psi_{(\mu, \nu)}\left[\begin{array}{cc}
t \frac{A}{X} A & B+{ }^{t} \bar{X} A X
\end{array}\right] \psi(\operatorname{Tr} \epsilon X) \psi\left(\operatorname{Tr}^{t} \bar{X} \eta\right) d X .
$$

LEMMA 5. - Suppose

$$
|\operatorname{det} A|_{F}=\left|\varpi^{-2 \mu}\right|_{F}
$$

Then

$$
\Psi(A: B)=q^{-2 s|\mu|+2 r|\nu|} \Psi_{\mu}(A) \Psi_{\nu}(B) .
$$

Proof. - Explicitly

$$
\begin{aligned}
\Psi(A: B)= & \int \Psi_{(\mu, \nu)}\left[\begin{array}{cc}
\varpi^{\mu} A \varpi^{\mu} & \varpi^{\mu} A X \varpi^{\nu} \\
\varpi^{\nu} \bar{X} A \varpi^{\mu} & \varpi^{\nu} B \varpi^{\nu}+\varpi^{\nu t} \bar{X} A X \varpi^{\nu}
\end{array}\right] \\
& \times \psi(\operatorname{Tr} \epsilon X) \psi\left(\operatorname{Tr}{ }^{t} \bar{X} \eta\right) d X .
\end{aligned}
$$

Under the assumption bot sides of the identity are 0 unless $\varpi^{\mu} A \varpi^{\mu} \in G L\left(r, \mathcal{O}_{E}\right)$. Assume this is the case. Then the matrix $\varpi^{-\mu} X \varpi^{\nu}$ is integral. It follows that the matrix

$$
\varpi^{\nu t} \bar{X} A X \varpi^{\nu}=\varpi^{\nu t} \bar{X} \varpi^{-\mu} \varpi^{\mu} A \varpi^{\mu} \varpi^{-\mu} X \varpi^{\nu}
$$

is integral. The matrix $X=\left(x_{i, j}\right)$ is itself integral with $\left|x_{i, j}\right|_{E} \leqslant\left|\varpi^{\mu_{i}-\nu_{j}}\right|_{E}$. Integrating over $X$ we get our result.

LEMMA 6. - Suppose

$$
\left|a_{1} a_{2} \cdots a_{r}\right|_{F}=\left|\operatorname{det} \varpi^{-2 \mu}\right|_{F} .
$$

Then

$$
\begin{aligned}
& \Omega\left(\Psi_{(\mu, \nu)}, E / F, \psi: a_{1}, a_{2}, \ldots, a_{r}, a_{r+1}, a_{r+2}, \ldots, a_{n}\right) \\
& \quad=q^{-2 s|\mu|+2 r|\nu|} \Omega\left(\Psi_{\mu}, E / F, \psi: a_{1}, a_{2}, \ldots, a_{r}\right) \Omega\left(\Psi_{\nu}, E / F, \psi: a_{r+1}, a_{r+2}, \ldots, a_{n}\right) .
\end{aligned}
$$

\section{The Kloosterman transform}

As in $[6,(5)]$, we introduce the normalized orbital integrals

$$
\begin{aligned}
& \widetilde{\Omega}[\Phi, \psi: a]=\left|a_{1}\right|\left|a_{1} a_{2}\right| \cdots\left|a_{1} a_{2} \cdots a_{n-1}\right| \Omega[\Phi, \psi: a], \\
& \widetilde{\Omega}[\Psi, E / F, \psi: a]= \eta\left(a_{1}\right) \eta\left(a_{1} a_{2}\right) \cdots \eta\left(a_{1} a_{2} \cdots a_{n-1}\right) \\
& \times\left|a_{1}\right|\left|a_{1} a_{2}\right| \cdots\left|a_{1} a_{2} \cdots a_{n-1}\right| \Omega[\Psi, E / F, \psi: a] .
\end{aligned}
$$

ANNALES SCIENTIFIQUES DE L'ÉCOLE NORMALE SUPÉRIEURE 
We will denote by $\mathcal{I}_{n}$ the space of functions $\omega$ on $\left(F^{\times}\right)^{n-1} \times F$ which are normalized orbital integrals, that is, of the form

$$
\omega\left(a_{1}, a_{2}, \ldots, a_{n}\right)=\widetilde{\Omega}(\Phi, \psi: a),
$$

for a suitable $\Phi \in \mathcal{S}(M(n \times n, F))$. Recall that it is also the set of normalized orbital integrals

$$
\widetilde{\Omega}[\Psi, E / F, \psi: a] .
$$

By conjugating by an appropriate diagonal matrix, we see that the space does not change if we replace $\psi$ by $\bar{\psi}$. If $\omega$ is in the space $\mathcal{I}_{n}$ its Kloosterman transform $\mathcal{K}_{n, \psi}(\omega)$ is a function in the same space. To define the Kloosterman transform, we define inductively two sequences of functions. First we set

$$
\sigma_{0}\left(a_{1}, a_{2}, \ldots, a_{n}\right):=\mu_{0}\left(a_{1}, a_{2}, \ldots, a_{n}\right):=\omega\left(a_{1}, a_{2}, \ldots, a_{n}\right) .
$$

Then we set

$$
\begin{aligned}
& \mu_{1}\left(a_{1}, a_{2}, \ldots, a_{n-1}, b_{1}\right):=\int \sigma_{0}\left(a_{1}, a_{2}, \ldots, a_{n-1}, a_{n}\right) \psi\left(-a_{n} b_{1}\right) d a_{n}, \\
& \sigma_{1}\left(a_{1}, a_{2}, \ldots, a_{n-1}, b_{1}\right)=\mu_{1}\left(a_{1}, a_{2}, \ldots, a_{n-1}, b_{1}\right) \psi\left(\frac{1}{a_{n-1} b_{1}}\right) .
\end{aligned}
$$

Inductively, if $1 \leqslant i \leqslant n-1$ and we have defined

$$
\sigma_{i}\left(a_{1}, a_{2}, \ldots, a_{n-i}, b_{i}, b_{i-1}, \ldots, b_{1}\right)
$$

then we define

$$
\begin{aligned}
& \mu_{i+1}\left(a_{1}, a_{2}, \ldots, a_{n-i-1}, b_{i+1}, b_{i}, \ldots, b_{1}\right) \\
& \quad:=\int \sigma_{i}\left(a_{1}, a_{2}, \ldots, a_{n-i}, b_{i}, b_{i-1}, \ldots, b_{1}\right) \psi\left(-a_{n-i} b_{i+1}\right) d a_{n-i}
\end{aligned}
$$

and

$$
\begin{aligned}
& \sigma_{i+1}\left(a_{1}, a_{2}, \ldots, a_{n-i-1}, b_{i+1}, b_{i}, \ldots, b_{1}\right) \\
& \quad:=\mu_{i+1}\left(a_{1}, a_{2}, \ldots, a_{n-i-1}, b_{i+1}, b_{i}, \ldots, b_{1}\right) \psi\left(\frac{1}{a_{n-i-1} b_{i+1}}\right) .
\end{aligned}
$$

In particular

$$
\sigma_{n}\left(b_{n}, b_{n-1}, \ldots, b_{1}\right):=\mu_{n}\left(b_{n}, b_{n-1}, \ldots, b_{1}\right) .
$$

Note that our definition of $\sigma_{n}$ and $\sigma_{0}$ is in accordance with the convention that an empty product has the value 1 . We emphasize that the integral defining $\mu_{i+1}$ is absolutely convergent. Moreover, for fixed $i$, the functions $\sigma_{i}$ and $\mu_{i}$ have the same support. We set then

$$
\mathcal{K}_{n, \psi}\left(b_{1}, b_{2}, \ldots, b_{n}\right)=\mu_{n}\left(b_{n}, b_{n-1}, \ldots, b_{1}\right) .
$$

For $n=1$ the Kloosterman transform is just the ordinary Fourier transform.

Just as for the ordinary Fourier transform, there is an inversion formula. More precisely, let us set

$4^{\text {e }}$ SÉRIE - TOME $38-2005-\mathrm{N}^{\circ} 4$ 


$$
\begin{aligned}
& \check{\mu}_{n-i}\left(b_{1}, b_{2}, \ldots, b_{i}, a_{n-i}, a_{n-i-1}, \ldots, a_{1}\right):=\sigma_{i}\left(a_{1}, a_{2}, \ldots, a_{n-i}, b_{i}, b_{i-1}, \ldots, b_{1}\right), \\
& \check{\sigma}_{n-i}\left(b_{1}, b_{2}, \ldots, b_{i}, a_{n-i}, a_{n-i-1}, \ldots, a_{1}\right):=\mu_{i}\left(a_{1}, a_{2}, \ldots, a_{n-i}, b_{i}, b_{i-1}, \ldots, b_{1}\right) .
\end{aligned}
$$

Then

$$
\begin{aligned}
& \check{\sigma}_{n-i}\left(b_{1}, b_{2}, \ldots, b_{i}, a_{n-i}, a_{n-i-1}, \ldots, a_{1}\right) \\
& \quad=\check{\mu}_{n-i}\left(b_{1}, b_{2}, \ldots, b_{i}, a_{n-i}, a_{n-i-1}, \ldots, a_{1}\right) \bar{\psi}\left(\frac{1}{b_{i} a_{n-i}}\right)
\end{aligned}
$$

and, from the Fourier inversion formula,

$$
\begin{aligned}
& \int \check{\sigma}_{n-i-1}\left(b_{1}, b_{2}, \ldots, b_{i}, b_{i+1}, a_{n-i-1}, \ldots a_{1}\right) \psi\left(b_{i+1} a_{n-i}\right) d b_{i+1} \\
& \quad=\check{\mu}_{n-i}\left(b_{1}, b_{2}, \ldots, b_{i}, a_{n-i}, a_{n-i-1}, \ldots, a_{1}\right) .
\end{aligned}
$$

In particular,

$$
\check{\sigma}_{n}\left(a_{n}, a_{n-1}, \ldots, a_{1}\right)=\mu_{0}\left(a_{1}, a_{2} \ldots, a_{n}\right) .
$$

In other words, the composition $\mathcal{K}_{n, \bar{\psi}} \circ \mathcal{K}_{n, \psi}$ is the identity.

There is a principle of symmetry: we can exchange the variables $\left(a_{*}\right)$ and $\left(b_{*}\right)$, the left and the right, and the character $\psi$ and the character $\bar{\psi}$.

Then the main result of $[6,(5)]$ are the following identities:

$$
\begin{aligned}
& \mathcal{K}_{n, \psi} \widetilde{\Omega}[\Phi, \psi ; \bullet]=\widetilde{\Omega}[\widehat{\Phi}, \bar{\psi}: \bullet], \\
& \mathcal{K}_{n, \psi} \widetilde{\Omega}[\Psi, E / F, \psi ; \bullet]=\widetilde{\Omega}[\widehat{\Psi}, E / F, \bar{\psi}: \bullet]
\end{aligned}
$$

\section{Definition of the diagrams}

For $n=1$ the Fourier transform has the following property. If $\phi$ is supported on the set $|a| \leqslant\left|\varpi^{r}\right|$ and its Fourier transform $\hat{\phi}$ is supported on the set $|b| \leqslant\left|\varpi^{t}\right|$ and $t+r>0$ then $\phi$ and $\hat{\phi}$ are 0 . In other words the support of $\phi$ and $\hat{\phi}$ are empty so that the above support conditions are trivially verified. Our goal in this section is to extend this result to the Kloosterman transform.

To present the proof in a convenient way, we introduce the notion of a Kloosterman diagram. Each diagram has two rows consisting of indexed boxes such as

$$
\stackrel{k}{k}, \quad k
$$

where $r$ is an integer. In the bottom row, the boxes are a shorthand notation for

$$
\left|a_{1} a_{2} \cdots a_{k}\right| \leqslant\left|\varpi^{r}\right|, \quad\left|a_{1} a_{2} \cdots a_{k}\right|=\left|\varpi^{r}\right|
$$

respectively. In the top row they are a short hand notation for

$$
\left|b_{k} b_{k-1} \cdots b_{1}\right| \leqslant\left|\varpi^{r}\right|, \quad\left|b_{k} b_{k-1} \cdots b_{1}\right|=\left|\varpi^{r}\right|
$$

respectively. In each diagram the indices are increasing in the bottom row and decreasing in the top row. Indices in boxes in the same column add up to $n+1$. For consistency we introduce dummy boxes

$$
=0
$$


Thus, in the bottom row say, a diagram

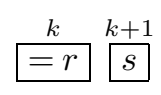

stands for

$$
\left|a_{1} a_{2} \cdots a_{k}\right|=\left|\varpi^{r}\right|, \quad\left|a_{k+1}\right| \leqslant\left|\varpi^{s-r}\right| .
$$

All our diagrams will have the following form:

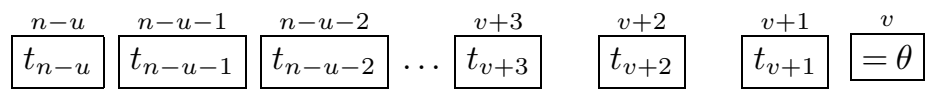

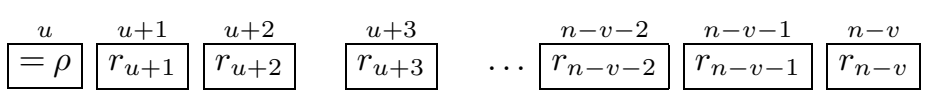

Here $0 \leqslant u \leqslant n, 0 \leqslant v \leqslant n, u+v \leqslant n$. We shall call the integer $n-u-v \geqslant 0$ the length of the diagram. We shall say that the diagram is reduced if $\rho=0, \theta=0$. The reduced diagram attached to the above diagram is the one obtained by subtracting $\theta$ from each entry in the first row and $\rho$ from each entry in the second row. The bottom row is a shorthand notation for the conditions

$$
\begin{aligned}
\left|a_{1} a_{2} \cdots a_{u}\right| & =\left|\varpi^{\rho}\right|, \\
\left|a_{1} a_{2} \cdots a_{i}\right| & \leqslant\left|\varpi^{r_{i}}\right|, \quad u+1 \leqslant i \leqslant n-v .
\end{aligned}
$$

Of course if $u=0$ then $\rho=0$, in accordance with the convention that an empty product is 1 . Likewise the top row is a shorthand notation for the conditions

$$
\begin{aligned}
& \left|b_{1} b_{2} \cdots b_{v}\right|=\left|\varpi^{\theta}\right|, \\
& \left|b_{1} b_{2} \cdots b_{i}\right| \leqslant\left|\varpi^{t_{i}}\right|, \quad v+1 \leqslant i \leqslant n-u .
\end{aligned}
$$

In what follows we let $\omega$ be a function in the space $\mathcal{I}_{n}$. We will consider such a diagram. That is, we will have chosen (sometimes only implicitly) $a_{1}, a_{2}, \ldots, a_{u}$ and $b_{1}, b_{2}, \ldots, b_{v}$ satisfying (34) and (36) respectively. We will say that the diagram holds if the conditions indicated by the bottom row hold on the support of the function

$$
\left(a_{u+1}, a_{u+2}, \ldots, a_{n-v}\right) \mapsto \mu_{v}\left(a_{1}, a_{2}, \ldots, a_{u}, a_{u+1}, a_{u+2}, \ldots, a_{n-v}, b_{v}, b_{v-1}, \ldots, b_{1}\right)
$$

while the conditions indicated by the top row hold on the support of the function

$$
\begin{aligned}
& \left(b_{n-u}, b_{n-u-1}, \ldots, b_{v+1}, b_{v}, b_{v-1}, \ldots, b_{1}\right) \\
& \quad \mapsto \mu_{n-u}\left(a_{1}, a_{2}, \ldots, a_{u}, b_{n-u}, b_{n-u-1}, \ldots, b_{v}, b_{v-1}, \ldots, b_{1}\right) .
\end{aligned}
$$

Of course we could replace $\mu_{v}$ and $\mu_{n-u}$ by $\sigma_{v}$ and $\sigma_{n-u}$ respectively. We shall say that the diagram holds trivially if the above functions are in fact identically 0 . That is, for the given $\left(a_{1}, a_{2}, \ldots, a_{u}\right)$ and $\left(b_{1}, b_{2}, \ldots, b_{v}\right)$,

$$
\mu_{v}\left(a_{1}, a_{2}, \ldots, a_{u}, a_{u+1}, a_{u+2}, \ldots, a_{n-v}, b_{v}, b_{v-1}, \ldots, b_{1}\right)=0
$$

for all $\left(a_{u+1}, a_{u+2}, \ldots, a_{n-v}\right)$ and similarly for $\mu_{n-u}$. In fact, the vanishing condition for $\mu_{v}$ implies the vanishing condition for $\mu_{n-u}$ and conversely.

A simple property of the diagrams is the following one. Suppose that the diagram (33) holds. Fix $i, v<i<n-u$. Choose $b_{i}, b_{i-1}, \ldots, b_{u+1}$ such that

$$
\left|b_{i} b_{i-1} \cdots b_{v+1}\right|=\left|\varpi^{t_{i}-\theta}\right|
$$

$4^{\mathrm{e}}$ SÉRIE - TOME $38-2005-\mathrm{N}^{\circ} 4$ 
or equivalently

$$
\left|b_{i} b_{i-1} \cdots b_{1}\right|=\left|\varpi^{t_{i}}\right|
$$

Then the following diagram holds:

$$
\begin{aligned}
& \begin{array}{c|cccccc}
n-u & n-u-1 & n-u-2 & i+2 & i+1 & i \\
\hline t_{n-u} & t_{n-u-1} & t_{n-u-2} & \ldots & t_{i+2} & t_{i+1} & =t_{i} \\
\hline
\end{array} \\
& \begin{array}{cc|ccccc}
\multicolumn{1}{c}{u} & \multicolumn{1}{c}{u+1} & u+2 & u+3 & & n-i-1 & n-i \\
\hline=\rho & r_{u+1} & r_{u+2} & r_{u+3} & \ldots & r_{n-i-1} & r_{n-i} \\
\hline
\end{array}
\end{aligned}
$$

Indeed, for the bottom row this follows from the fact that $\mu_{i}$ is obtained from $\mu_{v}$ by repeatedly multiplying by a non-zero factor and taking a Fourier transform. For the top row this is trivial. In particular, if the diagram (39) holds trivially for all choices of $\left(b_{i}, b_{i-1}, \ldots, b_{v+1}\right)$ satisfying the above condition, then the diagram (33) holds with $t_{i}$ replaced by $t_{i}+1$.

By symmetry, a similar assertion is true for a diagram of the form:

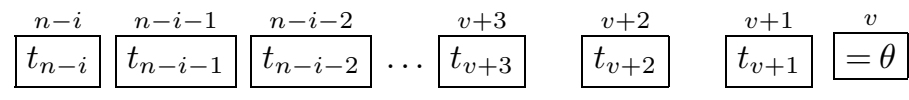

$$
\begin{aligned}
& \begin{array}{c|c|c|c|c|c|c|c|}
\multicolumn{1}{c}{i} & i+1 & u+2 & u+3 & & n-v-2 & n-v-1 & n-v \\
\hline=r_{i} & r_{i+1} & r_{u+2} & r_{u+3} & \ldots & r_{n-v-2} & r_{n-v-1} & r_{n-v} \\
\hline
\end{array}
\end{aligned}
$$

We shall say that diagrams of the form (39) or (40) are subdiagrams of the diagram (33).

\section{The weight of a diagram of length $\leqslant 2$}

We shall associate to every diagram $D$ of length an integer $w(D)$, its weight. By definition the weight of $D$ is the weight of the corresponding reduced diagram. If $D$ is reduced then $w(D)$ is the sum of the entries of $D$. If $D$ has length one, that is, has the form

$$
\stackrel{v+1}{v=\theta}
$$

$$
\stackrel{u+1}{=\rho} r
$$

with $u+v+1=n$, then

$$
w(D)=t-\theta+r-\rho .
$$

If $D$ has length 2, that is, has the form:

$$
\begin{array}{cc|c|c|} 
& v+2 & v+1 & c \\
& t_{2} & t_{1} & =\theta \\
u & & & \\
u+1 & u+2 \\
=\rho & r_{1} & r_{2} \\
\hline
\end{array}
$$

then

$$
w(D)=t_{1}-\theta+t_{2}-\theta+r_{1}-\rho+r_{2}-\rho .
$$


PROPOSITION 2. - If the weight of the diagram of length 1 or 2 is strictly positive and the diagram holds, then it holds trivially.

Before we start with the proof and the proof of a corresponding result for certain diagrams of length $n$ (Proposition 3), we remark that we are only interested in the case of a reduced diagram. We introduce the more general diagrams for the purpose of proving the propositions by induction on the length of the diagram. As a matter of fact, the assertion for a given diagram of length $n-u-v<n$ is equivalent to the similar assertion for the space $\mathcal{I}_{n-u-v}$ and the FourierKloosterman transform in $n-u-v$ variables, the integers $t_{i}$ being replaced by $t_{i}-\theta$, the integer $r_{i}$ by $r_{i}-\rho$ and the integers $\theta$ and $\rho$ by 0 .

Proof of the proposition. - We first prove the proposition for a diagram of length 1.

LEMMA 7. - Suppose that the following diagram holds:

$$
\stackrel{v+1}{v}=\theta
$$

$$
=\rho \quad u+1
$$

with $u+v+1=n$. If $r+t-\rho-\theta>0$ the diagram holds trivially.

The assumption is that the function

$$
a_{u+1} \mapsto \sigma_{v}\left(\bullet, a_{u+1}, \bullet\right)
$$

is supported on the set $\left|a_{u+1}\right| \leqslant\left|\varpi^{r-\theta}\right|$ while its Fourier transform, namely the function

$$
b_{v+1} \mapsto \mu_{v+1}\left(\bullet, b_{v+1}, \bullet\right),
$$

is supported on the set $\left|b_{v+1}\right| \leqslant\left|\varpi^{t-\theta}\right|$. The lemma follows from the uncertainty principle.

Next, we prove the proposition for a diagram of length 2 . As we explained before, this is equivalent to the proposition with $n=2$ and $\theta=\rho=0$. For clarity, we formulate this as a lemma.

LEMma 8. - Suppose $n=2$ and the following diagram holds:

$$
\begin{array}{ccc}
2 & 1 & 0 \\
t_{2} & t_{1} & =0 \\
\hline
\end{array}
$$

$$
\begin{array}{lrcr}
0 & 1 & 2 \\
=0 & r_{1} & r_{2} \\
\cline { 2 - 3 } & &
\end{array}
$$

If $r_{1}+r_{2}+t_{1}+t_{2}>0$ then the diagram holds trivially, that is, $\omega=0$.

Proof. - We recall the situation at hand. We are given

$$
\omega\left(a_{1}, a_{2}\right)=\sigma_{0}\left(a_{1}, a_{2}\right)
$$

supported on the set

$$
\left|a_{1}\right| \leqslant\left|\varpi^{r_{1}}\right|, \quad\left|a_{1} a_{2}\right| \leqslant\left|\varpi^{r_{2}}\right| .
$$

We define

$$
\mu_{1}\left(a_{1}, b_{1}\right):=\int \sigma_{0}\left(a_{1}, a_{2}\right) \psi\left(-a_{2} b_{1}\right) d a_{2}
$$

$4^{\mathrm{e}}$ SÉRIE - TOME $38-2005-\mathrm{N}^{\circ} 4$ 


$$
\begin{gathered}
\sigma_{1}\left(a_{1}, b_{1}\right)=\mu_{1}\left(a_{1}, b_{1}\right) \psi\left(\frac{1}{a_{1} b_{1}}\right), \\
\mu_{2}\left(b_{2}, b_{1}\right)=\int \sigma_{1}\left(a_{1}, b_{1}\right) \psi\left(-a_{1} b_{2}\right) d a_{1}
\end{gathered}
$$

and $\mu_{2}$ is supported on the set

$$
\left|b_{1}\right| \leqslant\left|\varpi^{t_{1}}\right|, \quad\left|b_{1} b_{2}\right| \leqslant\left|\varpi^{t_{2}}\right| .
$$

Thus, viewed as a function of $a_{1}$, the function $\sigma_{1}\left(a_{1}, b_{1}\right)$ has a module of continuity equal to $\left|b_{1} \varpi^{-t_{2}}\right|$ (i.e. is invariant under the translations belonging to $\mathcal{O}_{F} b_{1} \varpi^{-t_{2}}$ ). Similarly, viewed as a function of $b_{1}$, the function $\mu_{1}\left(a_{1}, b_{1}\right)$ has a module of continuity equal to $\left|a_{1} \varpi^{-r_{2}}\right|$.

We first observe that, by Lemma 7 , for a given $b_{1}$ with $\left|b_{1}\right|=\left|\varpi^{t_{1}+s}\right|, s \geqslant 0$, the function $\mu_{1}\left(a_{1}, b_{1}\right)$ vanishes identically unless $r_{1} \leqslant t_{1}+s-t_{2}$.

Now fix $a_{1}$ with $\left|a_{1}\right|=\left|\varpi^{r_{1}}\right|$. Let $s \geqslant 0$ be an integer such that

$$
\mu_{1}\left(a_{1}, b_{1}\right) \neq 0 \Longrightarrow\left|b_{1}\right| \leqslant\left|\varpi^{t_{1}+s}\right| .
$$

In other words, the following diagram holds:

$$
\begin{array}{lcc} 
& \multicolumn{1}{c}{1} & 0 \\
& t_{1}+s & =0 \\
1 & & \\
=r_{1} & r_{2}
\end{array}
$$

Then by Lemma $7, t_{1}+s \leqslant r_{1}-r_{2}$. From now on we restrict $b_{1}$ to the shell $\left|b_{1}\right|=\left|\varpi^{t_{1}+s}\right|$. We choose $\epsilon$ such that

$$
|\epsilon|=\left|\varpi^{t_{1}+s-t_{2}}\right|, \quad\left|a_{1}+\epsilon\right|=\left|a_{1}\right|=\left|\varpi^{r_{1}}\right|
$$

which is always possible since $t_{1}+s-t_{2} \geqslant r_{1}$ and the residual characteristic is not 2 . By the continuity of $\sigma_{1}$ we have for all $b_{1}$ in the shell

$$
\sigma_{1}\left(a_{1}+\epsilon, b_{1}\right)=\sigma_{1}\left(a_{1}, b_{1}\right),
$$

that is,

$$
\mu_{1}\left(a_{1}+\epsilon, b_{1}\right) \psi\left(\frac{1}{\left(a_{1}+\epsilon\right) b_{1}}\right)=\mu_{1}\left(a_{1}, b_{1}\right) \psi\left(\frac{1}{a_{1} b_{1}}\right) .
$$

We claim that $\mu_{1}\left(a_{1}, b_{1}\right)=0$ for all $b_{1}$ in the shell. We proceed by contradiction and fix a $b_{1}$ such that $\mu_{1}\left(a_{1}, b_{1}\right) \neq 0$. Then the above relation reads

$$
\frac{\mu_{1}\left(a_{1}+\epsilon, b_{1}\right)}{\mu_{1}\left(a_{1}, b_{1}\right)}=\psi\left(\frac{\epsilon}{\left(a_{1}+\epsilon\right) a_{1} b_{1}}\right) .
$$

Next, we let $\eta$ be such that

$$
|\eta| \leqslant\left|\varpi^{r_{1}-r_{2}}\right|, \quad\left|b_{1}+\eta\right|=\left|b_{1}\right|=\left|\varpi^{t_{1}+s}\right| .
$$


Again this is possible because $r_{1}-r_{2} \geqslant t_{1}+s$. Then

$$
\mu_{1}\left(a_{1}, b_{1}+\eta\right)=\mu_{1}\left(a_{1}, b_{1}\right), \quad \mu_{1}\left(a_{1}+\epsilon, b_{1}+\eta\right)=\mu_{1}\left(a_{1}+\epsilon, b_{1}\right)
$$

from which we get, for all such $\eta$,

$$
\psi\left(\frac{\epsilon \eta}{\left(a_{1}+\epsilon\right) a_{1}\left(b_{1}+\eta\right) b_{1}}\right)=1 .
$$

If $r_{1}-r_{2}>t_{1}+s$ then

$$
\frac{\eta}{b_{1}+\eta}
$$

is an arbitrary element of the ideal

$$
\varpi^{r_{1}-r_{2}-t_{1}-s} \mathcal{O}_{F}
$$

while

$$
\left|\frac{\epsilon}{a_{1}\left(a_{1}+\epsilon\right) b_{1}}\right|=\left|\varpi^{-2 r_{1}-t_{2}}\right| .
$$

We get then a contradiction since

$$
-2 r_{1}-t_{2}+r_{1}-r_{2}-t_{1}-s=-\left(r_{1}+r_{2}+t_{1}+t_{2}\right)-s<0 .
$$

If on the contrary $r_{1}-r_{2}=t_{1}+s$ then

$$
2 r_{1}+t_{2}=r_{1}+r_{2}+t_{1}+t_{2}+s>0
$$

and

$$
k:=\frac{\epsilon}{a_{1}\left(a_{1}+\epsilon\right) b_{1}}
$$

verifies

$$
|k|=\left|\varpi^{-t_{2}-2 r_{1}}\right|>1 .
$$

On the other hand we take $|\eta| \leqslant\left|b_{1}\right|,\left|b_{1}+\eta\right|=\left|b_{1}\right|$. Thus

$$
\frac{\eta}{b_{1}+\eta}
$$

is an integer of the form

$$
\frac{\xi}{1+\xi}, \quad|\xi| \leqslant 1, \quad|\xi+1|=1
$$

and we find that

$$
\psi\left(k \frac{\xi}{1+\xi}\right)=1
$$

for all such $\xi$. Suppose $|k| \geqslant\left|\varpi^{-2}\right|$. Any element of $\varpi \mathcal{O}_{F}$ is of the form $\xi /(1+\xi)$ with $\xi \in \varpi \mathcal{O}_{F}$. Thus we get a contradiction. If $|k|=\left|\varpi^{-1}\right|$ then in the above formula $\psi(*)$ depends 
only on $\xi$ modulo $\varpi \mathcal{O}$. Passing to the residual field $\kappa$ of $F$ we see that, for a non-trivial additive character $\psi_{0}$ of $\kappa$ we have

$$
\psi_{0}\left(\frac{\xi}{1+\xi}\right)=1
$$

for all $\xi \in \kappa, \xi \neq 0,-1$. Equivalently

$$
\psi(\theta)=1
$$

for all $\theta \neq 1$. Since

$$
\sum_{\theta \in \kappa} \psi(\theta)=0
$$

we get $\psi(1)=1-q$. This is a contradiction since $\psi(1)$ is a complex root of 1 .

We conclude that $\mu_{1}\left(a_{1}, b_{1}\right)=0$ for $\left|b_{1}\right|=\left|\varpi^{t_{1}+s}\right|$. Thus we may replace $s$ by $s+1$. Inductively, it follows that $\mu_{1}\left(a_{1}, b_{1}\right)=0$ for all $b_{1}$. Hence in fact $\mu_{1}\left(a_{1}, b_{1}\right)=0$ for all $\left|a_{1}\right|=\left|\varpi^{r_{1}}\right|$. Thus in the diagram of the lemma we may replace $r_{1}$ by $r_{1}+1$. The new diagram holds and has positive weight. Thus we may replace $r_{1}$ by an arbitrary large integer. We conclude that the support of $\omega$ is empty as claimed.

\section{Slanted diagrams}

From now on we suppress the indices in the diagrams if this does not create confusion. We shall say that a diagram is slanted with slope $m$ if it has the following form

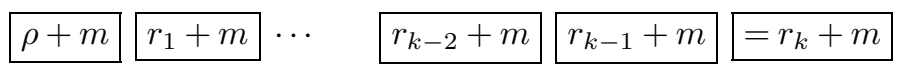

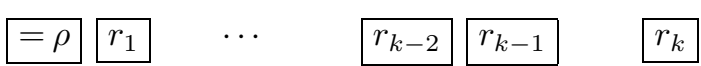

If a diagram is slanted then the corresponding reduced diagram is also slanted (with a different slope).

LEMMA 9. - Suppose that the above slanted diagram holds. Suppose further that the (nonslanted) diagram obtained by replacing the entry $r_{j}$ by $r_{j}+1$ holds. Then the slanted diagram obtained by replacing also $r_{j}+m$ by $r_{j}+m+1$ holds.

Proof. - We may assume $\rho=0$. Our assertion amounts then to saying the following. Suppose that the following diagram holds.

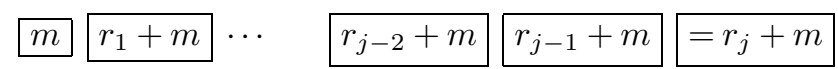

$$
\begin{aligned}
& \begin{array}{lllll}
\hline=0 & r_{1} & \cdots & r_{j-2} & r_{j-1} \\
\hline
\end{array}
\end{aligned}
$$

Then it holds trivially.

Consider first the case $j=1$. The diagram at hand has length one. and weight 1 :

$$
\begin{aligned}
& m=r_{1}+m \\
& =0 \quad r_{1}+1
\end{aligned}
$$


Thus it holds trivially. Hence we may assume $j>1$ and the result true for $j-1$. Assume that the diagram (46) holds. The right subdiagram of length 1 of diagram (46) has weight 1 thus holds trivially. Thus, in fact, the following diagram holds.

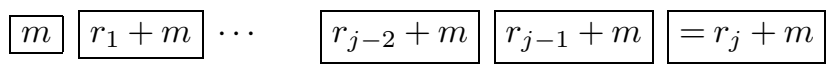

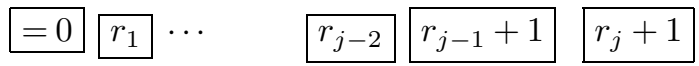

By the induction hypothesis the following diagram holds trivially.

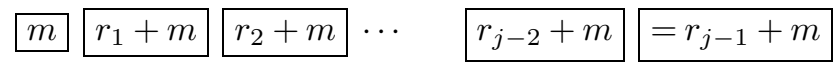

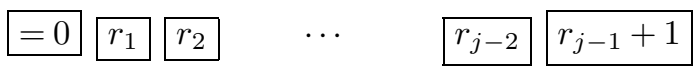

Thus in fact the following diagram holds.

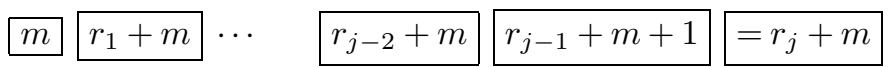

$$
\begin{aligned}
& \begin{array}{lllll}
\hline=0 & r_{1} & \cdots & r_{j-2} & r_{j-1}+1 \\
\hline
\end{array}
\end{aligned}
$$

Thus we have replaced $r_{j-1}$ in the original diagram by $r_{j-1}+1$. Inductively, we can replace $r_{j-1}$ by an arbitrarily large integer. That is, the original diagram holds trivially, as was claimed.

We will use the following elementary combinatorial lemma.

LEMmA 10. - If $D$ is slanted of length $l \geqslant 2$ then the sum of the weights of the proper subdiagrams of $D$ is equal to $\frac{l-2}{2} w(D)$.

Proof. - We may replace $D$ by the corresponding reduced diagram $D^{\prime}$. Indeed $w(D)=w\left(D^{\prime}\right)$ by definition. Moreover, if $D_{1}$ is a subdiagram of $D$ and $D_{1}^{\prime}$ the corresponding subdiagram of $D^{\prime}$ then $w\left(D_{1}\right)=w\left(D_{1}^{\prime}\right)$. Thus we may assume $D$ has the form

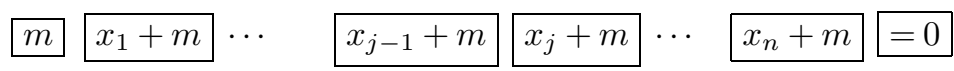

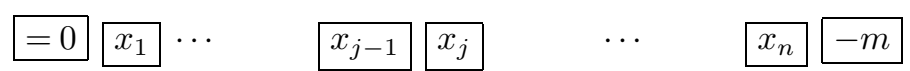

where $n=l-1$. We may regard the $x_{i}$ 's and $m$ as variables. Thus the sum of the weights of the proper subdiagrams of $D$ is a function $s\left(x_{1}, x_{2}, \ldots, x_{n} ; m\right)$. Clearly $s$ is linear:

$$
s\left(x_{1}, x_{2}, \ldots, x_{n} ; m\right)=\sum_{1 \leqslant j \leqslant n} A_{j} x_{j}+K m .
$$

To compute $A_{j}$ we remark that there are $(n-1)$ subdiagrams containing the diagonal pattern

$$
\begin{array}{ll}
\cdots & x_{j}+m \\
x_{j} & \cdots
\end{array}
$$

$4^{\mathrm{e}}$ SÉRIE - TOME $38-2005-\mathrm{N}^{\circ} 4$ 
They contribute $(n-1) 2 x_{j}$ to $s$. There is one subdiagram containing the pattern

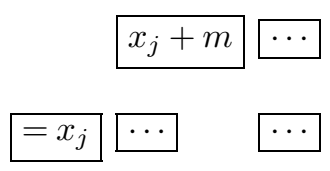

It contributes $-(n+1-j) x_{j}+x_{j}$ to $s$. There is one subdiagram with the pattern

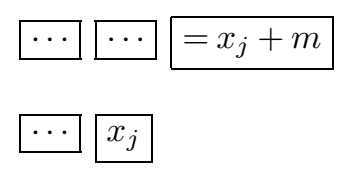

It contributes $-j x_{j}+x_{j}$ to $s$. The total contribution is $(n-1) x_{j}$. Thus $A_{j}=n-1$ for all $j$. To compute $K$ we remark that after changing $x_{j} \rightarrow x_{j}-m$ and reflecting the diagram we get

$$
s\left(x_{1}-m, x_{2}-m, \ldots, x_{n}-m ; m\right)=s\left(x_{n}, \ldots, x_{2}, x_{1} ;-m\right) .
$$

Thus

$$
K m-n(n-1) m=-K m
$$

or

$$
K=\frac{n(n-1)}{2}
$$

Thus

$$
\begin{aligned}
& s\left(x_{1}, x_{2}, \ldots, x_{n}: K\right) \\
& \quad=(n-1) \sum_{j} x_{j}+\frac{n(n-1)}{2} m=\frac{n-1}{2}\left(2 \sum x_{j}+n m\right)=\frac{l-2}{2} w(D) .
\end{aligned}
$$

Proposition 3. - Suppose that D is a slanted diagram of positive weight. If it holds, then it holds trivially.

Proof. - We already know the proposition if the length is $\leqslant 2$. Thus we may assume the length $k$ to be $\geqslant 3$ and our assertion true for any slanted diagram of shorter length. Consider a diagram $D$ of the form

$$
\begin{array}{rl|lllll} 
& \rho+m & r_{1}+m & \cdots & r_{k-2}+m & r_{k-1}+m & =r_{k}+m \\
=\rho & r_{1} & \cdots & r_{k-2} & r_{k-1} & r_{k}
\end{array}
$$

Assume that $D$ holds for a function $\omega$. Suppose that $w(D)>0$. Then, by the previous lemma, at least one of the subdiagrams of $D$ (of length $>1$ ) has positive weight, that is, holds trivially. By symmetry we may assume that it is a right subdiagram. This means that in the diagram we may increase one entry, $r_{j}$ say, by 1 and the new diagram holds. But then by Lemma 9 equation (46) we may also increase the entry $r_{j}+m$ by 1 to obtain a new slanted diagram which also holds. The weight of the new diagram has increased by 2 . Inductively we construct a sequence $D_{j}$ of slanted diagrams with increasing weight; one passes from $D_{j}$ to $D_{j+1}$ by increasing two entries 
(on the same 45 degree diagonal) by 1 . Moreover $\lim _{j \rightarrow \infty} w\left(D_{j}\right)=+\infty$. It follows that there are a subsequence $j_{s}$ of $j$ and an index $i$ independent of $s$ such that the entry in the $i$ th position in the lower line tends to infinity. This implies that $\omega=0$ and proves the proposition.

LEMMA 11. - Suppose that the following slanted diagram holds:

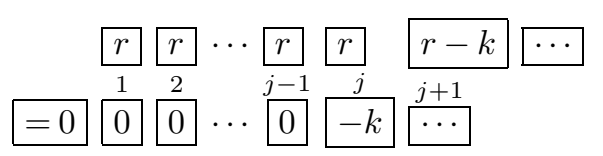

Here $j \geqslant 2, r \geqslant 0$ and $0 \leqslant k \leqslant r$. Then in fact the following diagram holds.

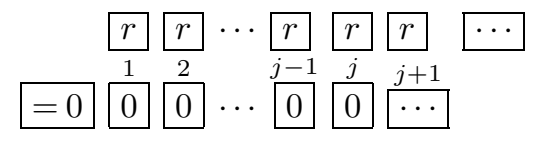

Proof. - Our assertion is vacuous if $k=0$. Thus we may assume $0<k \leqslant r$ and our assertion established for $k-1$. Consider the slanted diagram

$$
\begin{array}{rl|l|l|l|l|l|}
r & r & r & \cdots & r & r & =r-k \\
\hline=0 & 0 & 0 & \cdots & 0 & -k
\end{array}
$$

We claim it holds trivially. Indeed, the corresponding reduced diagram is

$$
\begin{array}{l|l|lll|l|l|l|l|l|l|l|l|l|}
\multicolumn{1}{l|}{} & k & k & \cdots & k & k & =0 \\
\hline=0 & 0 & 0 & \cdots & 0 & -k
\end{array}
$$

It is slanted with a positive weight so holds trivially. Thus in fact the following diagram holds:

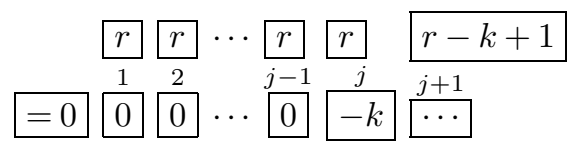

and by Lemma 9 and symmetry, the slanted diagram

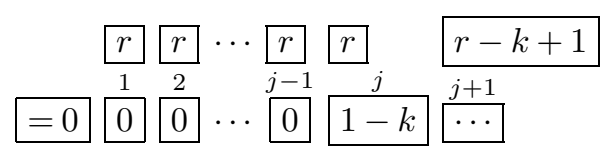

holds as well. Now we can apply the induction hypothesis to the integer $k-1$ to obtain the conclusion of the lemma.

\section{Linear independence}

In this section we prove that the orbital integrals of the functions $\Phi_{\lambda}, \lambda \in \mathcal{P}$ are linearly independent. Here and below we use the following simple but crucial fact. Let $\Delta_{r}(x)$ be the 
minor of a $n \times n$ matrix $x$ formed with the first $r$ rows and $r$ columns. Then $\Delta_{r}$ is constant on the orbits of $N(F) \times N(F)$ (or $N(E)$ ). In particular, on the support of $\Omega\left[\Phi_{\lambda}, \psi: a\right]$

$$
\left|a_{1} a_{2} \cdots a_{r}\right| \leqslant\left|\varpi^{-\lambda_{1}-\lambda_{2}-\cdots-\lambda_{r}}\right|, \quad 1 \leqslant r \leqslant n .
$$

Lemma 12. - The functions $a \mapsto \Omega\left[\Phi_{\lambda}, \psi: a\right], \lambda \in \mathcal{P}$ are linearly independent.

Proof. - Let $\mu>\lambda$ denote the total lexicographic order on $\mathcal{P}$ defined by the components. Thus

$$
\mu>\lambda
$$

means that there is an integer $s, 1 \leqslant s \leqslant n$ such that

$$
\mu_{1}=\lambda_{1}, \quad \mu_{2}=\lambda_{2}, \quad \ldots, \quad \mu_{s-1}=\lambda_{s-1}, \quad \mu_{s}>\lambda_{s} .
$$

Consider a linear relation

$$
\sum_{\lambda \in X} c_{\lambda} \Omega\left[\Phi_{\lambda}, \psi: a\right]=0
$$

where the sum is over a finite subset $X$ of $\mathcal{P}$. We have to show that all $c_{\lambda}$ are 0 . The proof is by induction on the cardinality of $X$. The statement is vacuous if $X$ is empty. Thus we may assume that $X$ is not empty and our assertion is true for a set with fewer elements. Let $\mu$ be the largest element of $X$ for the above order. Let us restrict the relation to the multishell defined by

$$
\left|a_{i}\right|=\left|\varpi^{-\mu_{i}}\right|, \quad 1 \leqslant i \leqslant n
$$

If $\lambda<\mu$ then for at least one $r \leqslant n$

$$
\lambda_{1}+\lambda_{2}+\cdots+\lambda_{r}<\mu_{1}+\mu_{2}+\cdots+\mu_{r}
$$

Since

$$
\left|a_{1} a_{2} \cdots a_{r}\right| \leqslant\left|\varpi^{-\lambda_{1}-\lambda_{2}-\cdots-\lambda_{r}}\right|
$$

on the support of $\Omega\left[\Phi_{\lambda}, \psi: a\right]$, this function vanishes on the shell. On the other hand the function $\Omega\left[\Phi_{\mu}, \psi: a\right]$ does not vanish on the shell by the reduction formula. In fact, it takes a constant nonzero value. Thus $c_{\mu}=0$. Hence we may replace the set $X$ by $X-\{\mu\}$ and apply the induction hypothesis. Our assertion follows.

LEMMA 13. - The restrictions of the functions $a \mapsto \Omega\left[\Phi_{\lambda}, \psi: a\right], \lambda \in \mathcal{P}_{m}$ to the set

$$
\left\{a:\left|a_{1} a_{2} \cdots a_{n}\right|=\left|\varpi^{-m}\right|\right\}
$$

are linearly independent.

Proof. - This follows from the proof of the previous lemma. 


\section{The main lemma}

Let $\mathcal{F}(\lambda)$ be the space of (normalized) orbital integrals $\omega$ which admit the diagram defined by $\lambda$ and $\tilde{\lambda}$, that is, the diagram

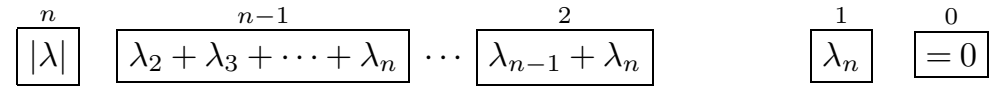

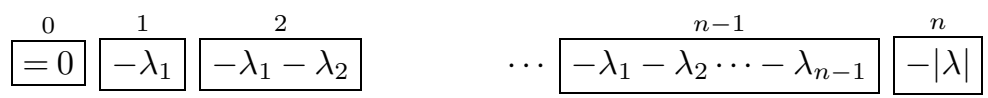

Thus the diagram is slanted with slope $|\lambda|$. Thus $\omega \in \mathcal{F}(\lambda)$ means that the function $\omega$ is supported on the set defined by

$$
\left|a_{1}\right| \leqslant\left|\varpi^{-\lambda_{1}}\right|, \quad\left|a_{1} a_{2}\right| \leqslant\left|\varpi^{-\lambda_{1}-\lambda_{2}}\right|, \quad \ldots, \quad\left|a_{1} a_{2} \cdots a_{n}\right| \leqslant\left|\varpi^{-\lambda_{1}-\lambda_{2} \cdots-\lambda_{n}}\right|,
$$

while the Kloosterman transform $\mathcal{K} \omega$ of $\omega$ is supported on the set defined by

$$
\left|b_{1}\right| \leqslant\left|\varpi^{\lambda_{n}}\right|, \quad\left|b_{1} b_{2}\right| \leqslant\left|\varpi^{\lambda_{n}+\lambda_{n-1}}\right|, \quad \ldots, \quad\left|b_{1} b_{2} \cdots b_{n}\right| \leqslant\left|\varpi^{\lambda_{n}+\lambda_{n-1}+\cdots+\lambda_{1}}\right| .
$$

If $\mu \preceq \lambda$ then $\tilde{\mu} \preceq \tilde{\lambda}$. It follows that $\mathcal{F}(\mu) \subseteq \mathcal{F}(\lambda)$.

Since the Kloosterman transform of $\widetilde{\Omega}\left[\Phi_{\mu}, \psi: a\right]$ is, up to a scalar factor, $\widetilde{\Omega}\left[\Phi_{\tilde{\mu}}, \bar{\psi}: b\right]$ we see that the function $\widetilde{\Omega}\left[\Phi_{\mu}, \psi: a\right]$ belongs to $\mathcal{F}(\mu)$ and to $\mathcal{F}(\lambda)$ for $\mu \preceq \lambda$.

In this section we prove the following key result.

PROPOSITION 4. - The functions

$$
a \mapsto \widetilde{\Omega}\left[\Phi_{\lambda^{\prime}}, \psi: a\right], \quad \lambda^{\prime} \preceq \lambda .
$$

form a linear basis of the space $\mathcal{F}(\lambda)$.

Our assertion follows from the uncertainty principle for $n=1$. Thus we may assume $n \geqslant 2$ and our assertion established for $n-1$. We note that if $\lambda=0$ then our assertion has already been established (fundamental lemma for the unit element, $[6,(8)]$ ). The space $\mathcal{F}(0)$ is then the one dimensional space spanned by $\widetilde{\Omega}\left[\Phi_{0}, \psi: a\right]$. By homogeneity, our assertion follows when $\lambda_{1}=\lambda_{n}$.

If $\lambda_{1}-\lambda_{n}=1$ then the relation $\lambda^{\prime} \preceq \lambda$ implies $\lambda^{\prime}=\lambda$. Thus the proposition asserts that $\mathcal{F}(\lambda)$ is the one dimensional space spanned by the function $a \mapsto \widetilde{\Omega}\left[\Phi_{\lambda}, \psi: a\right]$. The proof of Proposition 4 will occupy the rest of Section 11.

\subsection{The first step}

Let $\omega$ be in $\mathcal{F}(\lambda)$. Fix $a_{1}$ with $\left|a_{1}\right|=\left|\varpi^{-\lambda_{1}}\right|$ and consider the function

$$
\left(a_{2}, a_{3}, \ldots, a_{n}\right) \mapsto \omega\left(a_{1}, a_{2}, \ldots, a_{n}\right) .
$$

It is supported on the set

$$
\left|a_{2}\right| \leqslant\left|\varpi^{-\lambda_{2}}\right|, \quad\left|a_{1} a_{2}\right| \leqslant\left|\varpi^{-\lambda_{2}-\lambda_{3}}\right|, \quad \ldots, \quad\left|a_{2} a_{3} \cdots a_{n}\right| \leqslant\left|\varpi^{-\lambda_{2}-\lambda_{3}-\cdots-\lambda_{n}}\right| .
$$

$4^{\mathrm{e}}$ SÉRIE - TOME $38-2005-\mathrm{N}^{\circ} 4$ 
On the other hand, its Kloosterman transform is the function

$$
\left(b_{1}, b_{2}, \ldots, b_{n-1}\right) \mapsto \mu_{n-1}\left(a_{1}, b_{n-1}, b_{n-2}, \ldots, b_{1}\right)
$$

which is supported on the set

$$
\left|b_{1}\right| \leqslant\left|\varpi^{\lambda_{n}}\right|, \quad\left|b_{1} b_{2}\right| \leqslant\left|\varpi^{\lambda_{n}+\lambda_{n-1}}\right|, \quad \ldots, \quad\left|b_{1} b_{2} \cdots b_{n-1}\right| \leqslant\left|\varpi^{\lambda_{n}+\lambda_{n-1}+\cdots+\lambda_{2}}\right| .
$$

In other words, it belongs to the space $\mathcal{F}(\mu)$ where we have set

$$
\mu=\left(\lambda_{2}, \lambda_{3}, \ldots, \lambda_{n}\right) .
$$

By the induction hypothesis for Proposition 4, we have

$$
\omega\left(a_{1}, a_{2}, \ldots, a_{n}\right)=\sum_{\mu^{\prime} \preceq \mu} c^{\mu^{\prime}}\left(a_{1}\right) \widetilde{\Omega}\left[\Phi_{\mu^{\prime}}: a_{2}, a_{3}, \ldots, a_{n}\right],
$$

where the functions $c^{\mu^{\prime}}\left(a_{1}\right)$ are uniquely determined functions on the shell $\left|a_{1}\right|=\left|\varpi^{-\lambda_{1}}\right|$.

LEMMA 14. - With the above notation, the functions $c^{\mu^{\prime}}\left(a_{1}\right)$ are actually constant on the shell $\left|a_{1}\right|=\left|\varpi^{-\lambda_{1}}\right|$.

Proof. - To prove Lemma 14, we first prove an auxiliary lemma.

Lemma 15. - Let $\omega \in \mathcal{F}(\lambda)$. Fix $b_{n-1}, b_{n-2}, \ldots, b_{1}$ with

$$
\begin{gathered}
\left|b_{1}\right| \leqslant\left|\varpi^{\lambda_{n}}\right|, \quad\left|b_{1} b_{2}\right| \leqslant\left|\varpi^{\lambda_{n}+\lambda_{n-1}}\right|, \quad\left|b_{1} b_{2} \cdots b_{n-2}\right| \leqslant\left|\varpi^{\lambda_{n}+\lambda_{n-1}+\cdots+\lambda_{3}}\right|, \\
\left|b_{1} b_{2} \cdots b_{n-1}\right|=\left|\varpi^{|\mu|}\right| .
\end{gathered}
$$

Then the function

$$
a_{1} \mapsto \sigma_{n-1}\left(a_{1}, b_{n-1}, b_{n-2}, \ldots, b_{1}\right)
$$

is constant on the set

$$
\left|a_{1}\right| \leqslant\left|\varpi^{-\lambda_{1}}\right|
$$

and the function

$$
a_{1} \mapsto \mu_{n-1}\left(a_{1}, b_{n-1}, b_{n-2}, \ldots, b_{1}\right)
$$

is constant on the shell

$$
\left|a_{1}\right|=\left|\varpi^{-\lambda_{1}}\right|
$$

Proof. - Indeed, the function

$$
a_{1} \mapsto \sigma_{n-1}\left(a_{1}, b_{n-1}, b_{n-2}, \ldots, b_{1}\right)
$$

is supported on the set

$$
\left|a_{1}\right| \leqslant\left|\varpi^{-\lambda_{1}}\right|
$$

Under the assumption on the $b_{i}$ 's its Fourier transform, that is, the function

$$
b_{n} \mapsto \mathcal{K}(\omega)\left(b_{1}, b_{2}, \ldots, b_{n}\right)
$$

ANNALES SCIENTIFIQUES DE L'ÉCOLE NORMALE SUPÉRIEURE 
is supported on the set

$$
\left|b_{n}\right| \leqslant\left|\varpi^{\lambda_{1}}\right|
$$

The first assertion follows from the uncertainty principle. Since

$$
\sigma_{n-1}\left(a_{1}, b_{n-1}, b_{n-2}, \ldots, b_{1}\right)=\mu_{n-1}\left(a_{1}, b_{n-1}, b_{n-2}, \ldots, b_{1}\right) \psi\left(\frac{1}{a_{1} b_{n-1}}\right)
$$

for the second assertion it will suffice to prove that, on the support of the function, the factor $\psi$ is 1 for $\left|a_{1}\right|=\left|\varpi^{-\lambda_{1}}\right|$, or, what amounts to the same,

$$
\left|b_{n-1}\right| \geqslant\left|\varpi^{\lambda_{1}}\right| .
$$

Indeed

$$
\left|b_{n-1}\right|=\left|\varpi^{\lambda_{n}+\lambda_{n-1}+\cdots+\lambda_{3}+\lambda_{2}}\right|\left|b_{n-2} b_{n-3} \cdots b_{1}\right|^{-1} .
$$

Now

$$
\left|b_{n-2} b_{n-3} \cdots b_{1}\right| \leqslant\left|\varpi^{\lambda_{n}+\lambda_{n-1}+\cdots+\lambda_{3}}\right| .
$$

Hence

$$
\left|b_{n-1}\right| \geqslant\left|\varpi^{\lambda_{2}}\right| \geqslant\left|\varpi^{\lambda_{1}}\right|
$$

the last inequality because $\lambda_{1} \geqslant \lambda_{2}$. This concludes the proof of Lemma 15 .

We now go back to the problem of proving that the functions $c^{\mu^{\prime}}$ we introduced in (50) are constant. To that end, we take the Kloosterman transform of the identity (50) with respect to the variables $\left(a_{2}, a_{3}, \ldots, a_{n}\right)$. We get

$$
\mu_{n-1}\left(a_{1}, b_{n-1}, b_{n-2}, \ldots, b_{1}\right)=\sum_{\mu^{\prime} \preceq \mu} c^{\mu^{\prime}}\left(a_{1}\right) q^{n|\mu|} \widetilde{\Omega}\left[\Phi_{\tilde{\mu}^{\prime}}, \bar{\psi}: b_{1}, b_{2}, \ldots, b_{n-1}\right] .
$$

Now let us restrict this relation to the set of $b_{i}$ 's in Lemma 15. The left-hand side is independent of $a_{1}$ in the shell. On the other hand, the restriction of the functions $\widetilde{\Omega}\left[\Phi_{\tilde{\mu}^{\prime}}, \bar{\psi}: b_{1}, b_{2}, \ldots, b_{n-1}\right]$ to the set of the $b_{i}$ 's in Lemma 15 are linearly independent by Lemma 13. It follows that the functions $c^{\mu^{\prime}}\left(a_{1}\right)$ are indeed constant on the shell. This establishes Lemma 14.

To continue, we denote by $c^{\mu^{\prime}}$ their constant values of the functions $c^{\mu^{\prime}}$. Thus the relation

$$
\omega\left(a_{1}, a_{2}, \ldots, a_{n}\right)=\sum_{\mu^{\prime} \preceq \mu} c^{\mu^{\prime}} \widetilde{\Omega}\left[\Phi_{\mu^{\prime}}: a_{2}, a_{3}, \ldots, a_{n}\right]
$$

holds for $a_{1}$ in the shell $\left|a_{1}\right|=\left|\varpi^{-\lambda_{1}}\right|$. If $\mu^{\prime} \preceq \mu$ then $\lambda^{\prime}:=\left(\lambda_{1}, \mu^{\prime}\right)$ satisfies $\lambda^{\prime} \preceq \lambda$. In addition, by the reduction formula, on the shell,

$$
\widetilde{\Omega}\left[\Phi_{\lambda^{\prime}}: a_{1}, a_{2}, \ldots, a_{n}\right]=q^{|\mu|} \Omega\left[\Phi_{\mu^{\prime}}: a_{2}, a_{3}, \ldots, a_{n}\right] .
$$

After a change of notation we see that we can write, for $a_{1}$ in the shell $\left|a_{1}\right|=\left|\varpi^{-\lambda_{1}}\right|$,

$$
\omega\left(a_{1}, a_{2}, \ldots, a_{n}\right)=\sum_{\lambda^{\prime} \preceq \lambda, \lambda_{1}^{\prime}=\lambda_{1}} c^{\lambda^{\prime}} \widetilde{\Omega}\left[\Phi_{\lambda^{\prime}}: a_{1}, a_{2}, \ldots, a_{n}\right] .
$$

$4^{\mathrm{e}}$ SÉRIE - TOME $38-2005-\mathrm{N}^{\circ} 4$ 
Let us set

$$
\omega_{1}(a):=\omega(a)-\sum_{\lambda^{\prime} \preceq \lambda \lambda_{1}^{\prime}=\lambda_{1}} c^{\lambda^{\prime}} \widetilde{\Omega}\left[\Phi_{\lambda^{\prime}}: a_{1}, a_{2}, \ldots, a_{n}\right] .
$$

This function is still in $\mathcal{F}(\lambda)$. However its support is contained in the set

$$
\left|a_{1}\right| \leqslant\left|\varpi^{1-\lambda_{1}}\right|
$$

That is, the function $\omega_{1}$ admits the diagram

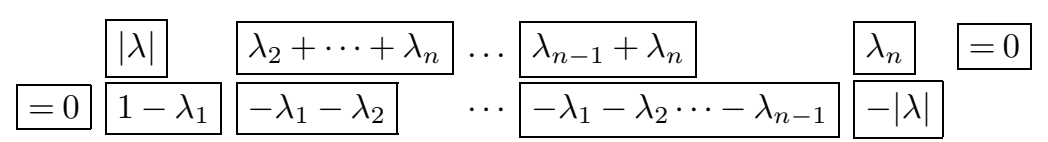

By Lemma 9 it follows that it admits the diagram

$$
\begin{array}{l|lllll|}
\multicolumn{1}{l|}{} & \multicolumn{1}{|l|}{1+\lambda_{2}+\cdots+\lambda_{n}} & \cdots & \lambda_{n-1}+\lambda_{n} & \lambda_{n} & =0 \\
\hline=0 & 1-\lambda_{1} & -\lambda_{1}-\lambda_{2} & \cdots & -\lambda_{1}-\lambda_{2} \cdots-\lambda_{n-1} & -|\lambda|
\end{array}
$$

Proposition 4 will now follow from the following lemma.

LEMMA 16. - The function $\omega_{1}$ belongs to some space $\mathcal{F}\left(\lambda^{\prime}\right)$ with $\lambda^{\prime} \prec \lambda$.

If $\lambda_{1}-\lambda_{2} \geqslant 2$ we can set

$$
\lambda^{\prime}=\left(\lambda_{1}-1, \lambda_{2}+1, \lambda_{3}, \ldots, \lambda_{n}\right) .
$$

Clearly $\lambda^{\prime}$ is still in $\mathcal{P}$. We have $\lambda^{\prime} \prec \lambda$ and the function $\omega_{1}$ is in $\mathcal{F}\left(\lambda^{\prime}\right)$. The lemma follows in this case. From now on we assume that $\lambda_{1}-\lambda_{2} \leqslant 1$.

\subsection{The case of a fundamental weight}

Suppose that

$\lambda_{1}-\lambda_{n} \leqslant 1$. Then

$$
\omega_{1}(a)=\omega(a)-c \widetilde{\Omega}\left[\Phi_{\lambda}: a\right]
$$

with $c \neq 0$. Lemma 16 amounts to saying that $\omega_{1}=0$ or, equivalently, Proposition 4 , amounts to saying that $\mathcal{F}(\lambda)$ is the one dimensional space spanned by $\widetilde{\Omega}\left[\Phi_{\lambda}: a\right]$.

Assume first $\lambda_{1}-\lambda_{n}=1$. Note that we may replace $\lambda$ by $\tilde{\lambda}$ and use homogeneity. In particular, we may assume

$$
\lambda=(\underbrace{1,1, \ldots, 1}_{r}, \underbrace{0,0, \ldots, 0}_{s}), \quad r \geqslant s .
$$

We have to show that the function $\omega_{1}$ of (51) is 0 .

For instance if

$$
\lambda=(1,1,1,1,0,0)
$$

then the diagram associated with $\lambda$ is

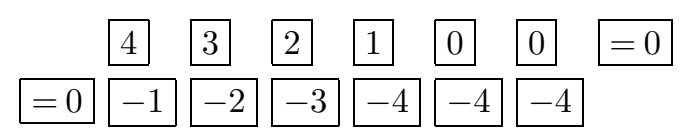


then the diagram for the function $\omega_{1}$ is

$$
\begin{array}{l|l|l|l|l|l|l|l|}
\multicolumn{1}{l|}{} & 4 & 4 & 2 & 1 & 0 & 0 & =0 \\
\hline=0 & 0 & -2 & -3 & -4 & -4 & -4 \\
\hline
\end{array}
$$

Applying repeatedly Lemma 11 we see that the following diagram holds.

$$
\begin{array}{l|l|l|l|l|l|l|l|l|l|l|} 
& 4 & 4 & 4 & 4 & 4 & 4 & =0 \\
=0 & 0 & 0 & 0 & 0 & 0 & -4
\end{array}
$$

Since this slanted diagram has positive weight it holds trivially, that is, $\omega_{1}=0$. In general, applying repeatedly Lemma 11 we see that $\omega_{1}$ admits a slanted diagram whose entries in the first row are all equal to $r$ and all entries in the last row are 0 except one which is $-r$. This diagram has positive weight and the conclusion follows again.

If $\lambda_{1}-\lambda_{n}=0$, as noted earlier, we have proved our assertion previously in [8, (8)]. For the sake of completeness we reproduce the proof. We may as well assume $\lambda=(0,0, \ldots, 0)$. If

$$
\lambda=(0,0,0,0,0,0)
$$

for instance, then the diagram associated to $\lambda$ is

$$
\begin{array}{l|l|l|l|l|l|l|l|}
\hline & 0 & 0 & 0 & 0 & 0 & 0 & =0 \\
\hline=0 & 0 & 0 & 0 & 0 & 0 & 0 \\
\hline
\end{array}
$$

and $\omega_{1}$ admits the diagram

$$
\begin{array}{l|l|l|l|l|l|l|l|l|l|l|l|l|l|l|l|l} 
& 0 & 1 & 0 & 0 & 0 & 0 & =0 \\
\hline=0 & 1 & 0 & 0 & 0 & 0 & 0
\end{array}
$$

This is a slanted diagram of slope 0 and weight 2 . Thus $\omega_{1}=0$. Thus we have proved Lemma 16 or Proposition 4 when $\lambda_{1}-\lambda_{n} \leqslant 1$.

\subsection{The second step}

Lemma 17. - Suppose that $\lambda \in \mathcal{P}^{n}$ verifies

$$
\lambda_{1}-\lambda_{r} \leqslant 1
$$

for some $r$ with $1<r \leqslant n-1$. Suppose that $\omega$ is an element of $\mathcal{F}(\lambda)$ such that $\omega=0$ for $\left|a_{1}\right|=\left|\varpi^{-\lambda_{1}}\right|$. Then $\mathcal{K} \omega=0$ for

$$
\left|b_{1} b_{2} \cdots b_{n-r}\right|=\left|\varpi^{\lambda_{n}+\lambda_{n-1}+\cdots+\lambda_{r+1}}\right|
$$

and $\omega=0$ for

$$
\left|a_{1} a_{2} \cdots a_{r}\right|=\left|\varpi^{-\lambda_{1}-\lambda_{2}-\cdots-\lambda_{r}}\right|
$$

$4^{\mathrm{e}}$ SÉRIE - TOME $38-2005-\mathrm{N}^{\circ} 4$ 
Proof. - We set

$$
\mu=\left(\lambda_{1}, \lambda_{2}, \ldots, \lambda_{r}\right), \quad \nu=\left(\lambda_{r+1}, \lambda_{r+2}, \ldots, \lambda_{n}\right) .
$$

Thus $\lambda=(\mu, \nu)$. Fix $b_{1}, b_{2}, \ldots, b_{n-r}$ with

$$
\left|b_{1}, b_{2} \cdots b_{n-r}\right|=\left|\varpi^{\lambda_{n}+\lambda_{n-1}+\cdots+\lambda_{r+1}}\right| .
$$

The function

$$
\left(a_{1}, a_{2}, \ldots, a_{r}\right) \mapsto \sigma_{n-r}\left(a_{1}, a_{2}, \ldots, a_{r}, b_{n-r}, b_{n-r-1}, \ldots, b_{1}\right)
$$

belongs to $\mathcal{F}(\mu)$. By the previous section

$$
\sigma_{n-r}\left(a_{1}, a_{2}, \ldots, a_{r}, b_{n-r}, b_{n-r-1}, \ldots, b_{1}\right)=M\left(b_{1}, b_{2}, \ldots, b_{n-r}\right) \widetilde{\Omega}\left[\Phi_{\mu}, \psi: a_{1}, a_{2}, \ldots, a_{r}\right] .
$$

By the assumption, the left-hand side vanishes if $\left|a_{1}\right|=\left|\varpi^{-\lambda_{1}}\right|$. On the other hand $\widetilde{\Omega}\left[\Phi_{\mu}, \psi\right.$ : $\left.a_{1}, a_{2}, \ldots, a_{r}\right]$ takes a non-zero constant value on the set

$$
\left|a_{1}\right|=\left|\varpi^{-\lambda_{1}}\right|, \quad\left|a_{1} a_{2}\right|=\left|\varpi^{-\lambda_{1}-\lambda_{2}}\right|, \quad\left|a_{1} a_{2} \cdots a_{r}\right|=\left|\varpi^{-\lambda_{1}-\lambda_{2}-\cdots-\lambda_{r}}\right| .
$$

Thus in fact $M$ is identically 0 and we conclude that

$$
\sigma_{n-r}\left(a_{1}, a_{2}, \ldots, a_{r}, b_{n-r}, b_{n-r-1}, \ldots, b_{1}\right)=0
$$

for $\left|b_{1}, b_{2} \cdots b_{n-r}\right|=\left|\varpi^{\lambda_{n}+\lambda_{n-1}+\cdots+\lambda_{r+1}}\right|$ which is equivalent to the first assertion. Moreover, the function $\mu_{n-r}$ has the same property.

Now fix $a_{1}, a_{2}, \ldots, a_{r}$ with

$$
\left|a_{1} a_{2} \cdots a_{r}\right|=\left|\varpi^{-\lambda_{1}-\lambda_{2}-\cdots-\lambda_{r}}\right|
$$

Then the function

$$
\left(a_{r+1}, a_{r+2} \ldots, a_{n}\right) \mapsto \omega\left(a_{1}, a_{2}, \ldots, a_{r}, a_{r+1}, \ldots, a_{n}\right)
$$

belongs to $\mathcal{F}(\nu)$. By the induction hypothesis for Proposition 4, it can be written as

$$
\sum_{\nu^{\prime} \preceq \nu} C^{\nu^{\prime}}\left(a_{1}, a_{2}, \ldots, a_{r}\right) \widetilde{\Omega}\left[\Phi_{\nu^{\prime}}, \psi ; a_{r+1}, a_{r+2}, \ldots, a_{n}\right] .
$$

Taking the Kloosterman transform of this identity we get

$$
\begin{aligned}
& \mu_{n-r}\left(a_{1}, a_{2}, \ldots, a_{r}, b_{n-r}, b_{n-r-1}, \ldots, b_{1}\right) \\
& \left.\quad=\sum_{\nu^{\prime} \preceq \nu} C^{\nu^{\prime}}\left(a_{1}, a_{2}, \ldots, a_{r}\right) q^{2(n-r)|\nu|} \widetilde{\Omega}_{\widetilde{\nu^{\prime}}}, \bar{\psi}: b_{1}, b_{2}, \ldots b_{n-r}\right] .
\end{aligned}
$$

Since this vanishes on the set

$$
\left|b_{1}, b_{2} \cdots b_{n-r}\right|=\left|\varpi^{\lambda_{n}+\lambda_{n-1}+\cdots+\lambda_{n-r+1}}\right|
$$

ANNALES SCIENTIFIQUES DE L'ÉCOLE NORMALE SUPÉRIEURE 
by the first part of the lemma and the functions

$$
\widetilde{\Omega}\left[\Phi_{\widetilde{\nu^{\prime}}}, \bar{\psi}: b_{1}, b_{2}, \ldots b_{n-r}\right]
$$

are linearly independent on that set, we conclude that the functions

$$
C^{\nu^{\prime}}\left(a_{1}, a_{2}, \ldots, a_{r}\right)
$$

all vanish. The lemma follows.

\subsection{Reduction to the case of a fundamental weight}

Now we prove Lemma 16 for a $\lambda$ such that $\lambda_{1}-\lambda_{2} \leqslant 1$ and $\lambda_{1}-\lambda_{n}>1$. Next, consider the largest integer $1<s<n$ such that $\lambda_{1}-\lambda_{s} \leqslant 1$. Thus we can write

$$
\lambda=(\mu, \nu)
$$

with

$$
\mu=\left(\lambda_{1}, \lambda_{2}, \ldots, \lambda_{s}\right), \quad \lambda_{1}-\lambda_{s} \leqslant 1, \quad \lambda_{1}-\lambda_{s+1} \geqslant 2 .
$$

By homogeneity we may assume

$$
\mu=(\overbrace{s}^{\overbrace{1,1,1, \ldots, 1}^{s_{1}}, \overbrace{0,0 \ldots, 0}^{s_{2}}})
$$

with $s_{1}>0, s_{2} \geqslant 0$ and

$$
\lambda_{s+1} \leqslant-1 .
$$

We consider an $\omega$ in $\mathcal{F}(\lambda)$. As before, we define $\omega_{1}$ by (51). Thus $\omega_{1}$ is in $\mathcal{F}(\lambda)$ and $\omega_{1}=0$ for $\left|a_{1}\right|=\left|\varpi^{-\lambda_{1}}\right|$. Then we claim that $\omega_{1}$ is in $\mathcal{F}\left(\lambda^{\prime}\right)$ where $\lambda^{\prime}$ is obtained by replacing $\lambda_{s_{1}}=1$ by $\lambda_{s_{1}}=0$ and $\lambda_{s+1}$ by $\lambda_{s+1}+1$. Of course $\lambda^{\prime} \prec \lambda$. This will prove Lemma 16 in this case. We illustrate the case $s_{1}=2, s_{2}=2, n=6$. Thus

$$
\lambda=\left(1,1,0,0, \lambda_{5}, \lambda_{6}\right)
$$

with $\lambda_{5}<0$. We set $a:=\lambda_{5}+\lambda_{6}$. The diagram associated to $\lambda$ is

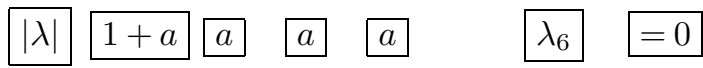

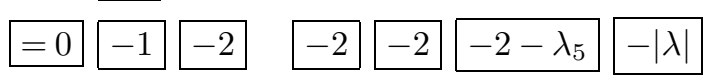

Both $\omega$ and $\omega_{1}$ admit this diagram. But by the previous lemma for $r=2,3,4$ the function $\omega_{1}$ admits in fact the diagram

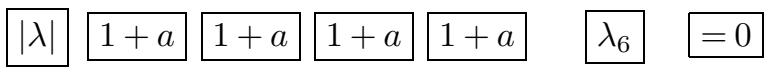

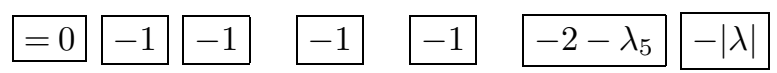

$4^{\mathrm{e}}$ SÉRIE - TOME $38-2005-\mathrm{N}^{\circ} 4$ 
This is the diagram attached to $\lambda^{\prime}$ where

$$
\lambda^{\prime}=\left(1,0,0,0, \lambda_{5}+1, \lambda_{6}\right)
$$

Hence $\omega_{1} \in \mathcal{F}\left(\lambda^{\prime}\right)$. In general we apply the previous lemma with $s_{1} \leqslant r \leqslant s$.

This concludes the proof of Lemma 16 and hence the proof of Proposition 4.

\section{The map $\beta$}

Recall that the Kloosterman transform of the function $\widetilde{\Omega}\left[\Psi_{\mu}, E / F, \psi: a\right]$ is, up to a positive constant, the function $\widetilde{\Omega}\left[\Psi_{\tilde{\mu}}, E / F, \bar{\psi}: a\right]$. Thus the function $\widetilde{\Omega}\left[\Psi_{\mu}, E / F, \psi: a\right]$ belongs to the space $\mathcal{F}(2 \mu)$. By Proposition 4 there exist unique constants $\xi_{\mu}^{\lambda}, \lambda \preceq 2 \mu$ such that

$$
\widetilde{\Omega}\left[\Psi_{\mu}, E / F, \psi: a\right]=\sum_{\lambda} \xi_{\mu}^{\lambda} \delta_{F}^{1 / 2}\left(\varpi^{2 \mu-\lambda}\right) \widetilde{\Omega}\left[\Phi_{\lambda}, \psi: a\right]
$$

or, equivalently,

$$
\Psi_{\mu} \leftrightarrow \sum_{\lambda} \xi_{\mu}^{\lambda} \delta_{F}^{1 / 2}\left(\varpi^{2 \mu-\lambda}\right) \Phi_{\lambda} .
$$

Motivated by (27), we define a linear map

$$
\beta: \mathcal{H}_{E} \rightarrow \mathcal{H}_{F}
$$

by

$$
\beta\left(f_{\mu}^{E}\right)=\sum_{\lambda \preceq 2 \mu} \xi_{\mu}^{\lambda} \delta_{F}^{1 / 2}\left(\varpi^{\lambda-2 \mu}\right) f_{\lambda}^{F} .
$$

We denote by $\beta_{\mathcal{S}}$ the corresponding linear transformation of the algebra $\mathbb{S}_{n}$ of symmetric polynomials to itself, that is,

$$
\mathcal{S}(\beta(f))=\beta_{\mathcal{S}}(\mathcal{S}(f))
$$

Since

$$
\mathcal{S}\left(f_{\lambda}^{F}\right)=\delta_{F}^{-1 / 2}\left(\varpi^{\lambda}\right) \sigma_{\lambda}, \quad \mathcal{S}\left(f_{\mu}^{E}\right)=\delta_{E}^{-1 / 2}\left(\varpi^{\mu}\right) \sigma_{\mu}
$$

we have

$$
\beta_{\mathcal{S}}\left(\sigma_{\mu}\right)=\sum_{\lambda \preceq 2 \mu} \xi_{\mu}^{\lambda} \sigma_{\lambda} .
$$

If we consider similarly the map $b_{\mathcal{S}}$ then

$$
b_{\mathcal{S}}(\sigma)(x)=\sigma_{\mu}\left(x^{2}\right)=\sum \theta_{\mu}^{\lambda} \sigma_{\lambda}(x) .
$$

Our definition amounts to saying that the function

$$
X \mapsto \int \Psi_{0}\left[{ }^{t} \bar{x} X x\right] f_{\lambda}^{E}(x) d x
$$

ANNALES SCIENTIFIQUES DE L'ÉCOLE NORMALE SUPÉRIEURE 
matches the function

$$
X \mapsto \int \Phi_{0}[X x] \beta\left(f_{\lambda}^{E}\right)(x) d x .
$$

By linearity, for any $f \in \mathcal{H}_{E}$, the function

$$
X \mapsto \int \Psi_{0}\left[{ }^{t} \bar{x} X x\right] f(x) d x
$$

matches the function

$$
X \mapsto \int \Phi_{0}[X x] \beta(f)(x) d x .
$$

Our main result is then equivalent to the assertion that $\beta=b$ or, what amounts to the same,

$$
\forall \mu, \forall \lambda \preceq 2 \mu, \quad \xi_{\mu}^{\lambda}=\theta_{\mu}^{\lambda} .
$$

We first list the properties of $\beta$ which follow from the definitions.

We have proved that $\Psi_{0} \leftrightarrow c \Phi_{0}$ where $c$ is a constant. Using the reduction formula we see that the orbital integrals of $\Psi_{0}$ and $\Phi_{0}$ agree on $A \cap K_{F}$. Thus $\Psi_{0} \leftrightarrow \Phi_{0}$, that is, the image of the unit element of $\mathcal{H}_{E}$ under $\beta$ is the unit element of $\mathcal{H}_{F}$.

We have $\sigma_{\tilde{\mu}}(x)=\sigma_{\mu}\left(x^{-1}\right)$. Also from the properties of the Kloosterman transform we have $\xi_{\mu}^{\lambda}=\xi_{\tilde{\mu}}^{\tilde{\lambda}}$. If $P \in \mathbb{S}_{n}$ is a symmetric polynomial, let us set $\check{P}(x)=P\left(x^{-1}\right)$. Clearly $\beta_{\mathcal{S}}(\check{P})=$ $\beta_{\mathcal{S}}(P)^{\check{r}}$. In addition $\mathcal{S}(\check{f})=\mathcal{S}(f)$. We conclude that

$$
\beta(f)^{\swarrow}=\beta(\check{f}) .
$$

Next, let us set

$$
\mu_{0}=(\underbrace{1,1, \ldots, 1}_{n}) .
$$

We have

$$
\sigma_{\lambda+\mu_{0}}(x)=\sigma_{\lambda}(x) x_{1} x_{2} \ldots x_{n}
$$

On the other hand

$$
\begin{aligned}
\Omega\left[\Psi_{\mu+\mu_{0}}: a\right] & =\Omega\left[\Psi_{\mu}: a \varpi^{2 \mu_{0}}\right] \\
& =\gamma\left(a \varpi^{2 \mu_{0}}\right) \sum_{\lambda} \xi_{\mu}^{\lambda} \delta_{F}^{1 / 2}\left(\varpi^{2 \mu-\lambda}\right) \Omega\left[\Phi_{\lambda}: a \varpi^{2 \mu_{0}}\right] \\
& =\gamma(a) \sum_{\lambda} \xi_{\mu}^{\lambda} \delta_{F}^{1 / 2}\left(\varpi^{2 \mu-\lambda-2 \mu_{0}}\right) \Omega\left[\Phi_{\lambda+2 \mu_{0}}: a\right] .
\end{aligned}
$$

It follows that

$$
\xi_{\mu+\mu_{0}}^{\lambda+2 \mu_{0}}=\xi_{\mu}^{\lambda}
$$

and

$$
\beta_{\mathcal{S}}\left(\sigma_{\lambda+\mu_{0}}\right)(x)=\beta_{\mathcal{S}}\left(\sigma_{\lambda}\right)(x)\left(x_{1} x_{2} \cdots x_{n}\right)^{2} .
$$

In particular, since $b_{\mathcal{S}}\left(\sigma_{0}\right)=\beta_{\mathcal{S}}\left(\sigma_{0}\right)$ we have also $b_{\mathcal{S}}\left(\sigma_{\mu_{0}}\right)=\beta_{\mathcal{S}}\left(\sigma_{\mu_{0}}\right)$. More generally

$$
b_{\mathcal{S}}\left(\sigma_{\mu}\right)=\beta_{\mathcal{S}}\left(\sigma_{\mu}\right)
$$

$4^{\mathrm{e}}$ SÉRIE - TOME $38-2005-\mathrm{N}^{\circ} 4$ 
if $\mu_{1}-\mu_{n}=0$.

The function $\sigma_{\lambda}$ is homogeneous of degree $|\lambda|$. Thus it is clear that if $P$ is homogeneous of degree $m$ then $\beta_{\mathcal{S}}(P)$ is homogeneous of degree $2 m$. Equivalently, if $f \in \mathcal{H}_{E}$ is supported on the set

$$
\left\{g \in G L(n, E):|\operatorname{det} g|_{E}=\left|\varpi^{m}\right|_{E}\right\}
$$

then $\beta(f)$ is supported on the set

$$
\left\{g \in G L(n, F):|\operatorname{det} g|_{F}=\left|\varpi^{2 m}\right|_{F}\right\} .
$$

By the same argument we used for $b$, it follows that for any Hecke function $f \in \mathcal{H}_{E}$ the function

$$
\left.X \mapsto \int \Psi^{1}{ }^{t} \bar{x} X x\right] f(x) d x
$$

matches the function

$$
X \mapsto \int \Phi^{1}[X x] \beta(f)(x) d x .
$$

Now the functions $\sigma_{\mu}$ with $\mu_{1}-\mu_{n}=0$ together with the functions $\sigma_{\lambda}$ with $\lambda_{1}-\lambda_{n}$ generate the algebra $\mathbb{S}_{n}$. The following proposition shows that $\beta_{\mathcal{S}}$ and $b_{\mathcal{S}}$ agree on a set of generators.

Proposition 5. - Suppose $\mu$ is such that $\mu_{1}-\mu_{n}=1$. Then, for all $\lambda$,

$$
\xi_{\mu}^{\lambda}=\theta_{\mu}^{\lambda}
$$

Proof. - Again our assertion is vacuous if $n=1$. Thus we may assume $n>1$ and our assertion true for $n-1$. By homogeneity we may as well assume that

$$
\mu=(\overbrace{r}^{\overbrace{1,1, \ldots, 1}^{n}, 0,0, \ldots, 0}) .
$$

If $\mu$ has the above form then any $\lambda \preceq 2 \mu$ has the form

$$
\lambda=(\overbrace{s}^{\overbrace{2,2, \ldots, 2}, \underbrace{1,1, \ldots, 1}_{2 t}, 0,0, \ldots 0})
$$

with

$$
0 \leqslant t \leqslant r, \quad s=r-t, \quad t \leqslant n-r .
$$

LEMMA 18. - With the above notation

$$
\theta_{\mu}^{\lambda}=(-1)^{t}
$$

Proof. - Replacing $\mu$ by $\tilde{\mu}$ and using homogeneity we may assume $2 r \leqslant n$. Then the inequality $t \leqslant n-r$ is superfluous. We first recall a property of the Schur functions. Given $\mu \in \mathcal{P}_{n}$ we say that a $\lambda \in \mathcal{P}_{n-1}$ interlaces $\mu$ and we write $\lambda \triangleleft \mu$ if

$$
\mu_{1} \geqslant \lambda_{1} \geqslant \mu_{2} \geqslant \lambda_{2} \geqslant \cdots \geqslant \mu_{n-1} \geqslant \lambda_{n-1} \geqslant \mu_{n} .
$$


Suppose $x=(y, 1), y=\left(y_{1}, y_{2}, \ldots, y_{n-1}\right)$. Then we have the branching formula.

$$
\sigma_{\mu}(x)=\sum_{\lambda \triangleleft \mu} \sigma_{\lambda}(y)
$$

This follows for instance from the fact that $\sigma_{\lambda}$ can be viewed as the character of an irreducible representation $\pi_{\mu}$ of $G L(n, \mathbb{C})$. The formula amounts to saying that the restriction of $\pi_{\mu}$ to $G L(n-1, \mathbb{C})$ is the direct sum of the representations $\pi_{\lambda}$ with $\lambda \triangleleft \mu$ (branching rule, see for instance [4, Theorem 8.11]).

The lemma is a tautology for $n=1$. Thus we may assume that $n>1$ and our assertion established for $n-1$. Consider the formula defining the constants $\theta_{\mu}^{\lambda}$.

$$
\sigma_{\mu}\left(x^{2}\right)=\sum_{\lambda \preceq 2 \mu} \theta_{\mu}^{\lambda} \sigma_{\lambda}(x) .
$$

We may assume that $x=(y, 1)$ because the resulting formula still defines the constants uniquely.

Suppose first that $r<n-r$. Then if $\lambda \preceq 2 \mu$ we have $s+2 t \leqslant 2 s+2 t=2 r<n$. In other words $\lambda$ has at least one trailing 0 . Using the branching formula for our $\mu$, we can write the left-hand side of (59) as

$$
\sigma_{\mu}\left(x^{2}\right)=\sigma_{\mu^{1}}\left(y^{2}\right)+\sigma_{\mu^{2}}\left(y^{2}\right)
$$

where

$$
\mu^{1}=(\overbrace{\underbrace{1,1, \ldots, 1}_{r}, 0,0, \ldots, 0}^{n-1})
$$

and

$$
\mu^{2}=(\overbrace{\underbrace{1,1, \ldots, 1}_{r-1}, 0,0, \ldots, 0}^{n-1}) .
$$

We can apply the induction hypothesis. We have

$$
\sigma_{\mu^{1}}\left(y^{2}\right)=\sum_{\lambda^{1} \preceq 2 \mu^{1}}(-1)^{t} \sigma_{\lambda^{1}}(y)
$$

where

$$
\lambda^{1}=\lambda_{t}^{1}=(\overbrace{\underbrace{2,2, \ldots, 2}_{s_{1}}}^{1,1,1, \ldots, 1}, 0,0, \ldots 0)
$$

with

$$
0 \leqslant t \leqslant r, \quad s=r-t .
$$

We stress that $\left|\lambda^{1}\right|=2 r$. On the other hand,

$$
\sigma_{\mu^{2}}\left(y^{2}\right)=\sum_{\lambda^{2} \preceq 2 \mu^{2}} \theta_{\mu^{2}}^{\lambda^{2}} \sigma_{\lambda^{2}}(y)
$$

$4^{\text {e }}$ SÉRIE - TOME $38-2005-\mathrm{N}^{\circ} 4$ 
with

$$
\lambda^{2}=\lambda_{t_{2}}^{2}=(\overbrace{\underbrace{, 2, \ldots, 2}_{s_{2}},}^{\underbrace{1,1, \ldots, 1}_{2 t_{2}}, 0,0, \ldots 0})
$$

with

$$
0 \leqslant t_{2} \leqslant r-1, \quad s_{2}=r-1-t_{2} .
$$

We stress that such a $\lambda^{2}$ verifies $\left|\lambda^{2}\right|=2 r-2$. On the right-hand side of (59) we can write

$$
\sum_{\lambda \preceq 2 \mu} \theta_{\mu}^{\lambda} \sum_{\lambda^{\prime} \triangleleft \lambda} \sigma_{\lambda^{\prime}}(y)
$$

For each $\lambda \preceq 2 \mu$ there is a unique $\lambda^{0} \triangleleft \lambda$ with $\left|\lambda^{0}\right|=|\lambda|=2 r$. If

$$
\lambda=\lambda_{t}=(\overbrace{\underbrace{2,2, \ldots, 2}_{s}}^{1,1, \ldots, 1}, 0,0, \ldots 0)
$$

where $0 \leqslant t \leqslant r, s=r-t$, it is

$$
\lambda_{t}^{0}=(\overbrace{\underbrace{2,2, \ldots, 2}_{s}}^{\underbrace{1,1, \ldots, 1}_{2 t}}, 0,0, \ldots 0) .
$$

In other words $\lambda_{t}^{0}$ is obtained by dropping the last trailing 0 from $\lambda$. Comparing terms $\sigma_{\nu}(y)$ with $|\nu|=2 r$ we get

$$
\sum_{0 \leqslant t \leqslant r}(-1)^{t} \sigma_{\lambda_{t}^{1}}(y)=\sum_{0 \leqslant t \leqslant r} \theta_{\mu}^{\lambda_{t}} \sigma_{\lambda_{t}^{0}}(y) .
$$

Comparing the coefficients in this formula we conclude that $\theta_{\mu}^{\lambda_{t}}=(-1)^{t}$ as was claimed.

Now suppose $r=n-r$. Thus

$$
\mu=(\underbrace{1,1, \ldots, 1}_{r}, \underbrace{0,0, \ldots, 0}_{r}) .
$$

The $\lambda$ such that $\lambda \preceq 2 \mu$ are of two types, those $\lambda$ for which there are at least a trailing 0 and one single element

$$
\lambda^{p}=(\underbrace{1,1, \ldots, 1}_{n}) .
$$

The $\lambda$ with a trailing 0 have the form

$$
\lambda=\lambda_{t}=(\overbrace{2,2, \ldots, 2}^{\underbrace{1,1, \ldots, 1}_{s}, 0,0, \ldots 0})
$$

with

$$
0 \leqslant t<r, \quad s=r-t
$$


We have

$$
\sigma_{\mu}\left(x^{2}\right)=\sum_{0 \leqslant t \leqslant r-1} \theta_{\mu}^{\lambda_{t}} \sigma_{\lambda_{t}}(x)+\theta_{\mu}^{\lambda^{p}} \sigma_{\lambda^{p}}(x) .
$$

Again we set $x=(y, 1)$ and we use the branching formula and the induction hypothesis. Here the only element which interlaces $\lambda^{p}$ is

$$
\lambda^{p 0}=(\underbrace{1,1, \ldots, 1}_{n-1}) .
$$

Then

$$
\sigma_{\lambda^{p}}(x)=\sigma_{\lambda^{p 0}}(y)
$$

and $\left|\lambda^{p 0}\right|=n-1=2 r-1$. When we compare terms $\sigma_{\nu}(y)$ with $|\nu|=2 r$ this term does not give a contribution. Thus we have again $\theta_{\mu}^{\lambda_{t}}=(-1)^{t}$. Thus we may write

$$
\sigma_{\mu}\left(x^{2}\right)=\sum(-1)^{t} \sigma_{\lambda}(x)+\theta_{\mu}^{\lambda^{p}} \sigma_{\lambda^{p}}(x) .
$$

where

To compute the remaining constant we apply the same approach as before, but this time we look for terms containing $\sigma_{\lambda^{p 0}}(y)$. Note that $\left|\lambda^{p 0}\right|=n-1=2 r-1$. Consider the left-hand side of (60). It can be written as before

$$
\sigma_{\mu^{1}}\left(y^{2}\right)+\sigma_{\mu^{2}}\left(y^{2}\right)
$$

In applying the induction hypothesis to $\sigma_{\mu^{1}}\left(y^{2}\right)$ we find terms $\sigma_{\nu}(y)$ with $|\nu|=2 r$. Thus we cannot find the term $\sigma_{\lambda^{p 0}}(y)$. In applying the induction hypothesis to $\sigma_{\mu^{2}}\left(y^{2}\right)$ we find terms containing $\sigma_{\nu}$ with $|\nu|=2 r-2$. Thus we cannot find the term $\sigma_{\lambda^{p 0}}(y)$ on the left-hand side. We apply formula (58) to the right-hand side of (60). We remark that there are only two $\lambda$ such that $\lambda^{p 0} \triangleleft \lambda$, namely $\lambda^{p}$ and

$$
\lambda^{p p}:=(2, \underbrace{1,1, \ldots, 1}_{2 r-2}, 0) .
$$

Thus we find

$$
(-1)^{r-1} \sigma_{\lambda^{p 0}}(y)+\theta^{\lambda^{p}} \sigma_{\lambda^{p 0}}(y)=0
$$

or

$$
\theta^{\lambda^{p}}=(-1)^{r}
$$

This concludes the proof of the lemma.

It remains to prove that $\xi_{\mu}^{\lambda}=\theta_{\mu}^{\lambda}$. Replacing $\mu$ by $\tilde{\mu}$ and using homogeneity we may assume $2 r \leqslant n$.

We pass to the computation of the constants $\xi_{\mu}^{\lambda}$. We first compute the coefficient $\xi_{\mu}^{\lambda_{p}}$ in the case $2 r=n$. We state this as a lemma.

LEMmA 19. - Assume $2 r=n$. Then, with the above notation,

$$
\xi_{\mu}^{\lambda_{p}}=(-1)^{r} .
$$

$4^{\mathrm{e}}$ SÉRIE - TOME $38-2005-\mathrm{N}^{\circ} 4$ 
Proof. - The matching (54) implies an equality of the corresponding orbital integrals of the functions on the left and right-hand sides, up to the transfer factor. We consider the orbital integrals for the matrix $w_{n} z$ where $z \in F^{\times}$. We find

$$
\Omega\left[\Psi_{\mu}, E / F, \psi: w_{n} z\right]=\gamma\left(w_{n} z, \psi\right) \sum_{\lambda \preceq 2 \mu} \xi_{\mu}^{\lambda} \delta_{F}^{1 / 2}\left(\varpi^{2 \mu-\lambda}\right) \Omega\left[\Phi_{\lambda}, \psi: w_{n} z\right] .
$$

It is immediately verified that for any $\lambda$ of the form $\lambda=(*, *, \ldots, *, 0)$, the integral $\Omega\left[\Phi_{\lambda}: w_{n} z\right]$ vanishes unless $|z|=1$. We take $|z|=\left|\varpi^{-1}\right|$. These integrals vanish then. On the other hand we have then $\Omega\left[\Phi_{\lambda^{p}}: w_{n} z\right]=1$. We claim that

$$
\Omega\left[\Psi_{\mu}: w_{n} z\right]=q^{-r^{2}}
$$

We illustrate the case $n=4$ but the computation is general.

$$
\begin{aligned}
& \Omega\left[\Psi_{\mu}: w_{4} z\right] \\
& \quad=\int \Psi_{0}\left[\begin{array}{cccc}
0 & 0 & 0 & z \varpi \\
0 & 0 & z \varpi & z \varpi x_{1} \\
0 & z \varpi & z x_{2} & z u \\
z \varpi & z \varpi \bar{x}_{1} & z \bar{u} & z w
\end{array}\right] \psi\left(x_{1}+\bar{x}_{1}\right) \psi\left(x_{2}\right) d x_{1} d x_{2} d u d w .
\end{aligned}
$$

Here $x_{1}, u$ are in $E$ and $x_{2}, w$ are in $F$. After integrating we find the stated result. On the other hand, the transfer factor $\gamma\left(w_{n} z, \psi\right)$, viewed as a function of $n$, has period 8 for an arbitrary quadratic extension (see [6, (8)]). Here the extension is unramified so that the Weil constant $c(E / F, \psi)$ is 1 . Thus the factor is in fact periodic of period 4 . Its first 4 values (starting with $n=1)$ are

$$
1, \quad \eta(z), \quad \eta(z), \quad 1 .
$$

Thus here $\gamma\left(w_{n} z, \psi\right)=(-1)^{r}$. It follows that $\xi_{\mu}^{\lambda^{p}}=(-1)^{r}$.

It remains to compute the remaining coefficients $\xi_{\mu}^{\lambda}$. Now consider the formula which defines the constants $\xi_{\mu}^{\lambda}$.

$$
\Omega\left[\Psi_{\mu}: a\right]=\gamma(a) \sum_{\lambda \preceq 2 \mu} \xi_{\mu}^{\lambda} \delta_{F}^{1 / 2}\left(\varpi^{2 \mu-\lambda}\right) \Omega\left[\Phi_{\lambda}: a\right] .
$$

Let us set

$$
a=\left(a_{1}, a^{\prime}\right), \quad\left|a_{1}\right|=\left|\varpi^{-2}\right| .
$$

and apply the reduction formulas. If $2 r=n$ the term corresponding to $\lambda^{p}$ on the right is then 0 . Thus in all cases the non-zero terms on the right correspond to $\lambda$ 's of the following form. Let us set

$$
\mu=\left(1, \mu^{\prime}\right)
$$

Then

$$
\lambda=\left(2, \lambda^{\prime}\right), \quad \lambda^{\prime} \preceq 2 \mu^{\prime} .
$$

We get

$$
q^{-2(n-1) \mu_{1}+2\left|\mu^{\prime}\right|} \Omega\left[\Psi_{\mu^{\prime}}: a^{\prime}\right]=\gamma\left(a^{\prime}\right) \sum q^{-(n-1) \lambda_{1}+\left|\lambda^{\prime}\right|} \xi_{\mu}^{\lambda} \delta_{F}^{1 / 2}\left(\varpi^{2 \mu-\lambda}\right) \Omega\left[\Phi_{\lambda^{\prime}}: a^{\prime}\right] .
$$


After simplification we get

$$
\Omega\left[\Psi_{\mu^{\prime}}: a^{\prime}\right]=\gamma\left(a^{\prime}\right) \sum_{\lambda^{\prime} \preceq 2 \mu^{\prime}} \Omega\left[\Phi_{\lambda^{\prime}}: a^{\prime}\right] \xi_{\mu}^{\lambda} \delta_{F}^{1 / 2}\left(\varpi^{2 \mu-\lambda}\right) .
$$

In addition $\delta_{F}^{1 / 2}\left(\varpi^{2 \mu-\lambda}\right)=\delta_{F}^{1 / 2}\left(\varpi^{2 \mu^{\prime}-\lambda^{\prime}}\right)$ since $\operatorname{det}\left(\varpi^{2 \mu^{\prime}-\lambda^{\prime}}\right)=1$. It follows that $\xi_{\mu}^{\lambda}=\xi_{\mu^{\prime}}^{\lambda^{\prime}}$. On the other hand from Lemma 18 we have $\theta_{\mu}^{\lambda}=\theta_{\mu^{\prime}}^{\lambda^{\prime}}$. Applying the induction hypothesis we get $\xi_{\mu}^{\lambda}=\theta_{\mu}^{\lambda}$ as was claimed. This concludes the proof of Proposition 5.

We have proved that $\beta_{\mathcal{S}}$ and $b_{\mathcal{S}}$ agree on a set of generators of $\operatorname{Spec}\left[\mathbb{S}_{n}\right]$ as an algebra. To show that $\beta=b$ it will suffice to show that $\beta_{\mathcal{S}}$ is a homomorphism of algebras. This is equivalent to saying that for any $x \in \operatorname{Spec}\left[\mathbb{S}_{n}\right]$, the map

$$
P \mapsto \beta_{\mathcal{S}}(P)(x)
$$

is a character of the algebra $\mathbb{S}_{n}$. Obviously it suffices to prove it for $x$ in a Zariski dense subset. Thus we see that our assertion that $\beta=b$, or equivalently, the main theorem, will be established if we prove that,

$$
f \mapsto \mathcal{S}(\beta(f))(x)
$$

is a character for all $x$ in a Zariski dense subset. This will be taken up in the next section.

\section{A simple trace formula}

In this section we consider again a quadratic extension of number fields $E / F$. We assume that every real place of $F$ splits in $E$. We consider a cuspidal automorphic representation $\pi^{0}$ of $G L\left(n, F_{\mathbb{A}}\right)$. We assume that at a fixed split place $v_{\text {cusp }}$ the local component of $\pi^{0}$ is supercuspidal. We let $S$ be a finite set of places of $F$ containing the places at infinity, the even places, the places $v$ where the local component $\pi_{v}^{0}$ is ramified, all the places of $F$ inert and ramified in $E$, all the finite places $v$ where the character $\psi_{v}$ has a conductor different from $\mathcal{O}_{v}$. In particular $S$ contains $v_{\text {cusp. }}$. For $s \in S$ we take $\Phi_{v}$ so that

$$
\mathcal{B}_{\pi_{v}^{0}}\left(\Phi_{v}\right) \neq 0 \text {. }
$$

We take, as we may, the function $\Phi_{v_{\text {cusp }}}$ to be a supercuspidal function. At each inert place $v \notin S$ we have the linear map $\beta_{v}$ :

$$
\beta_{v}: \mathcal{H}_{w} \rightarrow \mathcal{H}_{v},
$$

where $w$ is the place above $v$. Recall that $\beta_{v}$ takes the unit element to the unit element. Moreover, $\beta_{v}$ is so defined that it satisfies the matching assertion of the main theorem.

We fix a place $v_{\text {rel }}$ inert and not in $S$. We denote by $w_{\text {rel }}$ the corresponding place of $E$. Our goal in this section is to prove the following proposition.

PROPOSITION 6. - The map

$$
f \mapsto \mathcal{S}\left(\beta_{v_{\mathrm{rel}}}(f)\right)\left(\pi_{v_{\mathrm{rel}}}^{0}\right)
$$

is a character of $\mathcal{H}_{w_{\mathrm{rel}}}$.

$4^{\mathrm{e}}$ SÉRIE - TOME $38-2005-\mathrm{N}^{\circ} 4$ 
Proof. - We follow step by step the construction of Section 3. Simply we replace at each inert place $v \notin S$ the base change map $b_{v}$ by $\beta_{v}$. The set of places $T_{1}$ may be chosen not to contain $v_{\text {rel }}$. It is convenient to have the set $T_{2}$ not to contain $v_{\text {rel }}$. For that purpose we appeal to a lemma.

LEMMA 20. - One can choose the representatives $\xi$ in such a way that $\xi_{v_{\mathrm{rel}}}$ is in $K_{w_{\mathrm{rel}}}$.

Proof. - Recall that the only $\xi$ which appear have $\operatorname{det} \xi$ is a norm in the extension $E_{w_{\mathrm{rel}}} / F_{v_{\mathrm{re}}}$. At any rate there is $g \in G L\left(n, E_{w_{\text {rel }}}\right)$ such that

$$
\xi={ }^{t} \bar{g} g
$$

Thus there is a sequence $\gamma_{i} \in G L(n, E)$ such that

$$
\lim ^{t} \bar{\gamma}_{i} \xi \gamma_{i}=1
$$

where the limit is in the space $S\left(F_{v_{\text {rel }}}\right)$. Since $K_{w_{\text {rel }}} \cap S\left(F_{v_{\text {rel }}}\right)$ is open, we have

$$
{ }^{t} \bar{\gamma}_{i} \xi \gamma_{i} \in K_{w_{\text {rel }}} \cap S\left(F_{v_{\text {rel }}}\right)
$$

for some $i$.

Since $\Phi_{v_{\text {cusp }}}$ is a supercuspidal function the function $f_{v_{\text {cusp }, 1, v_{\text {cusp }, 2}}^{\xi}}$ can be taken to be supercuspidal, as function of the second variable.

Because of the supercuspidality condition the expression (12) is equal to the sum

$$
\sum_{\xi \in \Xi} \sum_{\Pi} \mathcal{R}_{\Pi}^{\xi}\left(f^{\xi}\right)
$$

where the sum is over all cuspidal automorphic representations $\Pi$ with a supercuspidal component at $v_{\text {cusp }, 2}$ (and hence at $v_{\text {cusp }, 1}$ ). Likewise, the expression (14) is equal to

$$
\sum_{\pi} \mathcal{B}_{\pi}(\Phi)
$$

where the sum is over all cuspidal automorphic representations $\pi$ with a supercuspidal component at $v_{\text {cusp }}$.

At this point we have the analogue of (21):

$$
\sum_{\Pi_{\infty}=\pi_{\infty}^{0} \otimes \pi_{\infty}^{0}} \sum_{\xi \in \Xi} \mathcal{R}_{\Pi}^{\xi}\left(f^{\xi}\right)=c \mathcal{B}_{\pi_{\infty}^{0}}\left(\Phi_{\infty}\right) \prod_{v \notin S_{2}} \mathcal{S}\left(\Phi_{v}\right)\left(\pi_{v}^{0}\right)
$$

The sum on the left is finite. We remind ourselves of the relation between the functions. Let $U_{2}$ be the set of places of $E$ above a place in $S_{2}$. Each function $f^{\xi}$ is a tensor product. The factors $f_{u}^{\xi}$ corresponding to a finite place in $u \in U_{2}$ are fixed. All the factors $f_{u}^{\xi}$ corresponding to a place $u \notin U_{2}$ are equal to an arbitrary Hecke function $f_{u}$. If $v \notin S_{2}$ splits into $v_{1}, v_{2}$ then $\Phi_{v}$ is the base change of $f_{v_{1}} \otimes f_{v_{2}}$, that is, the convolution $f_{v_{1}} * f_{v_{2}}$. If $v \notin S_{2}$ is inert and $w \notin U_{2}$ is the corresponding place of $E$ then $\Phi_{v}=\beta_{v}\left(f_{w}\right)$. We fix a function $\Phi_{\infty}$ such that

$$
\mathcal{B}_{\pi_{\infty}^{0}}\left(\Phi_{\infty}\right) \neq 0 .
$$

We fix then correspondingly $\Psi_{\infty}$ and the functions $f_{\infty}^{\xi}$. 
We label the representations on the left-hand side $\Pi^{\gamma}, 0 \leqslant \gamma \leqslant s$. We set

$$
C^{\gamma}=\sum_{\xi \in \Xi} \mathcal{R}_{\Pi^{\gamma}}^{\xi}\left(f_{U_{2}}^{\xi} \otimes \mathbf{1}^{U_{2}}\right)
$$

Then the left-hand side takes the form

$$
\sum_{0 \leqslant \gamma \leqslant s} C^{\gamma} \prod_{u \notin U_{2}} \mathcal{S}\left(f_{u}\right)\left(\Pi_{u}^{\gamma}\right)
$$

If we choose $\Phi_{v}=\Phi_{v}^{1}$ for all $v \notin S_{2}$ we obtain on the right of Equation (61) a non-zero result namely

$$
c \mathcal{B}_{\pi_{\infty}^{0}}\left(\Phi_{\infty}\right) .
$$

Thus there is a least one representation $\Pi^{0}$ such that $C^{0} \neq 0$

$$
C^{0}:=\sum_{\xi \in \Xi} \mathcal{R}_{\Pi^{0}}^{\xi}\left(f^{\xi}\right), \quad f_{u}=f_{u}^{1}, \quad u \notin U_{2} .
$$

Then $C^{0} \neq 0$. We can choose a finite set of places $T_{3}=\left\{u_{\gamma} \mid 1 \leqslant \gamma \leqslant s\right\}$ not containing $w_{\text {rel }}$ such that

$$
\Pi_{u_{\gamma}}^{0} \not \Pi_{u_{\gamma}}^{\gamma} \text {. }
$$

As before, we use strong multiplicity one. We choose then Hecke functions $f_{u_{\gamma}}$ such that

$$
\forall \gamma, \mathcal{S}\left(f_{u_{\gamma}}\right)\left(\Pi_{u_{\gamma}}^{0}\right)=1, \quad \mathcal{S}\left(f_{u_{\gamma}}\right)\left(\Pi_{u_{\gamma}}^{\gamma}\right)=0 .
$$

If $u_{\gamma}$ is above an inert place of $F$ we denote this place by $v_{\gamma}$. If $u_{\gamma}$ is above a place $v_{\gamma}$ which splits into $u_{\gamma}, u_{\gamma}^{\prime}$ then $\Pi_{u_{\gamma}}^{\gamma} \simeq \Pi_{u_{\gamma}^{\prime}}^{\gamma}$. Thus we may as well assume that $u_{\gamma}^{\prime}$ is not in $T_{3}$. Now at all places $u \notin U_{2} \cup T_{3}$ and distinct from $w_{\text {rel }}$ we take $f_{u}=f_{u}^{1}$. Then the left-hand side of Equation (61) reduces to

$$
C^{0} \mathcal{S}\left(f_{w_{\mathrm{rel}}}\right)\left(\Pi_{w_{\mathrm{rel}}}^{0}\right)
$$

The right-hand side of Equation (61) is then the product of the following factors

$$
\begin{aligned}
& \mathcal{S}\left(\beta_{v_{\text {rel }}}\left(f_{w_{\text {rel }}}\right)\right)\left(\pi_{v_{\text {rel }}}^{0}\right), \\
& c \mathcal{B}_{\pi_{\infty}}\left(f_{\infty}\right), \\
& \prod_{v_{\gamma} \text { split }} S\left(f_{u_{\gamma}}\right)\left(\pi_{v_{\gamma}}^{0}\right), \\
& \prod_{v_{\gamma} \text { inert }} S\left(\beta_{v_{\gamma}}\left(f_{u_{\gamma}}\right)\right)\left(\pi_{v_{\gamma}}^{0}\right) .
\end{aligned}
$$

Since $C^{0} \neq 0$ we see that the three last factors are non-zero and we conclude that

$$
\mathcal{S}\left(\beta_{v_{\text {rel }}}\left(f_{w_{\text {rel }}}\right)\right)\left(\pi_{v_{\text {rel }}}^{0}\right)=c_{1} \mathcal{S}\left(f_{w_{\text {rel }}}\right)\left(\Pi_{w_{\text {rel }}}^{0}\right) .
$$

By taking $f_{w_{\text {rel }}}$ to be the unit element we see that $c_{1}=1$. This concludes the proof of the proposition.

$4^{\mathrm{e}}$ SÉRIE - TOME $38-2005-\mathrm{N}^{\circ} 4$ 


\section{End of proof}

Now we let $E / F$ be an unramified quadratic extension of local fields. We can always write it as $E_{w_{\text {rel }}} / F_{v_{\text {rel }}}$ for a suitable quadratic extension of number fields of the type considered in the previous section. We can choose $\psi$ so that the conductor of $\psi_{v_{\text {rel }}}$ is $\mathcal{O}_{v_{\text {rel }}}$. Consider the representations $\pi^{0}$ of the type discussed in the previous section. The Langlands class $x \in \mathbb{C}^{\times n}$ of their components $\pi_{v_{\text {rel }}}^{0}$ is Zariski dense.

For the convenience of the reader we recall the proof. We have to prove that if $f_{v_{\text {rel }}}$ is a nonzero element of $\mathcal{H}_{v_{\text {rel }}}$ then there exists a $\pi^{0}$ of the above type such that $\pi_{v_{\text {rel }}}^{0}\left(f_{v_{\text {rel }}}\right) \neq 0$. To that end we set

$$
\begin{aligned}
& f_{v_{\text {rel }}}^{2}(g)=\overline{f_{v_{\text {rel }}(g)}} \\
& f_{v_{\text {rel }}}^{1}(x)=\int_{G_{v_{\text {rel }}}} f_{v_{\text {rel }}}^{2}(x g) f_{v_{\text {rel }}}(g) d g .
\end{aligned}
$$

Thus $f_{v_{\text {rel }}}^{1}(e) \neq 0$. For each finite place $v$ we choose a smooth function of compact support $f_{v}$ such that $f_{v}(e) \neq 0$. At the place $v_{\text {cusp }}$ we furthermore assume that $f_{v_{\text {cusp }}}$ is a cuspidal function. Of course, at almost all places, $f_{v}$ is the characteristic function of $K_{v}$. We let $f_{\infty}$ be a smooth function of compact support on $Z_{+} \backslash G_{\infty}$. We consider the following functions, where $i=1,2$,

$$
\begin{aligned}
f^{i}(g) & =f_{\infty}\left(g_{\infty}\right) f_{v_{\mathrm{rel}}}^{i}\left(g_{v_{\mathrm{rel}}}\right) \prod_{v \neq v_{\mathrm{rel}}} f_{v}\left(g_{v}\right), \\
\phi^{i}(g) & =\sum_{\gamma \in G(F)} f^{i}(\gamma g) .
\end{aligned}
$$

If we write $f^{1}(g)=f_{\infty}\left(g_{\infty}\right) f^{\infty}\left(g^{\infty}\right)$ we see that $f^{\infty}(\gamma) \neq 0$ implies that $\gamma$ is in a discrete subset $\Gamma$ of $G_{\infty}$ containing $e$. We choose $f_{\infty}$ so that $f_{\infty}(e) \neq 0$ and $f_{\infty}(\gamma) \neq 0, \gamma \in \Gamma$, imply $\gamma=e$. Thus $\phi^{1}(e) \neq 0$. The functions $\phi^{1}$ and $\phi^{2}$ belong to the closure of the space spanned by the cusp forms. Moreover $\phi^{1}(g)=\int \phi^{2}(g x) f_{v_{\text {rel }}}(x) d x$. Therefore there is at least one automorphic cuspidal representation $\pi^{0}$ such that the image of $\phi^{1}$ under the orthogonal projection $P$ on the space of $\pi^{0}$ is non-zero. We have

$$
P \phi^{1}(g)=\int\left(P \phi^{2}\right)(g x) f_{v_{\mathrm{rel}}}(x) d x
$$

that is,

$$
\pi_{v_{\mathrm{rel}}}^{0}\left(f_{v_{\mathrm{rel}}}\right) P \phi^{2}=P \phi^{1} .
$$

This implies that $\pi_{v_{\text {rel }}}^{0}\left(f_{v_{\text {rel }}}\right) \neq 0$ and we are done.

Thus the map

$$
f \mapsto \mathcal{S}(\beta(f))(x)
$$

is a character of $\mathcal{H}_{w_{\text {rel }}}$ for $x$ in a Zariski dense set. As we have seen this implies that $\beta=b$. 


\section{Three lemmas on Bessel distributions}

\subsection{Proof of Lemma 1}

We prove Lemma 1. For the proof of this lemma we drop the index infinity. We will need the positive type Bessel distribution

$$
\mathcal{P}_{\pi}(\Phi)=\sum \pi(\Phi) W_{i}(e) \bar{W}_{i}(e)
$$

Note that

$$
\mathcal{B}_{\pi}(\Phi)=\mathcal{P}_{\pi}\left(\Phi^{\prime}\right)
$$

where $\Phi^{\prime}(g)=\Phi\left(w_{n} g\right)$. Thus we can rewrite the assumption as

$$
\sum_{\pi} c(\pi) \mathcal{P}_{\pi}(\Phi)=0
$$

for all $\Phi$. By taking the functions $\Phi$ to be of positive type we see that we have

$$
\sum_{\pi} \Re c(\pi) \mathcal{P}_{\pi}(f)=0, \quad \sum_{\pi} \Im c(\pi) \mathcal{P}_{\pi}(f)=0
$$

Thus it suffices to prove the lemma when the coefficients are real. We derive a contradiction if not all terms are 0 . We can regroup together the terms with $c(\pi)>0$ and the terms with $c(\pi)<0$. After a change of notation, we have now an identity

$$
\sum_{\pi \in X} c(\pi) \mathcal{P}_{\pi}(f)=\sum_{\pi \in Y} c(\pi) \mathcal{P}_{\pi}(f),
$$

where $X$ and $Y$ are disjoint, in general, infinite sets and $c(\pi)>0$ for all $\pi$. We then derive a contradiction. At the cost of changing the Hilbert norm on $\pi$ we can rewrite this expression in the form

$$
\sum_{\pi \in X} \mathcal{P}_{\pi}(f)=\sum_{\pi \in Y} \mathcal{P}_{\pi}(f)
$$

Recall that to a distribution of positive type $\theta$ we can associate a unitary representation in the following way. Indeed, on the space $\mathcal{C}_{c}^{\infty}\left(G_{\infty}\right)$

$$
\left(f_{1}, f_{2}\right) \mapsto \theta\left(f_{2}^{*} * f_{1}\right)
$$

is a positive semi-definite Hermitian form, invariant under right shifts. Passing to the quotient we get a positive definite Hermitian form on a quotient of $\mathcal{C}_{c}^{\infty}\left(G_{\infty}\right)$. Then we complete the space for this norm. We obtain a Hilbert space on which $G_{\infty}$ acts. Applying this construction to $\mathcal{P}_{\pi}$ we recover $\pi$. In a more precise way, we can regard the Whittaker linear form $\lambda_{\pi}$ as a generalized vector (i.e. a vector which has scalar product with smooth vectors). Then

$$
\mathcal{P}_{\pi}\left(f^{*} * f\right)=\left(\pi(f) \lambda_{\pi}, \pi(f) \lambda_{\pi}\right) .
$$

Applying this construction to the two sides of our equality we recover the direct Hilbert sums

$$
\bigoplus_{\pi \in X} \pi, \quad \bigoplus_{\pi \in Y} \pi
$$

$4^{\text {e }}$ SÉRIE - TOME $38-2005-\mathrm{N}^{\circ} 4$ 
respectively. To see it is so consider the direct sum

$$
\mathcal{H}:=\bigoplus_{\pi \in X} \pi
$$

Recall $\mathcal{H}$ can be viewed as the space of $t$-uples $\left(v_{\pi}\right)_{\pi \in X}$ such that

$$
\sum_{\pi}\left(v_{\pi}, v_{\pi}\right)<+\infty
$$

Since

$$
\sum_{\pi \in X} \mathcal{P}_{\pi}\left(f^{*} * f\right)=\sum\left(\pi(f) \lambda_{\pi}, \pi(f) \lambda_{\pi}\right)<+\infty
$$

we can consider the closure $\mathcal{V}$ in $\mathcal{H}$ of the space spanned by $t$-uples

$$
\left(\pi(f) \lambda_{\pi}\right)_{\pi \in X}
$$

Since the projection of $\mathcal{V}$ on each $\pi$ is non-zero, we see that $\mathcal{V}$ is the Hilbert direct sum of the $\pi \in X$. On the other hand calling $\theta$ the sum of the $\mathcal{P}_{\pi}$ we have

$$
\theta\left(f^{*} * f\right)=\sum_{\pi \in X}\left(\pi(f) \lambda_{\pi}, \pi(f) \lambda_{\pi}\right) .
$$

Thus $\theta\left(f^{*} * f\right)=0$ if and only if $\pi(f) \lambda_{\pi}=0$ for all $\pi$. Our assertion follows. Thus we have

$$
\bigoplus_{\pi \in X} \pi=\bigoplus_{\pi \in Y} \pi
$$

Since $X \cap Y=\emptyset$ this is a contradiction.

\subsection{A formal lemma}

We now go back to the situation discussed before (20). Consider one of the distributions $\mathcal{R}_{\Pi}^{\xi}$. We write $f$ for the function $f^{\xi}$. Let $\infty_{F}$ be the set of infinite places of $F$. Each $v \in \infty_{F}$ splits into $v_{1}, v_{2}$ and $\Pi_{v_{1}} \simeq \Pi_{v_{2}}$. Call $\pi_{v}$ the equivalence class of this representation. The component $f_{v_{1}, v_{2}}$ of $f$ is arbitrary. We set

$$
\Phi_{v}(g)=\int_{G L\left(n, F_{v}\right)} f_{v_{1}, v_{2}}\left(\xi^{-1 t} h^{-1}, h g\right) .
$$

We can define the local Bessel distribution $\mathcal{B}_{\pi_{v}}$. On the other hand we have chosen a finite set $R$ of finite places of $E$ and for each $v \in R$ we have fixed the local component $f_{v}$. At the finite places $v \notin R$ the function $f_{v}$ is a Hecke function.

LEMMA 21. - With the above notations, there is a constant $c$ such that

$$
\mathcal{R}_{\Pi}^{\xi}(f)=c \prod_{v \in \infty_{F}} \mathcal{B}_{\pi_{v}}\left(\Phi_{v}\right) \prod_{v \text { finite } \notin R} \mathcal{S}\left(f_{v}\right)\left(\Pi_{v}\right) .
$$


Proof. -

$$
\mathcal{R}_{\Pi}^{\xi}(f)=\sum \mu\left(\Pi(f) \phi_{i}\right) \overline{\int \phi_{i}(u) \bar{\theta}(u \bar{u}) d u} .
$$

Here

$$
\mu(\phi)=\int_{H^{\xi}(F) \backslash H^{\xi}\left(F_{\mathrm{A}}\right)} \phi(h) d h .
$$

In view of our choice of functions, the functions $\phi_{i}$ belong to a sub vector space $V$ invariant under $\prod_{v \in \infty_{E}} G_{v}$. In turn $V$ is a finite direct sum of subspaces $V_{i}$ invariant and irreducible under $\prod_{v \in \infty_{E}} G_{v}$. The representation of $\prod_{v \in \infty_{E}} G_{v}$ on each $V_{i}$ is equivalent to

$$
\bigotimes_{v \in \infty_{F}} \Pi_{v_{1}} \otimes \Pi_{v_{2}}
$$

The restriction $\mu_{i}$ of $\mu$ to $V_{i}$ is a constant $c_{i}$ times a tensor product

$$
\bigotimes_{v \in \infty_{F}} \mu_{v}
$$

where $\mu_{v}$ is a linear form on $\pi_{v} \otimes \pi_{v}$ invariant under $H_{v}^{\xi}$. Likewise the restriction of the linear form

$$
\phi \mapsto \int \phi(u) \bar{\theta}(u \bar{u}) d u
$$

is a constant times a tensor product

$$
\bigotimes_{v \infty_{F}} \mu_{v, 1} \otimes \mu_{v, 1}
$$

where $\mu_{v, 1}$ is a linear form on the space of $\pi_{v}$ transforming under the character $\theta_{v}$. It follows that

$$
\mathcal{R}_{\Pi}^{\xi}(f)=c \prod_{v \in \infty_{F}} \mathcal{R}_{v}\left(f_{v_{1}, v_{2}}\right) \prod_{v \text { finite } \notin R} \mathcal{S}\left(f_{v}\right)\left(\Pi_{v}\right)
$$

where

$$
\mathcal{R}_{v}\left(f_{v_{1}, v_{2}}\right)=\sum \mu_{v}\left(\pi_{v} \otimes \pi_{v}\left(f_{v_{1}, v_{2}}\right) \phi_{i}\right) \overline{\mu_{v, 1} \otimes \mu_{v, 1}\left(\phi_{i}\right)}
$$

and $\phi_{i}$ is an orthonormal basis of $\pi_{v} \otimes \pi_{v}$.

To conclude we apply another formal lemma, valid for any local field (see [6, (7)]).

LEMMA 22.-Suppose that $F$ is a local field. Let $\pi$ be a unitary irreducible generic representation of $G L(n, F)$. Let $\lambda$ be a linear form on $\pi \otimes \pi$ invariant under the group $H^{\xi}(F)$. Let $\mu_{1}$ be a linear form on $\pi$ transforming under the character $\theta$. Consider the distributions

$$
\begin{aligned}
& \mathcal{R}(f)=\sum \lambda\left(\pi \otimes \pi(f) \phi_{i}\right) \overline{\mu_{1} \otimes \mu_{1}\left(\phi_{i}\right)}, \\
& \mathcal{B}(\Phi)=\sum \mu_{2}\left(\pi(\Phi) \phi_{i}\right) \overline{\mu_{1}\left(\phi_{i}\right)} .
\end{aligned}
$$

Then there is a constant $c$ such that

$$
\mathcal{R}(f)=c \mathcal{B}(\Phi)
$$

$4^{\mathrm{e}}$ SÉRIE - TOME $38-2005-\mathrm{N}^{\circ} 4$ 
where

$$
\Phi(g)=\int f\left(\xi^{-1 t} h^{-1}, h g\right) d h
$$

\subsection{A lemma of Shalika}

LEMMA 23. - Let $\pi$ be a unitary irreducible generic representation of $G L(n, \mathbb{R})$. The Bessel distribution $\mathcal{B}_{\pi}$ has a non-zero restriction to the open set

$$
{ }^{t} N(\mathbb{R}) A(\mathbb{R}) N(\mathbb{R})
$$

Proof. - We may replace $\mathcal{B}_{\pi}$ by the positive type Bessel distribution

$$
\mathcal{P}_{\pi}(f)=\sum \pi(f) W_{i}(e) \overline{W_{i}(e)}
$$

Our assertion is then that the restriction of $\mathcal{P}_{\pi}$ to the open set

$$
N(\mathbb{R}) w_{n} A(\mathbb{R}) N(\mathbb{R})
$$

is non-zero. Suppose it is. Then $\mathcal{P}_{\pi}$ is a distribution supported on the complement of that set. Moreover it is an eigenfunction of the Casimir operator and transforms on both sides under non-degenerate characters of $N_{n}(F)$. By Proposition 2.10 of [16] it follows that $\mathcal{P}_{\pi}=0$, a contradiction.

\section{Concluding remarks}

We briefly indicate how to obtain more precise global results. Assume $n$ is even. Let $\widetilde{H}^{\xi}$ be the similitude group corresponding to $H^{\xi}$ and $\lambda: \widetilde{H}^{\xi} \rightarrow F^{\times}$the similitude ratio. Let $\chi$ be an idele class character of $F$. We say that an automorphic representation $\Pi$ is distinguished by $\left(\widetilde{H}^{\xi}, \chi\right)$ if the central character of $\Pi$ is the character $z \mapsto \chi(z \bar{z})$ and the following integral is non-zero for at least one $\phi$ in the space of $\Pi$ :

$$
\int_{Z\left(E_{\mathrm{\AA}}\right) \widetilde{H}^{\xi}(F) \backslash \widetilde{H}^{\xi}\left(F_{\mathrm{\AA}}\right)} \phi(\tilde{h}) \chi^{-1}(\lambda(\tilde{h})) d \tilde{h} .
$$

PROPOSITION 7. - A representation $\Pi$ is the base change of a representation $\pi$ of central character $\omega$ if and only if it is distinguished by a pair $\left(\widetilde{H}^{\xi}, \omega \eta^{n / 2}\right)$.

Proof (Sketch). - We simply indicate which modifications must be made to the proof of Theorem 2. At a place $v$ of $F$ inert in $E$ we have

$$
\gamma_{v}(a z)=\gamma_{v}(a) \eta_{v}^{n / 2}(z)
$$

Thus globally if $\Psi \leftrightarrow \Phi$ then for each $z \in F_{\mathbb{A}}^{\times}$the function

$$
X \mapsto \Psi(X z)
$$

matches the function

$$
X \mapsto \eta(z)^{n / 2} \Phi(X z)
$$


For each $z \in F_{\mathbb{A}}^{\times}$we have

$$
\int K_{\Psi}(z u) \theta(u \bar{u}) d u=\eta^{n / 2}(z) \int K_{\Phi}\left(u_{1}, u_{2} z\right) \theta\left(u_{1}\right)^{-1} \theta\left(u_{2}\right) d u_{2} .
$$

Integrating over $z$ we get instead of (18)

$$
\begin{aligned}
& \int_{F_{\mathrm{A}}^{\times} \times N(E) \backslash N\left(E_{\mathrm{A}}\right)} K_{\Psi}(z u) \theta(u \bar{u}) \chi(z) d z d u \\
= & \int_{F_{\mathrm{A}}^{\times} \times\left(N(F) \backslash N\left(F_{\mathrm{A}}\right)\right)^{2}} K_{\Phi}\left(u_{1}, u_{2} z\right) \theta\left(u_{1}\right)^{-1} \theta\left(u_{2}\right) d u_{2} \chi \eta^{n / 2}(z) d z .
\end{aligned}
$$

Now we choose a set of representatives $\{\xi\}$ for the orbits of $F^{\times} \times G L(n, E)$ in $S(F)$, where $F^{\times}$operates by multiplication. For each $\xi$ we replace (11) by

$$
\int f^{\xi}(\tilde{h} g) \chi(\lambda(\tilde{h})) d \tilde{h}=\int \Psi\left[{ }^{t} \bar{g} \xi z g\right] \chi(z) d z .
$$

We now define a new kind of relative Bessel distributions

$$
\mathcal{M} \mathcal{R}_{\Pi}^{\xi}\left(f^{\xi}\right)=\sum \int \Pi\left(f^{\xi}\right) \phi_{i}(\tilde{h}) \chi(\lambda(\tilde{h})) d \tilde{h} \overline{\int \phi_{i}(u) \bar{\theta}(u \bar{u}) d u} .
$$

We now get instead of (19)

$$
\sum_{\xi} \sum_{\Pi} \mathcal{M} \mathcal{R}_{\Pi}^{\xi}\left(f^{\xi}\right)=\sum_{\pi} \mathcal{B}_{\pi}(\Phi)
$$

The end of the proof is the same as before.

Historical remarks. - For $n=2$, the study of the poles of the Asai L-function in [5] proves that the base change $\Pi$ of a representation $\pi$ with central character $\omega$ is distinguished by the character $\omega \eta \circ$ det of the group $G L\left(2, F_{\mathbb{A}}\right)$ and not distinguished by the character $\omega \circ \operatorname{det}$ of $G L\left(2, F_{\mathbb{A}}\right)$ (this fact is used explicitly in [6, (1), middle of p. 388]). Equivalently, the representation $\Pi$ is distinguished by the character $\omega \eta$ of the split unitary similitude group but not distinguished by the character $\omega$. We recall the proof. Let $\omega_{1}$ be an idele class character of $F$. Let $\Pi$ be a cuspidal automorphic representation of $G L\left(2, E_{\mathbb{A}}\right)$ with central character $\Omega$. Assume that the restriction of $\Omega$ to $F$ is $\omega_{1}^{2}$. Recall the Asai $L$-function $L\left(s, \Pi, \omega_{1}^{-1}\right.$, Asai). In this context, it is essentially defined by the condition that an integral of the form

$$
\int_{Z\left(F_{\mathrm{A}}\right)} \phi(g) E(g, \Phi, s) \omega_{1}^{-1}(\operatorname{det} g) d g
$$

is an entire multiple of the $L$ function. Here $\phi$ is in the space of $\Pi$ and $E(g, \Phi, s)$ is the Eisenstein series on $G L\left(n, F_{\mathrm{A}}\right)$ associated with a Schwartz-Bruhat function $\Phi$ on $F_{\mathbb{A}}^{n}$. In particular the (partial) Asai $L$-function has a pole at $s=1$ if and only if $\Pi$ is distinguished by $\left(G L\left(2, F_{\mathbb{A}}\right), \omega_{1}\right)$. Now if $\Pi$ is the base change of $\pi$ with central character $\omega$ then we have the following relations between (partial) $L$ functions:

$4^{\mathrm{e}}$ SÉRIE - TOME $38-2005-\mathrm{N}^{\circ} 4$ 


$$
\begin{aligned}
L\left(s, \Pi, \omega^{-1} \eta, \text { Asai }\right) & =L(s, \pi \times \tilde{\pi} \otimes \eta) \frac{L\left(s, 1_{F}\right)}{L(s, \eta)}, \\
L\left(s, \Pi, \omega^{-1}, \text { Asai }\right) & =L(s, \pi \times \tilde{\pi}) \frac{L(s, \eta)}{L\left(s, 1_{F}\right)}, \\
L(s, \Pi \times \widetilde{\Pi}) & =L(s, \pi \times \tilde{\pi}) L(s, \pi \times \tilde{\pi} \otimes \eta) .
\end{aligned}
$$

The function $L(s, \pi \times \tilde{\pi})$ has a pole at $s=1$ while the last relation shows that the function $L(s, \pi \times \tilde{\pi} \otimes \eta)$ does not have a pole at $s=1$ and does not vanish at $s=1$. The assertion follows.

The result of Proposition 7 is conjectured in $[8,(1)]$ in the context of $G L(n)$ and reproved by a relative trace formula as in the original work of Ye [18, (1)] in the context of $n=2$. At the time, the general conjecture with the exponent $n / 2$ was motivated by the results of [5] as referenced in $[6,(1)]$ in the case $n=2$ and by preliminary computations of Ye in the case $n=4$.

On the other hand, the author of [3] discusses distinction by the unitary group, first in the context of $G L(2)$. Earlier, he had proved the result of [5] that we recalled in the case $\omega=\eta$ but not in the case $\omega=1$. For the case $\omega=1$ he only discusses an example in [3]. ${ }^{2}$

It is natural to conjecture that one can choose the unitary group which distinguishes $\Pi$ to be quasi-split.

THEOREM 3. - Suppose each real place of F splits in E. Suppose $n$ is even. If $\Pi$ is a base change then $\Pi$ is distinguished by a quasi-split unitary group.

Proof. - The group $H^{\xi}$ is quasi-split if and only if at every inert place $v$, $\operatorname{det} \xi$ is equal to $\operatorname{det} w_{n}=(-1)^{n / 2}$ times a norm of $E_{w} / F_{v}$. We restrict the function $\Psi_{v}$ by demanding that it be supported on the set $S_{v}^{\prime}$ of elements whose determinant is $(-1)^{n / 2}$ times a norm. We take the function $\Phi_{v}$ to be supported on the set $G_{v}^{\prime}$ of elements $g$ such that $\operatorname{det} g$ is $(-1)^{n / 2}$ times a norm. As before, we introduce the function $\Phi^{\prime}$ defined by $\Phi^{\prime}[g]=\Phi\left[w_{n} g\right]$. We now get a spectral identity

$$
\sum_{\Pi} \mathcal{R}_{\Pi}^{\xi}\left(f_{\xi}\right)=\sum_{\pi} \mathcal{P}_{\pi}\left(\Phi^{\prime}\right)
$$

where

$$
\mathcal{P}_{\pi}\left(\Phi^{\prime}\right)=\sum \int \phi_{i}(u) \theta\left(u_{1}\right) d u_{1} \overline{\left.\int \phi_{(} u\right) \bar{\theta}\left(u_{2}\right) d u_{2}} .
$$

The function $\Phi_{v}^{\prime}$ is now supported on the group $G_{v}^{+}$of elements whose determinant is a norm. Moreover,

$$
\mathcal{P}_{\pi}\left(\Phi^{\prime}\right)=\mathcal{P}_{\pi \otimes \eta}\left(\Phi^{\prime}\right) .
$$

To conclude we have to see that the local Bessel distribution $\mathcal{P}_{\pi_{v}}$ has a non-zero restriction to $G_{v}^{+}$. This is checked in [6, (7)].

If the quadratic extension $E / F$ is arbitrary we have only a partial result.

THEOREM 4. - Suppose that $\Pi$ is an automorphic cuspidal representation of $G L\left(n, E_{\mathbb{A}}\right)$. Then $\Pi$ is distinguished by a unitary group if and only if it is a base change.

We proceed as before except that at any inert real place $v$ we choose the function $\Phi_{v}$ supported on the open set ${ }^{t} N_{v} A_{v} N_{v}$. Let $w$ be the place of $E$ above $v$. It is elementary that there is a

\footnotetext{
${ }^{2}$ In connection with this example the author makes a claim of priority. However, he does not acknowledge earlier references [5] and [6, (1)].
} 
function $\Psi_{v}$ supported on the open set of $H_{v}$ of matrices of the form

$$
{ }^{t} \bar{u} a u, \quad u \in N_{w}, a \in A_{v}
$$

which matches $\Phi_{v}$. Equating the discrete parts of the spectral expansions in the standard way, we get again:

$$
\sum_{\pi} \mathcal{B}_{\pi}(\Phi)=\sum_{\xi} \sum_{\Pi} \mathcal{R}_{\Pi}^{\xi}\left(f^{\xi}\right) .
$$

Fix a representation $\pi_{0}$ of $G L\left(n, F_{\mathbb{A}}\right)$ such that $\pi_{0} \otimes \eta_{E / F}$ is not equivalent to $\pi_{0}$. Thus the base change $\Pi_{0}$ of $\pi_{0}$ is automorphic cuspidal. Say $n$ is even. Then using the principle of linear independence of the characters of the Hecke algebra we get

$$
\mathcal{B}_{\pi_{0}}(\Phi)+\mathcal{B}_{\pi_{0} \otimes \eta_{E / F}}(\Phi)=\sum_{\xi} \mathcal{R}_{\Pi_{0}}^{\xi}\left(f^{\xi}\right)
$$

As before

$$
\mathcal{B}_{\pi_{0}}(\Phi)=\prod_{v} \mathcal{B}_{\pi_{0, v}}\left(\Phi_{v}\right), \quad \mathcal{B}_{\pi_{0} \otimes \eta_{E / F}}(\Phi)=\prod_{v} \mathcal{B}_{\pi_{0, v} \otimes \eta_{v}}\left(\Phi_{v}\right) .
$$

At a real place $v$ inert in $E$, by Lemma 23 we choose $\Phi_{v}$ so that

$$
\mathcal{B}_{\pi_{0, v}}\left(\Phi_{v}\right) \neq 0
$$

There is at least one finite place $u$ inert in $E$ such that

$$
\pi_{0, u} \nsucceq \pi_{0, u} \otimes \eta_{u} .
$$

We choose $\Phi_{u}$ so that

$$
\mathcal{B}_{\pi_{0, u}}\left(\Phi_{u}\right) \neq 0, \quad \mathcal{B}_{\pi_{0, u} \otimes \eta_{u}}\left(\Phi_{u}\right)=0
$$

Then we find

$$
\mathcal{B}_{\pi_{0}}(\Phi)=\sum_{\xi} \mathcal{R}_{\Pi_{0}}^{\xi}\left(f^{\xi}\right)
$$

We conclude that $\mathcal{R}_{\Pi_{0}}^{\xi}\left(f^{\xi}\right)$ is non-zero for at least one $\xi$ and the theorem follows. If $n$ is odd the proof is similar but simpler.

\section{REFERENCES}

[1] Arthur J., Clozel L., Simple Algebras, Base Change, and the Advanced Theory of the Trace Formula, Ann. of Math. Stud., vol. 120, Princeton University Press, Princeton, NJ, 1989.

[2] Casselman W., Shalika J., The unramified principal series of $p$-adic groups. II. The Whittaker function, Compositio Math. 41 (1980) 207-231.

[3] FLICKER Y., On distinguished representations, J. reine angew. Math. 418 (1991) 139-172.

[4] Goodman R., WALlaCh N., Representations and Invariants of the Classical Groups, Encyclopedia of Mathematics and its Applications, Cambridge University Press, Cambridge, 1998.

[5] Harder G., Langlands R., Rapoport M., Algebraische Zyklen auf Hilbert-BlumenthalFlächen, J. reine angew. Math. 366 (1986) 53-120.

$4^{\text {e }}$ SÉRIE - TOME $38-2005-\mathrm{N}^{\circ} 4$ 
[6] (1) JACQUET H., Sur un résultat de Waldspurger II, Compositio Math. 63 (1987) 315-389;

(2) JACQUET H., The continuous spectrum of the relative trace formula for $G L(3)$ over a quadratic extension, Israel J. Math. 89 (1995) 1-59;

(3) JACQUET H., A theorem of density for Kloosterman integrals, Asian J. Math. 2 (1998) 759778 ;

(4) JACQuet H., Transfert lisse d'intégrales de Kloosterman, C. R. Acad. Sci. Paris 335 (2002) 229-302;

(5) JACQUET H., Smooth transfer of Kloosterman integrals, Duke Math. J. 120 (2003) 121-152;

(6) JACQUET H., Facteurs de transfert pour les intégrales de Kloosterman, C. R. Acad. Sci. Paris. Ser. I 336 (2003) 121-124;

(7) JACQUET H., Factorization of period integrals, J. Number Theory 87 (1) (2001) 109-143;

(8) JACQUET H., Kloosterman Identities over a quadratic extension, Ann. of Math., submitted for publication.

[7] JACQUET H., RALlis S., Kloosterman integrals for skew symmetric matrices, Pacific J. Math. 154 (2) (1992) 265-283.

[8] (1) JACQUET H., YE Y., Une remarque sur le changement de base quadratique, C. R. Acad. Sci. Paris Sér. I Math. 311 (1990) 671-676;

(2) JACQUet H., Ye Y., Relative Kloosterman integrals for $G L(3)$, Bull. Soc. Math. France 120 (1992) 449-558;

(3) JACQUeT H., Ye Y., Distinguished representations and quadratic base change for $G L(3)$, Trans. Amer. Math. Soc. 348 (1996) 913-939;

(4) JACQUeT H., YE Y., Germs of Kloosterman integrals for GL(3), Trans. Amer. Math. Soc. 351 (1999) 1227-1255.

[9] KotTwitz R., Base change for unit elements of Hecke algebras, Compositio Math. 60 (1986) 237 250.

[10] LAPID E., On the fine spectral expansion of Jacquet's relative trace formula, Preprint.

[11] Lapid E., Rogawski J., Periods of Eisenstein series: the Galois case, Duke Math. J. 120 (2003) 153-226.

[12] LAPID E., RogawsKi J., Stabilization of periods of integrals of Eisenstein series and Bessel distributions on $G L(3)$ relative to $U(3)$, Doc. Math. 5 (2000) 317-350.

[13] Macdonald I.G., Symmetric Functions and Orthogonal Polynomials, Amer. Math. Soc. Univ. Lecture Ser., vol. 12, 1991.

[14] Mao Z., RALlis S., Howe duality and the trace formula, Pacific J. Math. 191 (2) (1999) 329-346.

[15] (1) NGô B.C., Le lemme fondamental de Jacquet et Ye en caractéristique positive, Duke Math. J. 96 (1999) 473-520;

(2) NGÔ B.C., Faisceaux pervers, homomorphisme de changement de base et lemme fondamental de Jacquet et Ye, Ann. Sci. École Norm. Sup. 32 (1999) 619-679.

[16] Shalika J., The multiplicity one theorem for $G L_{n}$, Ann. of Math. 100 (2) (1974) 171-193.

[17] Shintani T., On an explicit formula for class-1 Whittaker functions on $G L_{n}$ over $P$-adic fields, Proc. Japan Acad. 52 (1976) 180-182.

[18] (1) YE Y., Kloosterman integrals and base change for $g l(2)$, J. reine angew. Math. 400 (1989) 57121 ;

(2) YE Y., The fundamental lemma of a relative trace formula for GL(3), Compositio Math. 89 (1993) 121-162;

(3) YE Y., An integral transform and its applications, Math. Ann. 30 (1994) 405-417.

(Manuscrit reçu le 22 décembre 2004; accepté, après révision, le 15 avril 2005.)

\footnotetext{
Hervé JACQUET

Mathematics Department MC 4408,

Columbia University,

New York, NY 10027, USA

E-mail: hj@math.columbia.edu
} 